\title{
Improving Signal to Noise Ratio in Ultra High Field Magnetic Resonance Imaging
}

\author{
A DISSERTATION \\ SUBMITTED TO THE FACULTY OF THE \\ UNIVERSITY OF MINNESOTA \\ BY
}

Nader Tavaf

IN PARTIAL FULFILLMENT OF THE REQUIREMENTS

FOR THE DEGREE OF

DOCTOR OF PHILOSOPHY

Advised by: Pierre-François Van de Moortele, MD, $\mathrm{PhD}$

March 2021 
CNader Tavaf 2021 
I would like to sincerely thank my advisor, Pierre-François Van de Moortele, for his technical advice and direction as well as his personal support. I thank Pierre for the opportunity he gave me, for the trust he placed in me, for the $12+$ hour long, $11 \mathrm{pm}$ experiments at 10.5T, for sharing my excitement for hands-on work, and for the independence he gave me in setting the pace of my efforts. I am also deeply grateful to Gregor Adriany and Kamil Ugurbil for sharing their expertise and for the key role they had in steering my research. I thank Pierre, Gregor and Kamil for being incredibly supportive and generous with their time.

This work would not have been possible without substantive contributions of many researchers, including, in no particular order, Russell L. Lagore, Steve Jungst, Andrea Grant, Jerahmie Radder, Lance DelaBarre, Edward Auerbach, Gregor Adriany, Pierre-François Van de Moortele, and Kamil Ugurbil. I appreciate contributions from Yigitcan Eryaman, Myung Kyun Woo, Steen Moeller, Shajan Gunamony, and Matt Waks.

Thank you to members of my committee, Patrick Bolan, Greg Metzger, Gregor Adriany, and Pierre-François Van de Moortele, for discussions, constructive feedback, and directions of research. Special thanks to Kamil Ugurbil and Gregor Adriany for providing inspiration, perspective and context for this research as well as continuous guidance and technical direction during the development of this work. I am grateful to Victor Barocas and Rachel Jorgenson for their unparalleled proactive mentoring and passionate support of biomedical engineering graduate students.

I would like to express my thanks for friendships. Thank you Russell for candid expressions of reality without sugar-coating, Gregor for optimism and understanding, Andrea for unfailing respect for colleagues and perseverance in wrestling with the 10.5T, Steve 
for non-egotistic, non-judgmental presence, Jerahmie for inclusion, and Kamil for fatherly care. I also thank Josh Punnoose for listening compassionately. Thanks to Amirsina Torfi for pushing me to apply to graduate school. Thanks to Mehran and Hoda Mousavi for keeping us company through the years, irrespective of our idiosyncrasies. I am grateful to Abbas Sohrabpour for significant help and advice. Thanks to my labmates, especially Sean Moen for encouraging me to have fun, Kellen Mulford for help, and Ruoyun Emily Ma for occasionally refusing to help so I learn to be independent. Thanks to Greg Metzger for introducing Pierre-François to me. Thanks to Rachel Jorgenson and Victor Barocas for I would not have made it to the US without their unequivocal support. Thanks to Nicole Wilkinson for administrative help.

I thank all of the members of the Center for Magnetic Resonance Research (CMRR) family for creating an admirably diverse, inclusive, and collaborative culture. I take pride in having been part of this unique family.

Finally, thanks to my wife, Maryam, for stepping with me through darkness and for being the pillar on which I lean. No words can capture my gratitude for my family, my parents and my in-laws. I'm deeply affected by the sacrifices they made to get me here.

The research presented here received financial support from National Institutes of Health (NIH) U01 EB025144, P41 EB027061, S10 RR029672, P30 NS076408 and R34 AG055178. 


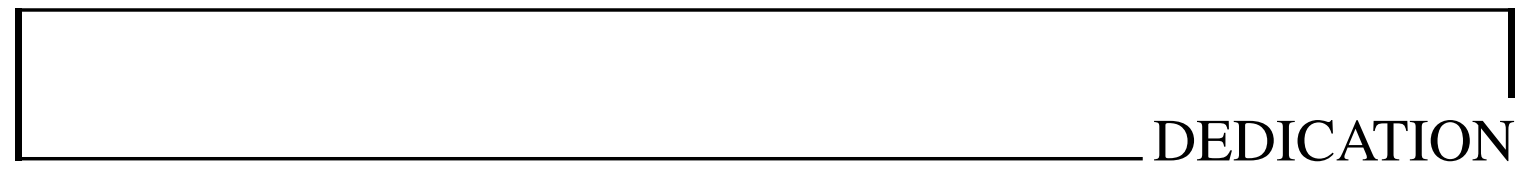

To the loved ones we lost on the way. 
Ultra-High Field (UHF) Magnetic Resonance Imaging (MRI) advantages, including higher image resolution, reduced acquisition time via parallel imaging, and better Signal-to-Noise Ratio (SNR) have opened new opportunities for various clinical and research projects, including Functional Magnetic Resonance Imaging (fMRI), brain connectivity mapping, and anatomical imaging. The advancement of these UHF MRI performance metrics, especially SNR, was the primary motivation of this thesis.

Unaccelerated SNR depends on receive array sensitivity profile, receiver noise correlation and static magnetic field strength. Various receive array decoupling technologies, including overlap/inductive and preamplifier decoupling, were previously utilized to mitigate noise correlation. In this dissertation, I developed a novel self-decoupling principle to isolate elements of a loop-based receive array and demonstrated, via full-wave electromagnetic/circuit co-simulations validated by bench measurements, that the selfdecoupling technique provides inter-element isolation on par with overlap decoupling while self-decoupling improves SNR. I then designed and constructed the first self-decoupled 32 and 64 channel receiver arrays for human brain MRI imaging at 10.5T / 447MHz. Experimental comparisons of these receive arrays with the industry's gold-standard 7T 32 channel receiver resulted in 1.81 times and 3.53 times more average SNR using the 10.5T 32 and 64 channel receivers I built, respectively.

To further improve the SNR of accelerated MRI images, I developed a novel data-driven model using a customized conditional Generative Adversarial Network (GAN) architecture for parallel MRI image reconstruction and demonstrated that, when applied to human brain images subsampled with rate of 4, the GAN model results in a Peak Signal-to-Noise Ratio (PSNR) of 37.65 compared to GeneRalized Autocalibrating Partial Parallel Acquisition 


\section{(GRAPPA)'s PSNR of 33.88.}

In summary, the work presented in this dissertation improved the SNR available for human brain imaging and provided the experimental realization of the advantages anticipated at 10.5T MRI. The insights from this thesis inform future efforts to build self-decoupled transmit arrays and high density (i.e. 128 channel) loop-based receive arrays for human brain MRI especially at ultra-high field as well as future studies to utilize deep learning techniques for reconstruction and post-processing of parallel MRI images. 
Acknowledgements $\quad$ i

Dedication $\quad$ iii

Abstract $\quad$ iv

Table of Contents vi

List of Tables $\quad$ ix

List of Figures $\quad$ x

List of Abbreviations $\quad$ xi

Preface $\quad$ xiii

1 Fundamentals 1

1.1 MRI Physics and Ultra-High Field . . . . . . . . . . . . . . . . 2

1.1 .1 The MRI Signal . . . . . . . . . . . . . . 2

$1.1 .2 \quad k$-space .......................... 4

1.2 RF Coils in MRI . . . . . . . . . . . . . . . . . 5

1.2.1 Receive Arrays . . . . . . . . . . . . . . 5

1.2.2 RF Arrays and SNR . . . . . . . . . . . . . . 5

1.3 Parallel Imaging and Reconstruction . . . . . . . . . . . . . . . . . 7

1.3.1 GRAPPA and $g$-Factor . . . . . . . . . . . . 8

1.3.2 RF Arrays and PI $g$-factor . . . . . . . . . . . . . . . . . 10

1.4 Deep Learning for MRI Reconstruction . . . . . . . . . . . . . . . . . . . . . 11

1.4.1 Generative Adversarial Networks . . . . . . . . . . . . 11 
2 Self-Decoupling of Receive Arrays 14

2.1 Introduction . . . . . . . . . . . . . . . . 15

2.2 Methods . . . . . . . . . . . . . . . . . . . . . . . . . . . . . . .

2.3 Results . . . . . . . . . . . . . . . . . . 20

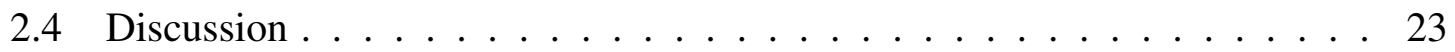

3 32-Channel Receive Array at 447MHz 25

3.1 Introduction . . . . . . . . . . . . . . 25

3.2 Methods . . . . . . . . . . . . . . . . . . 27

3.2.1 The transmitter . . . . . . . . . . . . . . . 27

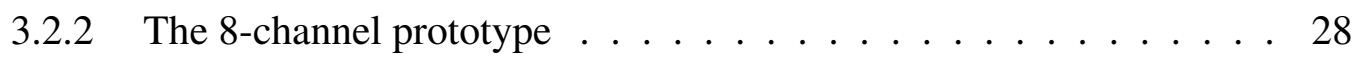

3.2.3 The 32 -channel receiver . . . . . . . . . . . . . . . . . 29

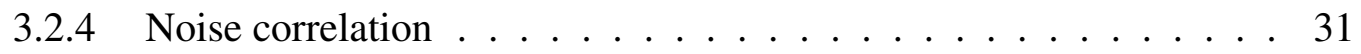

3.2.5 Transmit-receive interaction .................... 33

3.2 .6 SNR and $g$-factor . . . . . . . . . . . . . . . . . . 34

3.3 Results . . . . . . . . . . . . . . . . . 36

3.3.1 Receiver noise correlation and $\mathrm{Tx} / \mathrm{Rx}$ interaction . . . . . . . 36

3.3.2 SNR and $g$-factor . . . . . . . . . . . . . . . 37

3.4 Discussion ............................ 41

4 64-Channel Receive Array at 447MHz 45

4.1 Introduction . . . . . . . . . . . . . . . . . . 45

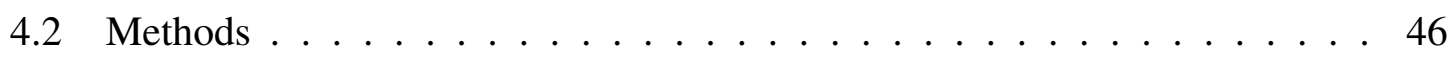

4.3 Results . . . . . . . . . . . . . . . . . . . . . . . . . . . . . . . . . . 48

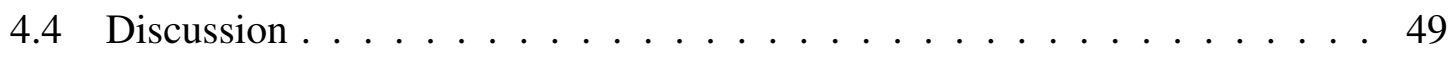

5 GANs for Parallel Image Reconstruction 53

5.1 Introduction . . . . . . . . . . . . . . . 53

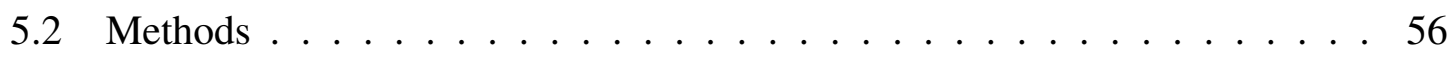

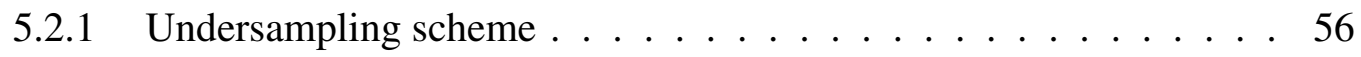

5.2 .2 Reconstruction method . . . . . . . . . . . . . . 57

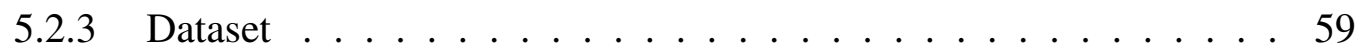

5.2 .4 Evaluation metrics .......................... 60

5.2.5 Training and implementation details . . . . . . . . . . . . 60

5.3 Results . . . . . . . . . . . . . . . . 61

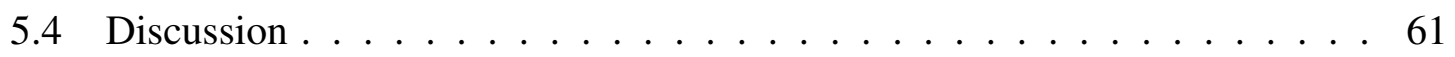

6 Conclusions and Future Directions 66

6.1 Self-Decoupling . . . . . . . . . . . . . . . 66

6.232 -Channel Receiver . . . . . . . . . . . . . . . . . . 67

6.3 64-Channel Receiver . . . . . . . . . . . . . . . . . . 67

6.4 GANs for PI Reconstruction . . . . . . . . . . . . . . . . . 68

6.5 Future Directions . . . . . . . . . . . . . . . 68 
Bibliography 


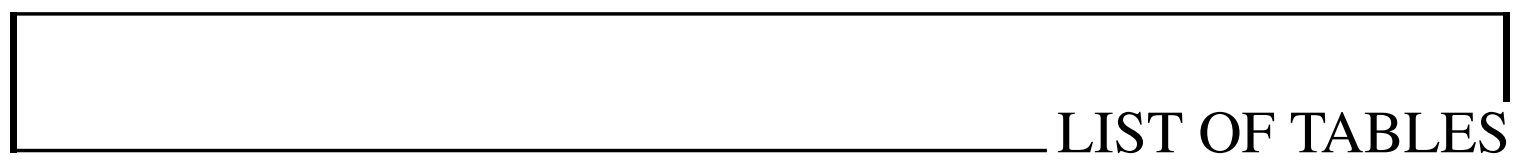

2.1 On-the-bench vs. simulated component values . . . . . . . . . . . . . . . 19

2.2 SNR gain of self-decoupling vs overlap . . . . . . . . . . . . 22

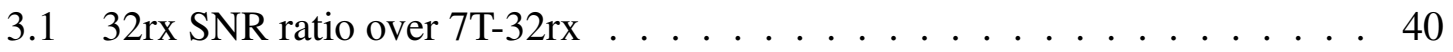

$3.232 \mathrm{rx}$ g-factor comparison ...................... 41

$4.164 \mathrm{rx}$ SNR ratio over $32 \mathrm{rx} \ldots \ldots \ldots \ldots \ldots$

5.1 Performance of GAN vs GRAPPA . . . . . . . . . . . . . . 61 
1.1 The concept of GRAPPA . . . . . . . . . . . . . . . 9

2.1 Schematic of feed circuit . . . . . . . . . . . . . . . . . . 19

2.2 Self-decoupling S-parameters . . . . . . . . . . . . . . . 21

2.3 Self-decoupling field and current density . . . . . . . . . . . . . . . 21

2.4 Self-decoupling vs overlapped RSOS field maps . . . . . . . . . . . . 23

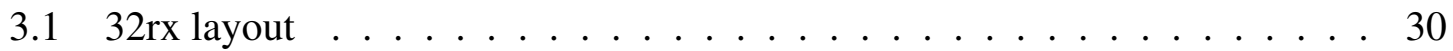

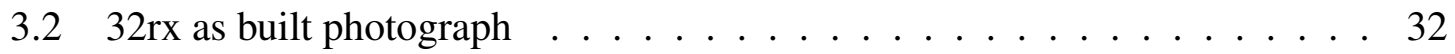

$3.332 \mathrm{rx}$ noise correlation . . . . . . . . . . . . . 37

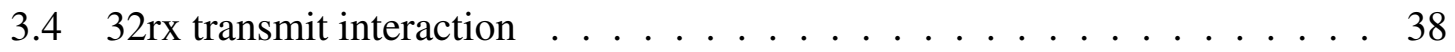

$3.532 \mathrm{rx}$ axial SNR comparison with 7T-32rx . . . . . . . . . . 38

$3.632 \mathrm{rx}$ 3D SNR comparison with 7T-32rx ................ 39

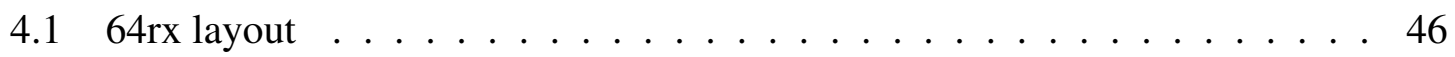

4.264 rx as-built photograph . . . . . . . . . . . . . . . 48

4.364 rx noise correlation . . . . . . . . . . . . . . . . . . . 49

$4.464 \mathrm{rx}$ axial SNR comparison with $32 \mathrm{rx} \ldots \ldots \ldots \ldots$. . . . . . . . 50

$4.564 \mathrm{rx}$ 3D SNR comparison with $32 \mathrm{rx} \ldots \ldots \ldots \ldots$

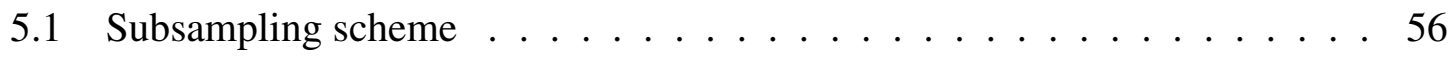

5.2 Symbolic network architecture . . . . . . . . . . . . 58

5.3 Performance comparison at $\mathrm{R}=4 \ldots \ldots \ldots$. . . . . . . . . . . . . . . . . . . . . . . . 62

5.4 Performance comparison at $\mathrm{R}=8 \ldots \ldots \ldots \ldots$

5.5 Denoising and artifact suppression with GANs . . . . . . . . . . . . 65 
ACS Auto Calibration Signal

AFI Actual Flip Angle Imaging

AWG American Wire Gauge

CMRR Center for Magnetic Resonance Research

EM ElectroMagnetic

EPI Echo Planar Imaging

FDA Food and Drug Administration

fMRI Functional Magnetic Resonance Imaging

GAN Generative Adversarial Network

GRAPPA GeneRalized Autocalibrating Partial Parallel Acquisition

GRE Gradient Echo

ICEAA International Conference on Electromagnetics in Advanced Applications

IDE Investigational Device Exemption

IEEE Institute of Electrical and Electronics Engineers

ISMRM International Society for Magnetic Resonance in Medicine

MRI Magnetic Resonance Imaging

MRM Magnetic Resonance in Medicine

NIH National Institutes of Health

NMR Nuclear Magnetic Resonance

PI Parallel Imaging

PSNR Peak Signal-to-Noise Ratio

pTx Parallel Transmit

RF Radio Frequency 
ROI Region of Interest

RSOS Root Sum of Squares

SAR Specific Absorption Rate

SENSE SENSitivity Encoding

SMASH SiMultaneous Acquisition of Spatial Harmonics

SNR Signal-to-Noise Ratio

SOM Sum of Magnitude

SSIM Structural Similarity

UHF Ultra-High Field 
MRI is a non-invasive medical imaging modality with extensive clinical and research applications across disciplines. Such applications give impetus to the fundamental research effort which is the focus of this thesis: improving the SNR of MRI imaging of the human brain.

\section{THE PURSUIT OF SNR}

There is a fundamental trade-off between the per voxel SNR and image resolution. Clinical and research applications continuously require higher resolutions to discern smaller anatomical structures. Given the SNR versus resolution trade-off, these applications send researchers on a mission to find ways to compensate for the SNR lost due to higher resolution. Since a primary avenue is to start from higher SNR (independent of particular imaging parameters) by increasing the static magnetic field strength, there has been a push for ultra-high field MRI.

Beyond field strength, Radio Frequency (RF) coils are a principal determinant of intrinsic SNR as the MRI signal intensity is proportional to the sensitivity profile of the particular RF coil employed in the experiment. As it relates to RF receivers, the available SNR can be increased by designing and building receiver coils with either increased sensitivity and/or reduced noise. Furthermore, arrays of receiver elements provide the opportunity to en- 
hance the available SNR by combining the signal from ideally-independent coil elements. This opens the discussion to a host of reconstruction techniques which exploit, each in its own way, the information redundancy of such receive arrays. As MRI acquisitions can be time-consuming especially at higher resolutions, the need to reduce the acquisition time (in order to increase patient throughput, mitigate motion artifacts, etc) brings the additional receiver array information into the spotlight. This extra information allows for improved reconstruction of MRI images from undersampled signals, effectively reducing the scan acquisition time. The undersampling, however, comes at the expense of amplified noise.

The pursuit of SNR via better RF coils was the initial and primary focus of my research. I started by working on a novel self-decoupling technique to mitigate the noise correlation of loop-based receive arrays and improve their sensitivity. When I started my research at the RF lab of the CMRR, the maximum noise correlation of CMRR's 7T 64-channel receive array stood at 0.67 [1]. As of this writing, I have built a 10.5T 64-channel receiver with a max noise correlation of 0.31 [2]. The self-decoupling technique paved the way for the next step: building high density receiver arrays with effective inter-element isolation. I designed and constructed a 32 and a 64 channel receive array for human brain imaging at $10.5 \mathrm{~T}$ and demonstrated, EXPERIMENTALLY, more than three fold SNR enhancement compared to prior 7T industry standards $[2,3]$. To further improve the SNR of images acquired using these receive arrays, I continued by introducing a novel data driven reconstruction technique for undersampled MRI images which addresses the noise amplification issue of conventional reconstruction methods.

\section{OUTLINE OF THE THESIS}

The purpose of this thesis is to realize the potential SNR improvements promised by ultrahigh field MRI. This purpose is pursued in two contiguous fronts: building better receive arrays and improving reconstruction techniques.

Chapter 1 starts by laying out the fundamental concepts and establishes the terminology required to discuss the works presented here. MRI physics are briefly reviewed. SNR advantages of ultra-high field MRI and the role of RF antennas in realizing these advantages are discussed. Principles of image reconstruction using multi-channel receive arrays and conventional parallel imaging techniques are reviewed to lay the groundwork for the deep learning methods of MRI reconstruction which follow.

Chapter 2 presents the novel self-decoupling technique and results, demonstrating ef- 
fective interelement isolation and SNR improvements compared to overlapped decoupling. Self-decoupling paves the way for Chapter 3 and Chapter 4, where high density receive arrays and their performance characterizations (on the bench and in the MRI scanner) are presented. Chapter 4 wraps up the hardware effort with a discussion of the SNR gains using the high density receive arrays at ultra-high field. Chapter 5 delves into the reconstruction techniques of undersampled image acquisitions and presents a novel method to mitigate noise amplification and improve the fidelity of the reconstructed images. Chapter 6 summarizes the conclusions of the thesis and ends with a discussion on promising future directions.

These chapters often borrow from what was already published and/or presented in:

- N. Tavaf, R. L. Lagore, S. Moen, et al., "A 15-channel loop dipole array for in-vivo swine head MR imaging at 10.5T," ISMRM, 2019, p. 1445.

- G. Adriany, J. Radder, N. Tavaf, et al., "Evaluation of a 16-Channel Transmitter for Head Imaging at 10.5T," ICEAA, IEEE, Sep. 2019, pp. 1171-1174.

- N. Tavaf, R. L. Lagore, S. Jungst, et al., "Developing High Channel Count Receive Arrays for Human Brain Imaging at 10.5T," ISMRM, 2020, p. 1961.

- N. Tavaf, R. L. Lagore, S. Jungst, et al., "A Self-Decoupled 32 Channel Receive Array for Human Brain Magnetic Resonance Imaging at 10.5T,” MRM, accepted Mar 2021.

- M. K. Woo, L. Dela Barre, M. Waks, et al., "A Novel High Density 32-channel Sleeve Antenna Receiver Array for the Human Head Imaging at 10.5 T," ISMRM, 2021, p. 236.

- N. Tavaf, S. Jungst, R. L. Lagore, et al., "A Self-decoupled 64 Channel Receive Array for Human Brain MRI at 10.5T,” ISMRM, 2021, p. 2618.

- N. Tavaf, A. Torfi, K. Ugurbil, et al., "GRAPPA-GANs for Parallel MRI Reconstruction”, arXiv:2101.03135, Jan. 2021

- N. Tavaf, J. Radder, R. Lagore, et al., "Design of a Self Decoupled 16 Channel Transmitter for Human Brain MRI at 447 MHz”, arXiv:2103.07516, Mar 2021.

I have tried to keep these chapters to-the-point and limit reiterations of what was already extensively discussed in the works of other researchers. While Dr. Pierre-François Van de Moortele, whose knowledge of MRI seems inexhaustible, has reviewed this thesis for conspicuous mistakes, he is not responsible for the conclusions I have drawn or any errors that remain. Should a reader be enthusiastic enough to try to replicate some of the works reported here, there will most certainly be opportunities to suggest improvements or revisions. However, that reader may sympathize with Tai T'ung's words: "were I to await perfection, my book would never be finished" [4]. 
CHAPTER 1

FUNDAMENTALS

It's up to you.

Pierre-François - Jan 2018.

MRI has turned into an indispensable part of routine clinical examinations and biomedical research efforts. Improvements in the resolution and SNR made possible at UHF MRI and reductions in scan acquisition time have promoted MRI to preeminence in various applications [5-8 $]^{1}$. MRI applications that rely on the latter advantages include fMRI studies [6, 8-11], brain connectivity mapping using diffusion-weighted MRI [12-15] and anatomical imaging [16-19]. Such applications depend on ultra-high field advantages made possible by the contributions of interconnected components of the imaging pipeline, including better radio-frequency coils and reconstruction algorithms. To discuss these contributing components, a brief review of MRI physics is necessary.

\footnotetext{
${ }^{1}$ The references in this paragraph are provided as examples. It is beyond the scope of this chapter to list all of the relevant literature and applications that benefit from ultra-high field MR advantages. More generally, the focus of this chapter is to introduce the fundamental concepts, not to provide an exhaustive literature review in each section. A more inclusive review of relevant prior art is provided in the introduction of subsequent chapters.
} 


\subsection{MRI Physics AND UltRA-High FIELD}

With magnetization, $\vec{M}$, representing the average magnetic dipole moment density of the sample, it can be shown that in equilibrium, $\vec{M}=M_{0} \hat{z}$, where $M_{0}$ is the initial magnitude of the magnetization aligned with $\hat{z}$ the identity vector in the direction of the external static magnetic field, $B_{0}$ [20]. If a RF pulse (defined as a magnetic field, $B_{1}$, applied on-resonance for a finite time duration) disturbs the equilibrium, the magnetization's subsequent trajectory follows the Bloch equation [20]:

$$
\frac{d \vec{M}}{d t}=\gamma \vec{M} \times \vec{B}_{e x t}+\frac{1}{T_{1}}\left(M_{0}-M_{z}\right) \hat{z}-\frac{1}{T_{2}} \vec{M}_{\perp}
$$

where $\gamma$ is the gyromagnetic ratio, $\vec{B}_{\text {ext }}$ the external magnetic field, $T_{1}$ the longitudinal relaxation constant, $T_{2}$ the transverse decay constant, and $\vec{M}_{\perp}$ is the transverse component of the magnetization perpendicular to $\vec{M}_{z}=M_{z} \hat{z}$. The transient and steady state solutions to the Bloch equation are known, both for the case when $\vec{B}_{\text {ext }}$ includes only the static magnetic field and for the transmit case when the RF pulse is on.

\subsubsection{The MRI Signal}

After the RF pulse is turned off, the solutions to Eq. 1.1 provide the trajectory of the magnetization over time and space $\vec{M}(\vec{r}, t)$. The magnetization's precession generates a timevariant magnetic field $\vec{B}(\vec{r}, t)$ which induces an electromotive force (emf $=-\partial \Phi / \partial t)$ in a resonant closed circuit (i.e. a loop coil) placed inside the field as the magnetic flux $\Phi$ through the coil changes over time, according to Faraday's law. The current induced in the loop in turn gives rise to a magnetic field, $\vec{B}_{1}^{-}$, which can be evaluated at the sample position. Due to the principle of reciprocity, the flux through the detection coil due to the magnetization can be found by calculating the flux that would emanate from the detection 
coil, per unit current, through the (spatio-temporally varying) magnetization. Therefore, the electromotive force induced in the coil can be expressed as [20]:

$$
s=-\frac{\partial}{\partial t} \int_{\text {sample }} \vec{M}(\vec{r}, t) \cdot \vec{B}_{1}^{-}(\vec{r}) d^{3} r
$$

Eq. 1.2 is the MRI signal, $s$, in its general form. The signal dependence on spin density, $\rho$, and transmit RF pulse, $B_{1}^{+}$, is implied in the magnetization term, $\vec{M}(\vec{r}, t)$. Solutions to Eq. 1.1 and the assumptions of uniform static magnetic field, $B_{0}$, and negligible relaxation and decay during short transmit pulses (i.e. $\gamma B_{0} \gg \max \left\{1 / T_{1}, 1 / T_{2}\right\}$ ) are used to solve Eq. 1.2 for specific signal acquisition sequences, such as gradient echo and spin echo. In the general case, as $M_{0} \propto B_{0}$ and the frequency $\omega_{0}=\gamma B_{0}$, it follows from Eq. 1.2 that the signal magnitude, $s$, is proportional to [20]:

$$
s \propto B_{0}^{2} \cdot B_{1}^{-}(\vec{r})
$$

where $B_{1}^{-}(\vec{r})$ is the magnetic field of the receive coil at location $\vec{r}$ of the sample. Eq. 1.3 motivates the push towards using UHF ( $B_{0} \geq 7 \mathrm{~T}$ ) MRI systems as well as building high sensitivity RF receive coils ${ }^{2}$.

Eq. 1.3 underestimates the complexity of the challenge. In the more general SNR equation, noise is also frequency-dependent. If one describes SNR as $\mathrm{SNR} \propto B_{0}^{x}$, the exponent $x$ has been and continues to be a subject of research and debate. At ultra-high field, with increased frequency, the complexity of the spatial distribution of electromagnetic fields increases, and the SNR gains based on the exponent $x$ are not homogeneous through space. Analytical solutions, simulations and experiments have reported various numbers for $x$

\footnotetext{
${ }^{2}$ On a historical note, MRI started from much lower field strengths. Mansfield's in-vivo images (the cross section of a human finger) were at $15 \mathrm{MHz}(0.35 \mathrm{~T})$ [21]. Nuclear Magnetic Resonance (NMR) images by Lauterbur (dubbed zeugmatography) were at $60 \mathrm{MHz}(1.4 \mathrm{~T})$ [22]. The evolution to higher field strengths and frequencies is more recent and is motivated in large part by the higher resolution required for brain neuroscience applications. See [7] for a detailed history of ultra-high field MRI
} 
(averaged over the sample), mostly in the range of 1 to 2 . For a widely cited study, see Pohmann et al. [23] which reports $x \simeq 1.65$ on average. Irrespective of the exact value of $x$ achieved in practice, the verdict of Eq. 1.3 is motivating.

\subsection{2 $k$-space}

Imaging refers to determination of the spin density distribution over space, $\rho(\vec{r})$. Since nuclei precess at different frequencies where the external magnetic field is slightly different, the local spatial distribution of spins can be determined from the frequency content of the MRI signal, provided a well-defined spatial field variation is superimposed on the homogeneous static field ${ }^{3}$.

Noting that $M_{\perp}(\vec{r}, t) \propto \rho(\vec{r})$, assuming uniform transmit and receive fields, negligible relaxation effects, and no inherent offset precession frequencies ${ }^{4}$, it can be shown that Eq. 1.2 results in [20]:

$$
\begin{aligned}
& s(\vec{k})=\int \rho(\vec{r}) e^{-i \vec{k} \cdot \vec{r}} d^{3} r=\mathcal{F}[\rho(\vec{r})] \\
& \rho(\vec{r})=\int s(\vec{k}) e^{+i \vec{k} \cdot \vec{r}} d^{3} k=\mathcal{F}^{-1}[s(\vec{k})]
\end{aligned}
$$

where $s(\vec{k})$ is signal measured at the scanner, the $k$-space is defined as $\vec{k}(t)=\gamma \int \vec{G}(t) d t$ with $\vec{G}(t)$ being the gradient field and $\mathcal{F}$ the Fourier transform ${ }^{5}$. Eq. 1.4 is the fundamental Fourier pair forming three dimensional MRI images.

\footnotetext{
${ }^{3} G_{z}=10 \mathrm{mT} / \mathrm{m}$ is indicative of the order of gradients while $\delta=10 \mathrm{ppm}$ is indicative of the order of field inhomogeneities. Field inhomogeneity is at most a few percentages of maximum gradient, so static field can be taken as uniform.

${ }^{4}$ The primary reason that frequency is space/time dependent is presence of gradient fields.

${ }^{5}$ The $k$-space representation is often attributed to [24]. Gradients are magnetic fields with known (ideally linear) spatial variation superimposed on $B_{0}$ to provide spatial frequency encoding.
} 


\subsection{RF COILS IN MRI}

The term $R F$ coil refers to certain resonant electronic hardware components of the MRI system used to transmit or receive (sense) magnetic fields. Receivers are a subset of RF coils used as sensors to pick up the MRI signal (Eq. 1.2). The $B_{1}^{-}$in Eq. 1.2 is the magnetic field profile of the receiver. While there are various types of receivers, the focus of the following chapters is on arrays of receive elements, with each element being a resonant loop. A single loop is an LC circuit resonant at the frequency of $f=1 /(2 \pi \sqrt{L C})$, where $L$ is inductance (in nano Henries, $n H$ ) and $C$ is capacitance (in pico Farads, $p F$ ).

\subsubsection{Receive Arrays}

In their seminal work, Roemer et al. [25] demonstrated that sensing the MRI signal simultaneously with an array of loops can outperform a single, large loop covering the same area. Roemer presented a method for optimally combining the signal from the receive elements of the array given their sensitivity profile and noise correlation information. Roemer's demonstration of improved sensitivity of a surface array compared to a single loop provided the basis of the subsequent interest and focus on receive arrays in the MRI community. A second advantage of receive arrays is the possibility to use their extra information to reconstruct undersampled $k$-space signals and accelerate image acquisition, details of which are covered in Section 1.3. Later studies demonstrated that increased channel count of conformal receive arrays leads to better performance in terms of sensitivity and acceleration potential especially at ultra-high fields (e.g. [26-32]).

\subsubsection{RF Arrays and SNR}

As the magnetic field profile of each coil element, $B_{1, i}^{-}(\vec{r})$, is a function of pixel location, for a uniform sample, with $\mathcal{B}$ being a $1 \times N_{c h}$ vector composed of the $B_{1, i}^{-}(\vec{r})$ for $i \in\left(1, N_{c h}\right)$ 
individual coils, assuming the noise covariance matrix (i.e. the noise covariance between elements of the receive array), $\Psi$, is proportional to the identity matrix ${ }^{6}$, then:

$$
\operatorname{RSOS}=\sqrt{\mathcal{B}^{H} \mathcal{B}}
$$

is the Root Sum of Squares (RSOS) reconstruction at the image domain with $H$ being the Hermitian transpose. If the noise covariance matrix is measured (in the absence of excitation), the covariance-weighted root-sum-of-squares combination of the signal results in the signal-to-noise ratio of the array:

$$
\mathrm{SNR}=\sqrt{\mathcal{B}^{H} \Psi^{-1} \mathcal{B}}
$$

To explain the SNR gain as a function of number of channels, consider an array of $N_{c h}$ identical small loops covering the surface of an sphere. For the ideal case with the noise correlation being equal to an identity matrix (i.e. equal levels of noise in all channels and zero inter-element noise correlation), Eq. 1.5 results in an $\sqrt{N_{c h}}$ improvement at the center of the sphere (simple averaging if all channels have identical $B_{1}^{-}$profiles and the voxel of interest is equidistant from all loops). This means that, comparing an 32-channel receive array with an 64-channel receive array, assuming both arrays employ identical loop elements ${ }^{7}$, one should observe $\sqrt{64 / 32}=1.41$ times increase in average RSOS at the central voxel using the 64-channel array. Eq. 1.6 is, however, a reminder that, in practice, noise correlation affects the gains realized at higher channel counts. It is, at least in part, the fundamental implications of Eq. 1.6 that motivate the focus in Chapter 2 on noise correla-

\footnotetext{
${ }^{6}$ Noise correlation equal to identity matrix is the theoretically ideal case where there is absolutely zero crosstalk between different channels of an antenna array. Of course, while we strive to approach that ideal, in practice that assumption is next to impossible with today's technology.

${ }^{7}$ This is required to satisfy the identical per channel $B_{1}^{-}$assumption, however it would mean that the 32 channel receiver does not cover the full surface of the sphere. The conclusion of the argument holds regardless.
} 
tion and the push in Chapter 3 and Chapter 4 for higher density receive arrays ${ }^{8}$. Added to these fundamentals is the field effect: simulations have shown that at higher static magnetic fields, a higher number of channels is required to capture the same percentage of the potential SNR [28]. These results further motivate building higher density receivers at ultra-high field.

\subsection{PARAllel IMAGing AND RECONSTRUCTION}

In its simplest form, to accelerate MRI image acquisition, for example in a 2D (two dimensions: phase encoding and readout) Cartesian $k$-space, often only a fraction of the $k$-space lines along the phase encoding direction are acquired. In other words, the frequency domain, $k$-space, is often subsampled along the phase encoding direction.

In its most basic form, Parallel Imaging (PI) refers to the techniques that utilize the additional information of individual elements of the receive array to assist with image reconstruction (often from a subsampled signal) ${ }^{9}$. The subsampling of the signal in $k$-space comes at the expense of aliasing ${ }^{10,11}$ in the image domain. Parallel image reconstruction refers to the mapping from the multi-coil, undersampled signal measured in the frequency domain to an optimally combined, dealiased image. There are various PI reconstruction techniques [33]. The original trailblazer PI reconstruction methods, SiMultaneous Ac-

\footnotetext{
${ }^{8}$ For a more detailed discussion of how the SNR of an array is expected to increase with number of channels, see for example $[27,28,30]$ and references therein. For an experimental demonstration at 3T, see for example [26].

${ }^{9}$ The term parallel probably refers to digitizing and processing the signal from individual coils in parallel as apposed to combining them at earlier stages of the reconstruction pipeline. The term accelerated refers to the reduction in image acquisition time which can be achieved in various ways, including the $k$-space subsampling.

${ }^{10}$ In this context, aliasing broadly refers to a distortion involving the presence of multiple copies of the same object in the image.

${ }^{11}$ For the sake of simplicity, only the PI principles in the case of representation of a simple Cartesian kspace acquisition scheme are described here; in this case, acceleration means skipping "phase encoding lines" and typically results in aliasing. However, if one applies PI on a spiral trajectory, or on a radial trajectory, then there is no more one "phase encoding direction" resulting in aliasing; skipping would rather result in lots of blurring more so than traditional aliasing.
} 
quisition of Spatial Harmonics (SMASH) [34] and SENSitivity Encoding (SENSE) [35], required a prior estimation of individual receive element sensitivity profiles ${ }^{12}$. Very briefly, SMASH in essence relied on a linear weighted combination of receive coil sensitivity profiles to generate shifted $k$-space lines. SENSE used a generalized inverse of the sensitivity matrix to unwrap and combine the aliased images from each channel. The following discussion is focused on introducing those concepts and methods referred to in the subsequent chapters.

\subsubsection{GRAPPA and $g$-Factor}

In their landmark work, Griswold et al. [36] proposed GRAPPA as a method that, in essence, tries to decouple the dealiasing of per-channel images from the SNR-optimized combination of the per-channel ${ }^{13}$ images.

GRAPPA addresses the dealiasing part by filling-in the missing information in the $k$ space on a per channel basis prior to Fourier transforming to the image domain and combining the per-channel images ${ }^{14}$. First, data is acquired with equidistant subsampling of the $k$-space along the phase encoding direction. A central region of the $k$-space, however, is fully sampled (all lines are acquired) and referred to as the Auto Calibration Signal (ACS).

In principle, the process of filling-in the missing $k$-space points is a convolution and involves two steps: (1) determining the set of weights (or convolution kernels) by fitting them to the ACS lines, (2) using these kernels to fill-in the $k$-space via convolution of the kernels with the subsampled signal from all coils. For each target coil, GRAPPA uses data

\footnotetext{
${ }^{12}$ Later versions of SMASH did not require previously collected sensitivity maps. Since SMASH and SENSE are not used in the rest of the thesis, they are discussed only briefly here.

${ }^{13}$ As a side note on terminology clarification, in the context of RF receive arrays, the terms element, channel, coil are used interchangeably to refer to individual resonant loops that together form the array. Coil is sometimes used in literature to refer to the collective array. In the upcoming discussion on the deep learning methods, channel can be used to refer to the depth-wise axis of an n-dimensional matrix (usually the last axis of a numpy ndarray) in the sense that an RGB image has three channels. In that case, it does not necessarily represent a physical coil element.

${ }^{14}$ While there are various implementations with different details, the following description captures the essence of GRAPPA in its principles.
} 

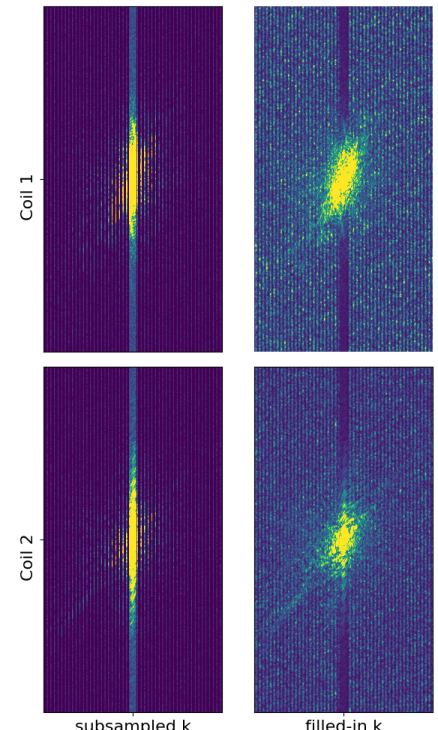

filled-in $\mathrm{k}$
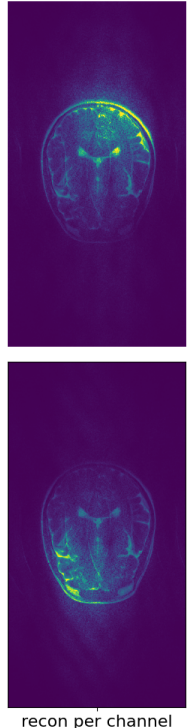

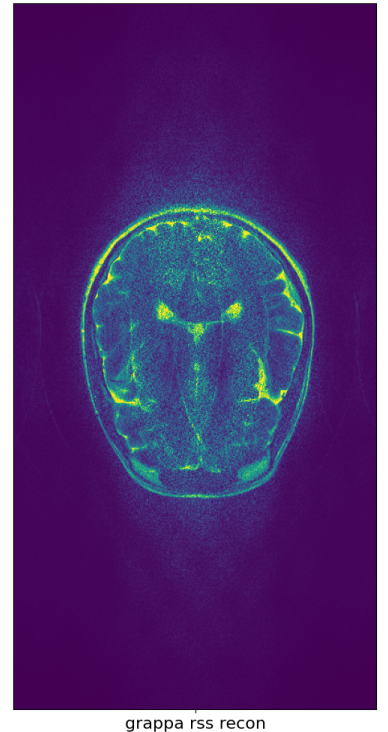

grappa rss recon

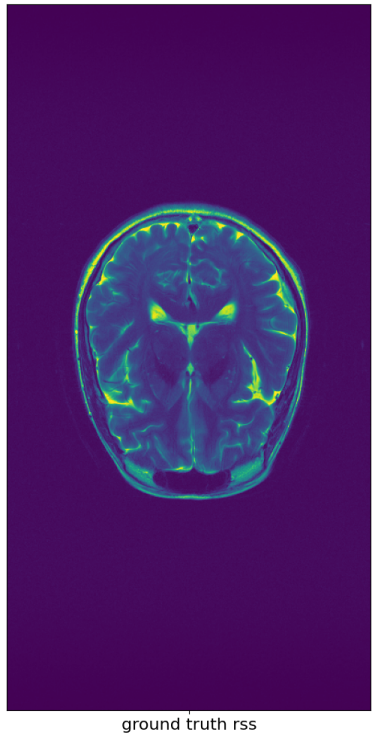

ground truth rss

Figure 1.1: The concept of GRAPPA. It starts from the subsampled, per-channel $k$-space data (left column), estimates the missing points in the $k$-space for individual coils (second column from left), Fourier transforms individual coils to image domain (third column from left), and finally combines the individual images into a single image (fourth column from left). The right column is the fullysampled ground truth reconstruction. Here, a root-sum-of-squares (RSOS) combination is used.

from all individual coils to fit the kernels to the ACS lines in the target coil and then uses these kernels to estimate the missing data of the target coil.

Figure 1.1 illustrates the principles of GRAPPA ${ }^{15}$. It shows a single axial slice of a human brain imaged using a 16-channel receiver, with the acquisition accelerated with a subsampling rate of $R=8$ (i.e. 1/8th of lines acquired) along the phase encode direction $^{16}$. The GRAPPA RSOS reconstruction clearly shows the noise amplification typical of GRAPPA reconstructions of an undersampled acquisition.

The SNR of the image reconstructed from a $k$-space subsampled at the rate of $R$ (i.e. $\mathrm{SNR}_{R}$ ) is related to the $\mathrm{SNR}$ of the image reconstructed from a fully-sampled $k$-space (i.e.

\footnotetext{
${ }^{15}$ The figure is generated using data from the fastMRI [37, 38] dataset.

${ }^{16}$ The reconstruction here used 9x9 kernels. For simplicity, only the magnitude of 2 channels are displayed. The choice of $\mathrm{R}=8$ with only 16 receive channels intentionally accentuates noise amplification for demonstration purposes.
} 
SNR) by [39]:

$$
\mathrm{SNR}_{R}=\frac{\mathrm{SNR}}{g \sqrt{R}}
$$

where $g$ ( $g$-factor or geometry factor), initially introduced by Pruessmann [35], quantifies the noise amplification characteristic of GRAPPA reconstruction and the $\sqrt{R}$ reflects the intrinsic loss due to undersampling ${ }^{17}$. The spatially dependent $g$-factor is a function of the $B_{0}$ field strength, the particular receive array, the reconstruction algorithm, the acceleration rate $R$, and the object being imaged ${ }^{18}$, among other factors [29, 39]. Simulations and experiments have shown that, with all other factors being the same, $g$-factor (the SNR penalty of acceleration) reduces at higher $B_{0}$ magnetic fields (see for example, figure 8 of [27] or figure 1 of [40]). Specifically, the higher $B_{0}$ field strength allows for higher acceleration rates $R$ with less SNR penalty (lower $g$-factors). These results have further encouraged the trend towards ultra-high field MRI.

\subsubsection{RF Arrays and PI $g$-factor}

Quantifying the direct effect of the receive array design and construction on $g$-factor and parallel imaging performance is more challenging ${ }^{19}$. However, experiments converge on the existence of a positive correlation between the number of elements in the receive array and better parallel imaging performance (i.e. reduced $g$-factor), albeit to various degrees, with the advantage of higher density arrays being more pronounced at higher acceleration rates $[1,26,32]$. Simulations of an ideal spherical array also indicate that higher channel count receivers outperform lower channel count receivers in terms of $g$-factor at a constant

\footnotetext{
${ }^{17}$ While Eq. 1.7 is similar to the $g$-factor originally defined in SENSE-type reconstructions, it still holds as a high-level description of the GRAPPA $g$-factor.

${ }^{18}$ The object (a phantom, a human brain, etc) affects the $g$-factor via its size, shape, dielectric properties, and positioning, all of which affect coil loading.

${ }^{19}$ This may be explained by two observations: (1) the manually constructed receive arrays often sustain noticeable electromagnetic deviations from their designs, (2) high fidelity simulations of real-world dense receive arrays are prohibitively complex and time-consuming to validate, at least with the full-wave electromagnetic simulation capabilities available at the time of writing this thesis.
} 
$B_{0}$ field strength [28] and therefore, confirm the experimental expectation of improvement. Other than the number of channels, the noise correlation of the receive array plays a role in determining the $g$-factor [39].

On the other hand, at higher $B_{0}$ fields, a higher number of receive elements is required to capture the same percentage of ultimate-intrinsic SNR as in lower $B_{0}$ fields ([41], see for example figure 2 of [32]). In other words, even in the absence of subsampling, denser receivers are required at higher $B_{0}$ fields. Given the push to higher $B_{0}$ fields that underlies better parallel imaging, the former means that for ultra-high field parallel MRI imaging to realize its full potential, it has to utilize high density receive arrays.

\subsection{DEEP LEARNING FOR MRI RECONSTRUCTION}

The effort to recover the SNR lost to acceleration goes beyond better receiver arrays and higher $B_{0}$ field strength and motivates MRI researchers to try to recover as much SNR as possible in reconstruction as well. The focus on improved reconstruction is pursued in several lines of research. While GRAPPA and SENSE [35] have evolved since their early implementations, compressed sensing [42] techniques have also been applied to the MRI reconstruction problem. Recently, conventional techniques are increasingly used to guide deep learning approaches to the parallel imaging reconstruction problem [43]. Generative adversarial networks [44] are a group of such deep learning models that, having shown promising results in various image-to-image translation tasks, are used in Chapter 5 to address the noise amplification characteristic of GRAPPA.

\subsubsection{Generative Adversarial Networks}

In their landmark work, Goodfellow et al. [44] proposed GAN. GANs are composed of two neural networks trained simultaneously: a generator, $G$, and a discriminator, $D$. The gen- 
erator is tasked with learning the mapping from an input image space to an output image space whereas the discriminator model is trained to estimate the probability that a sample output image is synthetic (generated by $G$ ) or authentic (drawn from the dataset). Goodfellow et al. showed that a pair of $(G, D)$ exists where $G$ captures the input-output mapping of the training data that makes $D$ equally uncertain about its synthetic versus authentic image classification task. The discriminator $D$ is trained to maximize the probability of assigning the correct label (synthetic vs. authentic) to output space images. Simultaneously, $G$ is trained to minimize $\log (1-D(G))$, meaning the generator tries to deceive the discriminator into labeling synthetic images as authentic. This results in solving the following min-max problem:

$$
\min _{G} \max _{D} \mathcal{L}(D, G)=\mathbb{E}[\log (D(x))]+\mathbb{E}[\log (1-D(G(z)))]
$$

where $z$ is a prior noise distribution used as the input to generator, $x$ is the output space, and $\mathcal{L}$ is the total model loss function.

Mirza and Osindero [45] built on the generative model to introduce conditional GANs. Whereas in the original GAN structure, the generator input was a random noise matrix which did not guarantee outcome class, the conditional architecture was constructed to generate synthetic outputs conditioned on class. This is achieved by conditioning the generator and discriminator on some extra information, $y$, for instance by feeding $y$ into both the generator and discriminator as an additional input, and results in the following objective function.

$$
\min _{G} \max _{D} \mathcal{L}(D, G)=\mathbb{E}[\log (D(x \mid y))]+\mathbb{E}[\log (1-D(G(z \mid y)))]
$$

In Chapter 5, I have built upon the original conditional GAN to tackle the parallel image reconstruction and its concomitant noise amplification. In this context, the generator is trained to reconstruct the fully-sampled, unaliased images from undersampled, aliased 
images. 
CHAPTER 2

SELF-DECOUPLING OF RECEIVE ARRAYS

Great find and venue to build non overlapped coils at $10.5 \mathrm{~T}$.

Gregor Adriany - Feb 11, 2019.

One of the primary challenges in building high density receive arrays is that interaction between resonant structures can detune them (i.e. shift the resonance frequency or spoil it altogether $)^{1}$. Decoupling refers to strategies to minimize the interaction between two resonant structures. Self-decoupling is one such strategy that was introduced recently [46].

The following is a discussion of self-decoupling at $10.5 \mathrm{~T}$ and the advantages it offers in designing and constructing high density receive arrays.

\footnotetext{
${ }^{1}$ Tuning a resonant structure refers to adjusting the structure such that its resonant frequency is equal to the desired value, e.g. by modifying the inductor or capacitor values in $f=1 /(2 \pi \sqrt{L C})$ to obtain $f=447 \mathrm{MHz}$, which is the resonant frequency of ${ }^{1} \mathrm{H}$ at $10.5 \mathrm{~T}$. Then, detuning is a shift in the resonant frequency (for whatever reason). It should be noted that while detuning of receiver is undesirable during signal reception, it is intentional/desirable during transmission.
} 


\subsection{INTRODUCTION}

Parallel MRI has become increasingly prevalent in clinical and research settings. Receiveonly RF arrays are critical not only to gain SNR, especially in the vicinity of the receiver coils, but also for parallel imaging performance as they provide the possibility to reconstruct images from subsampled $k$-space and therefore, accelerate MRI acquisitions. Such reconstruction methods rely on the additional spatial encoding provided by array elements and often use sensitivity profiles of each receive element and noise correlation between receive elements. In their seminal work, Roemer et al. [25] presented simultaneous acquisitions using an array of surface coils and an algorithm to combine the signal from each of the receive elements. Roemer suggested that the root-sum-of-square algorithm is the optimum reconstruction solution subject to the noise correlation matrix being proportional to identity matrix. Otherwise, a noise-adjusted root-sum-of-squares reconstruction results in signal-to-noise ratio being inversely proportional to the noise correlation matrix. Furthermore, parallel imaging SNR is penalized by noise amplification ( $g$-factor) which is in turn affected by receiver noise correlation as well as other parameters [39, 47]. Interelement coupling complicates the impedance transformation required for noise-matching the preamplifiers, hence preamplifier noise can reflect back into the coil and be added to the diagonal elements of the noise correlation matrix [32]. Although modern post-processing and reconstruction methods try to deal with receiver noise correlation, the diagonal noise amplification cannot be eliminated [48]. Therefore, SNR and acceleration penalties establish the fundamental motivation to design and build receive arrays with inherently low inter-element noise correlation.

Several decoupling strategies (i.e. methods to mitigate receiver inter-element noise

correlation or crosstalk) have been previously proposed. Roemer et al. [25] demonstrated that overlapping adjacent coils and using low-input impedance preamplifiers minimizes the 
electromagnetic coupling. Preamplifier decoupling relies on a quarter-wavelength transformation to create high series impedance at the coil feed point, thereby minimizing the induced current and inter-element coupling. Inductive decoupling relies on building transformers between adjacent non-overlapping loop elements. Overlap, preamplifier and inductive decoupling were used extensively in the design of receive arrays [32, 49-52]. Lakshmanan et al. [53] proposed the loopole antenna, where segmenting capacitors inside transceive loops were distributed unevenly to cause an unbalanced current distribution in the loop with electromagnetic field patterns resembling that of a dipole antenna. Yan et al. [46] built on the idea of unbalanced impedance and proposed a transceiver selfdecoupling scheme for $7 \mathrm{~T} / 298 \mathrm{MHz}$ where a relatively small RF impedance (e.g. $8.5 \mathrm{pF}$ capacitance) is placed opposite a relatively large RF impedance (e.g. $0.4 \mathrm{pF}$ ) which approaches an open circuit at the RF frequency. This results in the current distribution being unbalanced so that the coil resembles a dipole antenna. However, in contrast to Roemer's work [25], Yan's analysis assumes electric coupling to be limited to coupling via free space and excludes resistive coupling via the conductive sample. While inter-element coupling via the sample can be negligible in the case of loop transmitters far (more than $4 \mathrm{~cm}$ away) from the sample, 3D conformal receiver arrays are generally form-fitting and very close to the sample and are designed to be dominated by body noise [30, 32, 54].

In this work, we present a strategy for receiver self-decoupling at $447 \mathrm{MHz}$ based on our observation that higher frequencies allow for a more balanced capacitive segmentation of receive elements while maintaining acceptable decoupling. We compare performance of self-decoupled receivers, in terms of SNR, with overlap-decoupled loops. Receiver self-decoupling provides inter-element isolation comparable to overlap decoupling while it does not require geometric overlap or interelement transformers or decoupling networks and is therefore much more practical to implement in high density receive arrays. Furthermore, receiver self-decoupling presented here provides higher SNR compared to overlap 
decoupling. It is particularly well-suited to building high density conformal receive arrays. The proposed self-decoupling method, demonstrated in the present work on a pair of receivers, is also being used in building a 32-channel receiver array for human head imaging at $10.5 \mathrm{~T} / 447 \mathrm{MHz}[55]$.

\subsection{Methods}

In the context of MR receive arrays, loop-shaped receive coil elements used to sense $\mathrm{MR}$ signal are rectangular or circular structures ${ }^{2}$ constructed using copper wire and segmented by fixed or variable capacitances so as to become resonant at $f=1 /(2 \pi \sqrt{L C})$. Arranging loop elements into array structures provides SNR and parallel imaging advantages. However, these advantages are compromised by noise and inter-element crosstalk, both of which are reflected in the noise correlation or covariance matrix. In order to mitigate the effects of noise correlation, several decoupling strategies involving overlap, low-input impedance preamplifiers, inter-element transformers, inductive/capacitive networks, and unbalanced current distribution have been proposed before [25, 46, 49, 53, 56-59]. Some of these strategies require additional design and construction complexities which often lead to unintended consequences, including noise amplification, contrary to their raison d'être. For instance, imperfect impedance transformation required in preamplifier decoupling would result in preamplifier noise being projected back into the receive element $[32,60]$. The self-decoupling method presented here takes advantage of the higher frequency $(447 \mathrm{MHz})$ and distributed inductance of receive elements and is particularly appropriate for constructing high-density loop-based receive arrays, primarily for human brain imaging at 10.5T.

Rectangular loops similar in size to those used in the array were modeled in several

\footnotetext{
${ }^{2}$ The shape of a receive element is not necessarily rectangular or circular, but the advantages expected from high density receive arrays especially for parallel imaging at ultra-high field MRI usually impose design requirements that make small rectangular or circular elements arranged in multiple rows a favorite choice for $\mathrm{RF}$ coil designers. In less dense arrays, the optimum shape of the loop element might be different.
} 
scenarios. First, two $5 \times 5 \mathrm{~cm}^{2}$ loops and then, two $2.5 \times 5 \mathrm{~cm}^{2}$ loops were positioned (in simulations) on a flat surface in proximity to a cubic phantom (permittivity $\epsilon_{r}=50$, conductivity $\sigma=0.6 \mathrm{~S} / \mathrm{m}$ to approximate human brain tissue dielectric properties [61] across all simulations). These loops were constructed using American Wire Gauge (AWG)-18 copper wire (diameter $1.02 \mathrm{~mm}$ ) and were divided into four segments using three capacitors and the feed point. Two fixed capacitors and a trimmer capacitor $\left(X_{t r}\right)$, with values in the same range as fixed capacitors, were used inside each loop. The feed circuitry, presented in Figure 2.1, consisted of a detune trap as well as tune $\left(X_{t}\right)$ and match $^{3}\left(X_{m}\right)$ adjustable capacitors. Similar principles were used to construct non-overlapped $10 \times 10 \mathrm{~cm}^{2}$ loops using balanced capacitive distribution on a cylindrical surface at a constant distance from a cylindrical sample $\left(\epsilon_{r}=50, \sigma=0.6 \mathrm{~S} / \mathrm{m}\right)$.

Electromagnetic / circuit cosimulations were performed using CST Studio (Dassault Systemes Simulia Corp., Johnston, RI). Coil elements were modeled in SolidWorks (SolidWorks Corp., Waltham, MA) and imported into CST. Simulations were performed over a frequency range of $2 \mathrm{GHz}$ using the finite difference time domain method to solve Maxwell's equations and were partially accelerated using a GPU. The loops were tuned to $447 \mathrm{MHz}$ (proton resonance at $10.5 \mathrm{~T}$ ) and matched to $50 \mathrm{Ohm}$. In circuit co-simulations, variable capacitors were optimized to tune each loop to the $447 \mathrm{MHz}$ frequency.

Several ElectroMagnetic (EM)/circuit optimization problems were set up with the goal of finding $X_{t r}, X_{t}, X_{m}$ to minimize $S_{21, f}$ conditioned on $S_{11, f} \leq-12 d B$ and $S_{22, f} \leq$ $-12 d B$ where $f=447 \mathrm{MHz}$ is the resonant frequency. Values of $X_{t r}, X_{t}, X_{m}$ were limited to practical ranges guided by bench experiments. Other 3D EM model parameters, including the distance between loop elements and the gap between the coils and the phantom, were kept constant and equal to practical receive array values during optimization. Note that the objective function and the cost (loss) function for these optimizations can

\footnotetext{
${ }^{3}$ Matching a resonant circuit refers to adjusting the circuit components so as to sharpen the resonant peak of the $S_{11}$ intensity-frequency curve.
} 


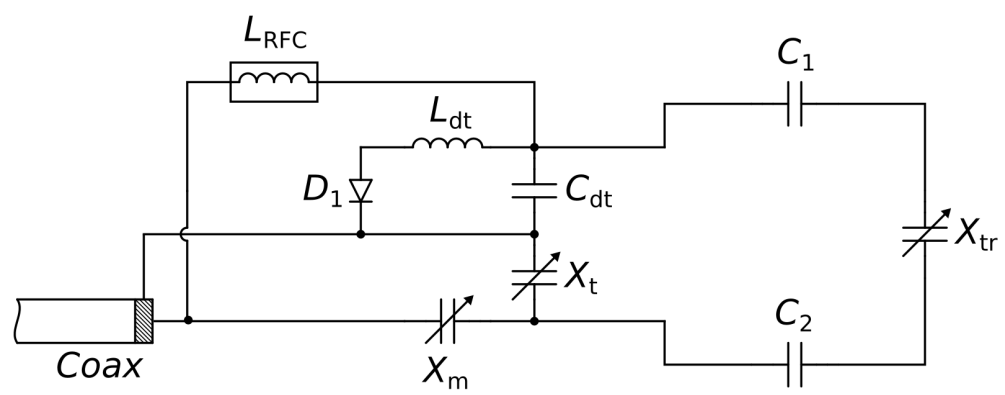

Figure 2.1: Schematic of the feed circuit and loop element, showing the tune $\left(X_{t}\right)$ and match $\left(X_{m}\right)$ capacitors, the detuning trap ( $L_{d t}$ and $C_{d t}$ ), the loop element (fixed $C_{1}$ and $C_{2}$ capacitors and trimmer capacitor $\left.X_{t r}\right)$, and an $\operatorname{RF}$ choke $\left(L_{R F C}\right)$.

\begin{tabular}{|c|c|c|c|c|c|c|}
\hline \multirow{2}{*}{} & \multicolumn{3}{|c|}{$25 \times 50 \mathrm{~mm}^{2}$} & \multicolumn{3}{c|}{$50 \times 50 \mathrm{~mm}^{2}$} \\
\cline { 2 - 7 } & $X_{t r}$ & $X_{t}$ & $X_{m}$ & $X_{t r}$ & $X_{t}$ & $X_{m}$ \\
\hline simulated & 8 & 1.4 & 8 & 6.5 & 2.5 & 3.5 \\
\hline on-the-bench & 8.2 & 1.5 & 8.8 & 7 & 2.5 & 4 \\
\hline
\end{tabular}

Table 2.1: Comparing on-the-bench versus simulated component values.

be defined in various ways. In our experience, incorporating the objective $\left(S_{21, f}\right)$ into the loss function to form a weighted-linear combination of individual L1 norms in the linear (as opposed to $\mathrm{dB}$ ) scale of real and imaginary parts of the S-parameters (as opposed to phase and magnitude) resulted in faster convergence. The trust region method and the Nelder Mead simplex algorithm were used to solve for optimums. It should be noted that in practice (on the bench) such an optimization is straight-forward and is substantially less time consuming compared to the numerical simulations because, on the bench, the results of parameter modifications can be monitored immediately in an analogue manner using a vector network analyzer. Nevertheless, the simulations were useful in validating our bench implementation of self-decoupling (in terms of component values and s-parameters) and comparing self-decoupling with overlap decoupling (in terms of field pattern and SNR). A comparison between the simulated component values versus bench implementation of the same components is presented in Table 2.1.

Numerical results for S-parameters and per-port, complex-valued $\mathrm{H}$-fields at $447 \mathrm{MHz}$ 
were exported to ASCII files. Post-processing and analysis of magnetic field results were performed using customized Python scripts. Complex-valued receive magnetic fields were calculated for each coil element using $B_{1}^{-}=\mu_{0}\left(H_{x}^{*}+j H_{y}^{*}\right) / 2$ where $\mu_{0}$ is the permeability of free space and $x, y$ are Cartesian coordinates orthogonal to the static magnetic field, $B_{0}$. In order to compare the receive field between overlap and self-decoupling methods, two metrics were calculated: Sum of Magnitude (SOM) of the complex combined magnetic fields $S O M=\sum_{\text {sample }} \sum_{\text {channels }}\left|B_{1}^{-}\right|$and the RSOS of magnitudes of each channel's magnetic field $R S O S=\sum_{\text {sample }} \sqrt{\sum_{\text {channels }}\left|B_{1}^{-}\right|^{2}}$, in other words $R S O S=\sum_{\text {sample }} \sqrt{B^{H} B}$ where $B$ is a vector composed of complex magnetic receive fields of each individual channel and $\mathrm{H}$ is Hermitian transpose. Furthermore, RSOS was corrected for noise correlation to calculate noise-correlation-weighted SNR as given by $\mathrm{SNR}=\sum_{\text {sample }} \sqrt{B^{H} \Psi^{-1} B}$ where $\Psi$ is the normalized noise correlation matrix calculated using the simulated complex-valued scatter matrix [27, 32, 62-64]. The summation over sample is intended to collapse spatial maps into a single numeric metric for comparison purposes. These metrics are particularly appropriate here as we are considering receive-only coils, so we are interested in SNR, not transmit efficiency.

\subsection{Results}

Figure 2.2 presents experimental and simulation results for two $10 \times 10 \mathrm{~cm}^{2}$ receive loops at $10.5 \mathrm{~T} / 447 \mathrm{MHz}$. The resulting crosstalk between receive elements is $S_{21}=-12 \mathrm{~dB}$ (Figure 2.2b). Figure 2.3a shows the magnetic field $B_{1}$ of a single excited loop which is consistent with the classic field pattern of a loop at this frequency, implying sufficient isolation. The surface current distribution is almost symmetric with strong current distributed on all segments of the excited loop (and limited current induced on the second loop) (Figure $2.3 \mathrm{~b}$ ), again demonstrating effective interelement isolation. 


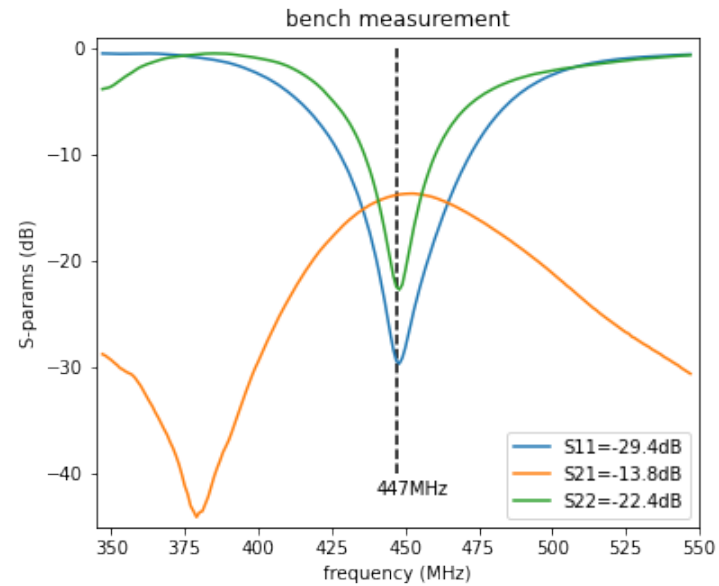

(a)

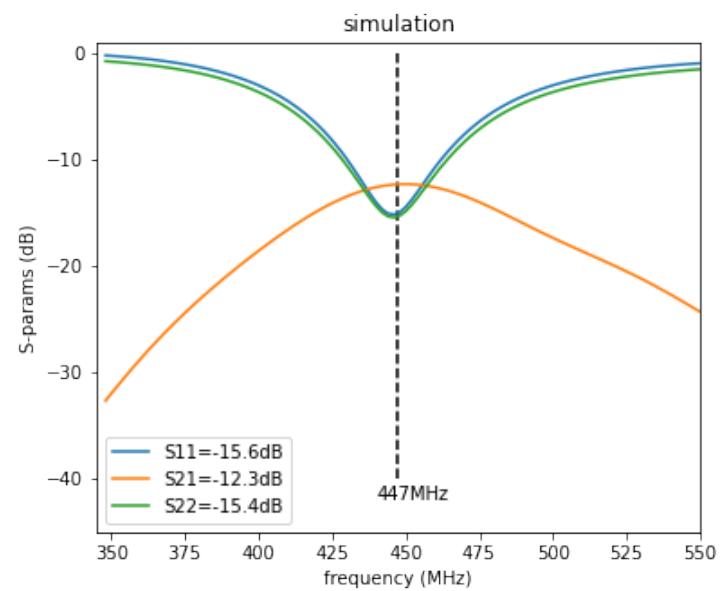

(b)

Figure 2.2: (a) Experimental S-parameters (measured on the bench) demonstrating self-decoupling of adjacent loops, (b) simulated S-parameters. $S_{i j}$ values included are at $447 \mathrm{MHz}$.

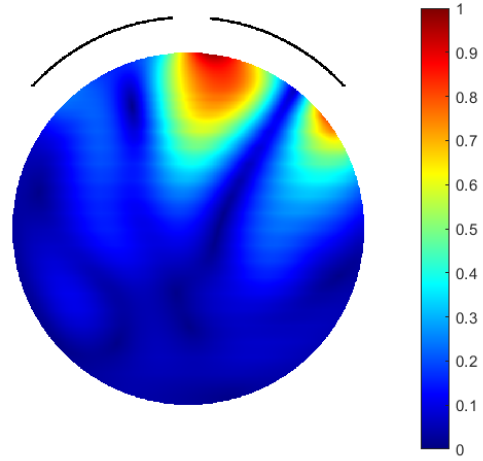

(a)

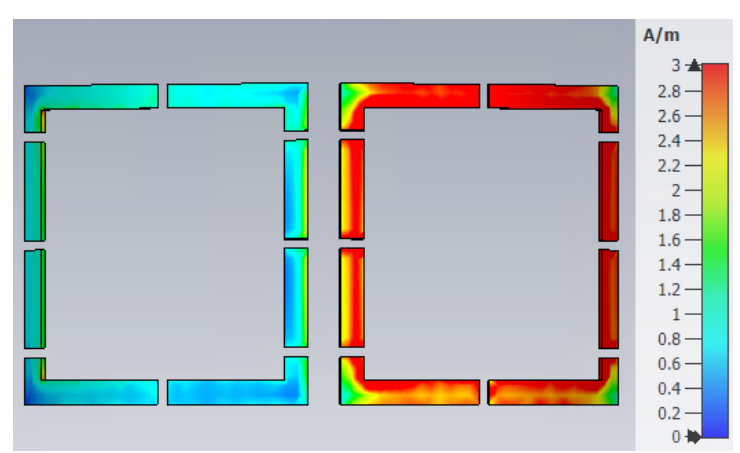

(b)

Figure 2.3: (a) magnitude $B_{1}$ field of one loop (axial, center slice, normalized to 1 , showing the location of the loops as arcs), (b) average magnitude surface current distribution (when only the right loop is excited). 
Simulation results for $25 \times 50 \mathrm{~mm}^{2}$ receive loops demonstrated $-11.2 \mathrm{~dB}$ isolation using the self-decoupling method (compared to $-13.8 \mathrm{~dB}$ using overlap) at $447 \mathrm{MHz}$. In the case of $50 \times 50 \mathrm{~mm}^{2}$ loops, self-decoupling provided $S_{21}=-12.9 \mathrm{~dB}$ interelement isolation (compared to $-16.8 \mathrm{~dB}$ using overlap). These results indicate that the proposed self-decoupling method achieves better than $-11 \mathrm{~dB}$ isolation (in the worst-case scenario) which is sufficient for receive array design given that preamplifier decoupling will further improve the isolation.

\begin{tabular}{|c|c|c|c|}
\hline Loop size $\left(\mathrm{mm}^{2}\right)$ & SOM & RSOS & SNR \\
\hline $25 \times 50$ & 19 & 17 & 10 \\
\hline $50 \times 50$ & 47 & 32 & 26 \\
\hline
\end{tabular}

Table 2.2: Percentage gain in SOM, RSOS, and SNR obtained using self-decoupled loops instead of overlap-decoupled loops (calculated as (self_decoupling_snr - overlap_snr)/overlap_snr * 100) at 10.5T/447 MHz.

Figure 2.4 depicts the $B_{1}^{-}$resulting from two self-decoupled loops and provides a qualitative comparison with $B_{1}^{-}$from two loops of the same size but overlapped. The RSOS combination of receive signals from the two self-decoupled loops shows strong receive signal despite the gap between the two loops (Figure 2.4); a hint of the separation between the two loops is present in the form of two overlapping distributions of $B_{1}^{-}$with two distinct maximal penetration peaks. The two overlapping loops, as expected show more of an overlapping $B_{1}^{-}$distribution. Table 2.2 presents a quantitative comparison between SOM and RSOS of $B_{1}^{-}$and SNR integrated over the sample of self-decoupled and overlapped loops for two sets of loop sizes. Compared to overlapped loops, the proposed self-decoupling method provides $17 \%$ more RSOS $B_{1}^{-}$for the $25 \times 50 \mathrm{~mm}^{2}$ loops and $32 \%$ more RSOS $B_{1}^{-}$for the $50 \times 50 \mathrm{~mm}^{2}$ loops. Furthermore, self-decoupling results in $10 \%$ and $26 \%$ higher noise-correlation-weighted SNR for $25 \times 50 \mathrm{~mm}^{2}$ and $50 \times 50 \mathrm{~mm}^{2}$ loops, respectively. 

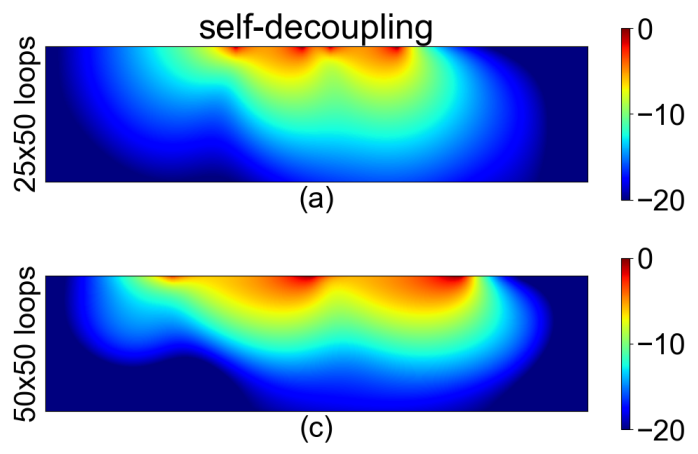
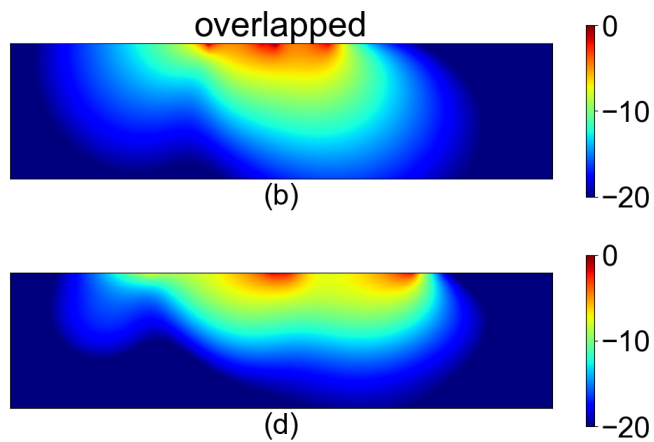

Figure 2.4: Normalized root-sum-of-squares (RSOS) combined $B_{1}$ receive fields of (a) two $25 \times 50$ $\mathrm{mm}^{2}$, self-decoupled loops, (b) two $25 \times 50 \mathrm{~mm}^{2}$, overlap decoupled loops, (c) two $50 \times 50 \mathrm{~mm}^{2}$, self-decoupled loops, (d) two $50 \times 50 \mathrm{~mm}^{2}$, overlap decoupled loops. Numbers are in $\mathrm{dB}$. Phantom size is $50 \times 200 \mathrm{~mm}^{2}$ in all cases.

\subsection{DISCUSSION}

Previous studies have shown that ultimate intrinsic SNR increases with the magnetic field magnitude [40, 41, 65]; the use of high density receive arrays is necessary at UHF to approach ultimate intrinsic SNR [30] available at ultra-high fields and capitalize on the acceleration potential of ultra-high field MRI. However, capturing these potential gains experimentally with increasing number of channels in UHF also depends in the execution of the RF coil array, both in accelerated and un-accelerated imaging. As such, strategies for mitigating interelement coupling and receiver noise play a critical role.

The self-decoupling method presented by Yan et al. [46] introduced a promising new method for decoupling of high density receiver arrays. However, the method at 3T and 7T operating frequencies requires significantly unbalanced distributed capacitors or introduction of inductive circuit elements to achieve the desirable unbalanced current distributions. Primarily because of the higher operating frequency, the same self-decoupling effect was achieved at $10.5 \mathrm{~T}$ with a more uniform capacitive distribution for the loop sizes appropriate for a 32 channel receive array targeting coverage over the human brain. In RF coil validation efforts, achieving a match between the simulated and experimental field maps can be 
easier when current distribution on the loop is more uniform. 


\section{CHAPTER 3}

\section{2-CHANNEL RECEIVE ARRAY AT 447MHZ}

This made my day.

Kamil Ugurbil - Mar 19, 2019.

The self-decoupling method discussed in the previous chapter forms the basis for designing and building high density receive arrays. This chapter deals with the development of a 32 channel receive array for human brain imaging at $10.5 \mathrm{~T}$ using self-decoupling and preamplifier decoupling and compares its performance in terms of SNR with the industry standard 7T-32rx. The materials in this chapter were published in $[3,55]$.

\subsection{INTRODUCTION}

Ultrahigh field magnetic resonance imaging advantages, including higher image resolution, reduced acquisition time, and better signal-to-noise ratio, have opened new opportunities for advancement of various clinical and basic research projects. Applications of primary interest that rely on exploiting these UHF advantages include fMRI studies, brain connectivity mapping using diffusion-weighted MR imaging (dMRI), and anatomical imaging. 
The push for UHF MRI is predicated primarily on the premise of significant ultimate intrinsic SNR [27, 41, 65, 66] gains and less SNR penalty with parallel imaging acceleration $[1,17,40,67,68]$ at higher $B_{0}$ field strengths. However, UHF MRI presents significant new technical challenges [5, 6, 69-71]; a critically important technology that enables acquisition strategies that addresses some of these challenges, such as reducing echo-train lengths in fast acquisition schemes like Echo Planar Imaging (EPI), is parallel imaging [29] using high density receive arrays $[1,30,32,72]$.

Acceleration via parallel imaging comes with a penalty in SNR quantified in terms of noise amplification or g-factor [35]. Increasing the $B_{0}$ field strength has been shown to mitigate the SNR penalty attributed to acceleration [29, 40, 73], hence affording better parallel imaging performance. However, parallel imaging performance depends significantly on receiver array noise correlation $[39,47]$ as well which needs to be addressed with careful decoupling strategies. Increasing receive array density, on the other hand, exacerbates interelement coupling (noise correlation) and dominance of electronics noise with smaller loops $[26,32,54]$. Therefore, developing receiver arrays at $10.5 \mathrm{~T}$, the highest field strength currently available for human imaging, requires incorporation of novel decoupling techniques that are effective at $447 \mathrm{MHz}$ (the proton resonance frequency at 10.5T), in order to fully exploit the superior SNR and acceleration potential of the higher magnetic field.

Here, we implement the novel receiver self-decoupling technique to build a 32-channel receive array without using within-row (axial) overlap [25], explicit inter-element decoupling networks $[50,56,57]$, or highly unbalanced current distributions $[46,53]$. The primary contributions of this work are (1) building a high density, self-decoupled 32-channel receive array for $10.5 \mathrm{~T} / 447 \mathrm{MHz}$ for the first time, and (2) demonstrating SNR and parallel imaging gains at $10.5 \mathrm{~T}$ compared to $7 \mathrm{~T}$. The self-decoupled 32-channel receive array $(10.5 \mathrm{~T}-32 \mathrm{Rx})$ provided substantial experimental peripheral (corresponding to cortical regions in a human head) and central SNR gains compared to an industry-standard 32- 
channel receive array at 7T. Furthermore, parallel imaging performance at $10.5 \mathrm{~T}$ was superior to that of $7 \mathrm{~T}$, with $10.5 \mathrm{~T}-32 \mathrm{Rx}$ providing acceleration performance comparable to a 64-channel array coil at 7T. Our results also confirm the clear advantages of higher $B_{0}$ field in terms of SNR and PI performance.

\subsection{Methods}

\subsubsection{The transmitter}

The primary focus of this chapter is on the contribution of receiver coil self-decoupling and $B_{0}$ field strength to SNR and parallel imaging performance. Therefore, the transmitter design is covered here only briefly, with its detailed characterization published separately [74]. A 16-channel transmitter comprising two rows of 8 inductively decoupled rectangular loops was used $[49,74]$. The 2-row design of the transmitter array $[49,75,76]$ has the potential of increasing degrees of freedom in Parallel Transmit (pTx) RF pulse design especially for Specific Absorption Rate (SAR) control [77-79]. In order to minimize transmit-receive interaction, the transmitter was actively tuned, i.e. a PIN diode circuitry was used to tune the transmitter during signal transmission, leaving it off-resonance during reception. The 10.5T MR scanner is currently operated under an Food and Drug Administration (FDA) Investigational Device Exemption (IDE) which requires that all RF coils planned for human use are approved by the FDA. The procedure to obtain necessary approvals for in-vivo human brain imaging using this transmitter together with a receive coil inserted in it is currently ongoing.

The receiver design and construction involved several steps: (1) two loop simulations with the primary purpose of verifying and characterizing the self-decoupling concept at 10.5T (discussed in Chapter 2), (2) prototyping an 8-channel subset of the 32-channel receiver with the primary purpose of assessing noise correlation, (3) building the final 32- 
channel receiver. The later two steps are discussed in the following sections.

\subsubsection{The 8-channel prototype}

A prototype composed of eight channels, arranged in four rows to be representative of the final layout, was initially built and tested on the bench prior to measurements both on the bench and in the $10.5 \mathrm{~T}$ scanner with the primary purpose of assessing the noise correlation. A 16 AWG silver-coated wire was used to construct the loops. Lumped capacitor values employed in the loops were $3.3 \mathrm{pF}, 4.7 \mathrm{pF}$, and $6.8 \mathrm{pF}$. One trimmer capacitor with a value range of 8-20pF or 2-6pF (SGC3S300NM or SGC3S060NM, Sprague-Goodman, NY, USA), included inside each loop, was carefully adjusted to decouple the loops in each row based on their scatter matrix parameters. The values of the larger trimmers were measured to be in the range of $8.5-15 \mathrm{pF}$ after adjustment. The feed board, including the active detuning circuitry, and the preamplifier board were similar to those presented in a previous ISMRM abstract [52].

Primarily, these prototype experiments were aimed at addressing the noise correlation issue. In the initial experiments with the 8 channel receive array prototype, noise correlation of the prototype was measured to reach a maximum of 0.67 . Several experiments aimed at improving this noise correlation were performed using that prototype both on the bench (measuring $\mathrm{S}$ parameters) and in the MR scanner (measuring the noise correlation matrix) while modifying the preamplifier positioning and cabling arrangements.

First, the effect of the spacing of preamplifier boards on the noise correlation was investigated. Two configurations, one with the preamplifiers close to each other and another with preamplifiers far from each other, were constructed. The noise correlation matrix was measured in the MR scanner in both configurations. No meaningful difference in noise correlation was observed in these two configurations, ruling out the possibility of interaction between preamplifier boards (and the baluns at the output of the preamplifiers). 
Second, the effect of cable traps on the noise correlation was investigated. On the bench, having multiple cable traps on a single coaxial cable would result in the S-parameter of individual cable traps showing split resonances, which indicated the interaction between these cable traps. This observation was taken into account while building the 32 channel receiver by intentionally tuning some of the cable traps slightly off of the resonance frequency of $447 \mathrm{MHz}$, so the traps still provided some suppression at $447 \mathrm{MHz}$ but given the off-resonance tuning, had less of an interaction with each other and with the adjacent loop elements.

Third, experiments with the routing of coaxial cables feeding the preamplifiers were aimed at measuring and minimizing the interaction between transmit and receive channels. Using a single transmit element, the $S_{i j}$ from the transmitter to each of the eight channels of the prototype was measured on the bench while the coaxial cables feeding the preamplifiers were moved to position them along the diagonal, edge or center of the single transmit element. It was observed that routing these coaxials along the center of the transmit element results in less transmit/receive interaction compared to other configurations.

Overall, these experiments converged on stacking the preamplifiers (on both sides of planar substrates, as seen in the as-built coil photo), using a single trap at the center of each coaxial cable, and running these coaxial cables along the center of transmitter loops. These prototype experiments reduced the noise correlation measured in the MR scanner to maximum of $\sim 0.35$.

\subsubsection{The 32-channel receiver}

A close-fitting receive former (helmet) was designed while considering physical constraints imposed by the transmitter (inner diameter of transmitter is $28.5 \mathrm{~cm}$ ), the dimensions of which were directed by a $41 \mathrm{~cm}$ ID head gradient coil that will be used in the future. The shape of the former was optimized based on previous helmet designs, numerical model of a 


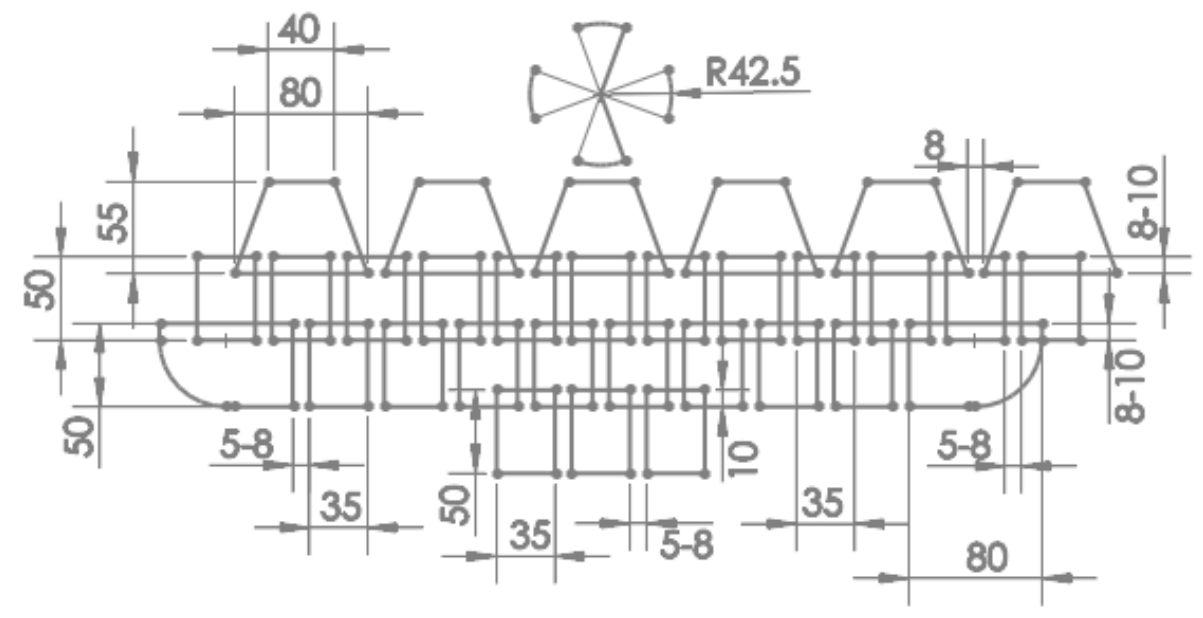

Figure 3.1: Flattened layout of the 32 channel receiver (approximate dimensions in $\mathrm{mm}$ ).

human head, and consideration of average range of head sizes. A structure for mechanical support of the preamplifiers and cables was designed to be mounted as an additional part on top of the head former. A visual channel at the level of the eyes was designed to accommodate the needs of future fMRI studies, which often involve visual stimulus presentation or eye tracking. The distribution of preamplifier substrates (i.e. 3D printed mounting supports for the preamplifiers) was driven by cable routing decisions, discussed previously, as well as preamplifier interactions and directional sensitivity relative to the $B_{0}$ direction.

The 32Rx receive array is composed of 31 loops, divided into four rows along the z-axis, and one cloverleaf element covering the top. The layout of the loops, presented in Figure 3.1, allowed for partial overlap among elements of the different rows (8-10 mm) and gaps between neighboring loops within each row $(5-8 \mathrm{~mm})$; the self-decoupled loops with gaps in the axial (x-y) plane are included here to provide an SNR advantage compared to overlapped loops. This results in a high-density receive array designed to rely primarily on self-decoupling. Each row of loop elements was shifted (by a half loop) compared to the neighboring rows (i.e. rotated azimuthally in the real coil). This self-decoupled design substantially reduced the construction complexity that would arise from overlapping 
adjacent loops within each row or using decoupling networks or transformers between loop elements and contributed to the SNR improvements. Overlap along the z-axis (along the center of the MR scanner bore) was maintained to further improve SNR via increased channel density.

A key consideration in designing the layout was a primary focus on the visual cortex, driven by a large array of vision neuroscience projects conducted at CMRR that can greatly benefit from higher field strength; this, in turn, motivated an increase in density in the posterior array for the rows that will be facing the occipital lobe, at the expense of a reduced density at the top (six loops) and bottom (three loops) rows. Loop elements with the plane of the loop aligned perpendicularly to the z-axis would have compromised sensitivity; therefore, a cloverleaf element, rather than loop elements, was placed at the top of the coil, resulting in a Poynting vector perpendicular to the z-axis [80]. The cloverleaf element was composed of two figure-8 (bow-tie shaped) loops placed perpendicular to each other; the outputs of the figure- 8 elements were combined in hardware as a quadrature pair to form a single receive channel. The as-built receive array is presented in Figure 3.2.

\subsubsection{Noise correlation}

On the bench, the scatter matrix $\left(S_{i j}\right)$ was measured using a calibrated 16-port vector network analyzer (VNA, ZNBT8, Rohde \& Schwarz). Bench measurements between all coil pairs using the 8-channel prototype showed little interaction between elements far from each other. Therefore, similar $S_{i j}$ bench measurements for the completed 32-channel array were limited to adjacent coils in the same row or overlapping coils from different rows, considered to represent worst-case scenarios based on the prototype measurements. Later, the full noise correlation matrix for the 32-channel receiver was measured in the scanner. Measurements were done after tuning, matching, and self-decoupling the coils and both prior to and after adding preamplifiers. Self-decoupling of adjacent loops within each row 


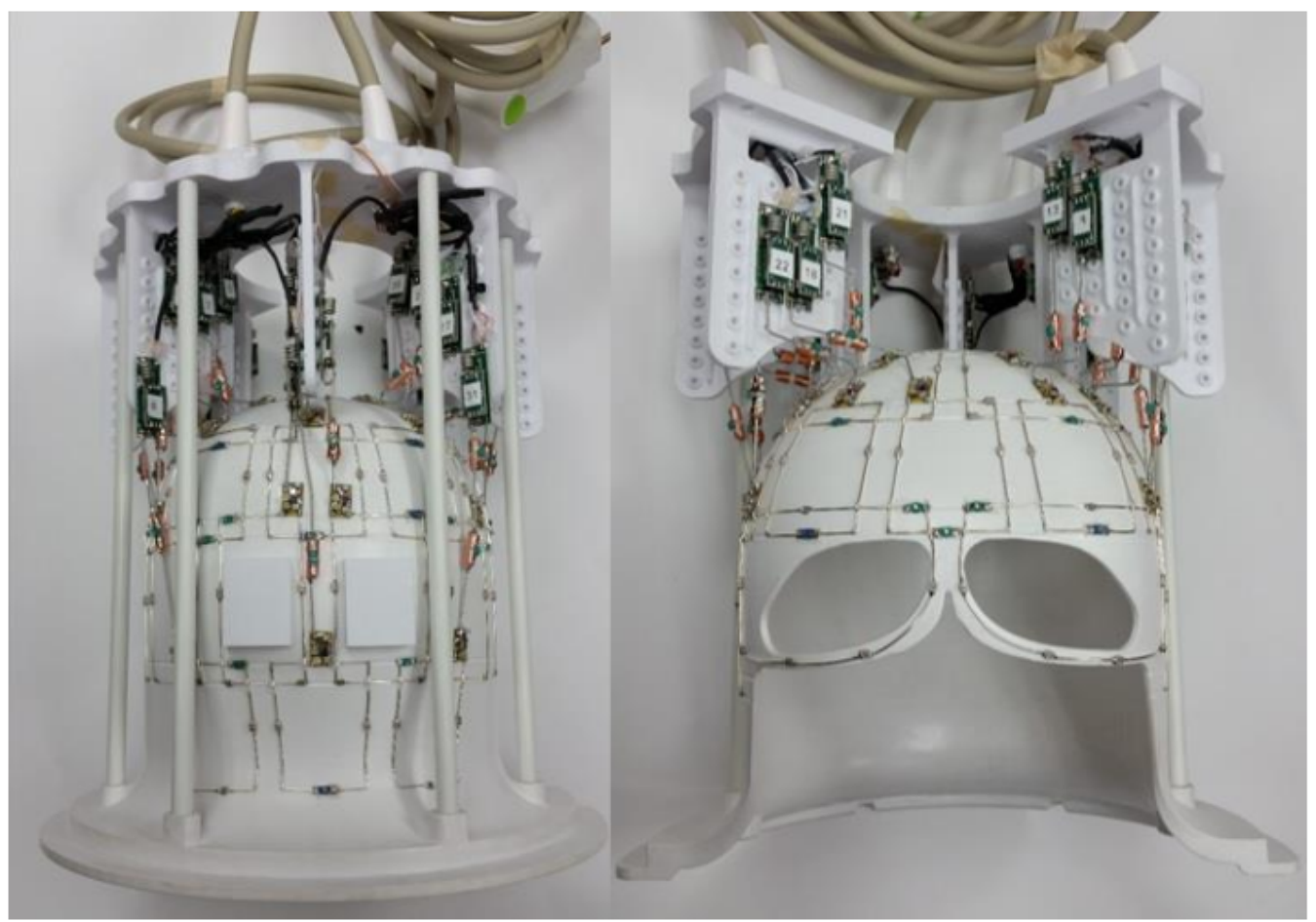

Figure 3.2: Photograph of the 32-channel receiver as built.

provided robust inter-element isolation. Low noise preamplifiers (WMA447A, WanTcom Inc., Minneapolis, MN) with input impedance of $1.5 \Omega$ and noise figure of $0.45 \mathrm{~dB}$ were used for preamplifier decoupling. Preamplifiers were mounted on a ground plane which helped minimize interactions with transmitter/receiver elements and preamp oscillations. To reflect the actual use case, $S_{i j}$ measurements were done with all receive elements in the tuned state.

Using the 8-channel prototype, preamplifier interactions and coaxial cable interactions were investigated to characterize the effects of stacking preamps/coaxial cables close to each other as well as cable routing. Noise correlation matrices for various cabling, preamplifier configurations, and cable trap locations were measured inside the 10.5T MR scanner with the 8-channel prototype. While we did not observe any improvement in noise correlation by distancing the preamplifier boards from each other, having a single cable trap at the center of each input coaxial cable improved noise correlation. Furthermore, routing 
the preamplifier input coaxials along the center of transmit loops reduced transmit/receive interaction.

Coaxial cables were isolated using self-shielded input cable traps to suppress shieldcurrent-induced noise [81, 82]. Traps were carefully tuned after being installed on preamplifier input coaxial cables; however, several cable traps were intentionally tuned slightly off-resonance to mitigate their interaction with resonant loops of the receiver or transmitter [49]. These input cable traps are resonant structures constructed using a trimmer capacitor (8-30pF, SGC3S300NM, Sprague-Goodman, NY, USA) soldered to copper tape wrapped around a dielectric cylinder mounted on the outer conductor of preamplifier input coaxials (with the copper tape soldered to the outer conductor). One further s-matrix measurement was made with output cable traps detuned to investigate potential crosstalk between those resonant structures as well.

\subsubsection{Transmit-receive interaction}

Design of routing paths for preamplifier input coaxial cables was driven by noise correlation and receive-transmit interaction considerations. Coaxial cables may present a significant conductor barrier to the transmitter. Based on previous experience with loop transmitter designs [50], knowledge of transmit field patterns [74], and prototype experiments explained above, we observed that collecting coaxial cables in five paths along the $\mathrm{z}$-axis parallel to the center of the transmit loops would result in minimum $\mathrm{Tx} / \mathrm{Rx}$ interaction.

Measurements of the receive-transmit interaction were performed both on the bench and in the scanner. On the bench, scatter matrix parameters of the transmitter were monitored before, during, and after insertion of the receive array, while the receiver was actively detuned using DC supply to PIN diodes [52] and loaded with a human head shaped phantom. In the scanner, transmit field maps were acquired in two configurations: first, with the 16-channel transmitter and 32-channel receiver as an ensemble of transmit-only and 
receive-only arrays, second, with the 16-channel transmitter used as a transceiver in the absence of the 32-channel receiver. Relative transmit $B_{1}^{+}$maps were acquired using a small flip angle multi-slice Gradient Echo (GRE) sequence with magnitude images from sequential single channel transmissions normalized by their sum [83]. Absolute transmit $B_{1}^{+}$maps were then generated by normalizing relative transmit maps by $\sin (\alpha(\vec{r}))$ where $\alpha(\vec{r})$ is the actual flip angle as a function of spatial coordinate $(\vec{r})$ obtained via 3D GRE Actual Flip Angle Imaging (AFI) acquisitions obtained with all channels transmitting [83-85]. Absolute transmit field maps were acquired both with and without the receive array in place, with the transmitter used as a 16-channel transceiver when the 32-channel receive array was not inserted.

\subsubsection{SNR and $g$-factor}

\section{Data acquisition}

Data were acquired on a 10.5T MRI (Siemens Healthcare, Erlangen, Germany) system using a phantom with relative permittivity $\epsilon_{r} \simeq 47.25$, conductivity $\sigma \simeq 0.65 \mathrm{~S} / \mathrm{m}$ at 447 MHz as measured with a DAKS-12 (SPEAG, Zürich, Switzerland) to approximate human brain tissue properties [61]. All SNR measurements were replicated two times, once with the $16 \mathrm{Tx} / 32 \mathrm{Rx}$ coil described above at 10.5T, then with an industry-standard $1 \mathrm{Tx} / 32 \mathrm{Rx}$ coil (Nova Medical Inc., Wilmington, MA) at 7T using protocols, acquisition parameters, setups, and data analysis pipelines similar to those at 10.5T.

Relative transmit field maps were obtained using a series of small flip angle multislice gradient echo (2D GRE) sequences, pulsing on one transmit channel at a time [83, 86]. Typical sequence parameters were seven $5 \mathrm{~mm}$ thick axial slices (with center slice being isocenter), $\mathrm{TR}=100 \mathrm{~ms}, \mathrm{TE}=3.5 \mathrm{~ms}$, Flip Angle $(\mathrm{FA})=10^{\circ}$, pixel bandwidth $=300$ Hz/pixel. AFI [85] maps were acquired (3D, TR=75ms, TE $=2.0 \mathrm{~ms}$, nominal $\mathrm{FA}=50^{\circ}$ ) with all channel transmitting together. SNR data were acquired at approximately fully 
relaxed longitudinal magnetization, with a multi-slice GRE sequence, with TR=7000ms$10000 \mathrm{~ms}, \mathrm{TE}=3.5 \mathrm{~ms}, \mathrm{FA}=80^{\circ}$, pixel bandwidth $=300 \mathrm{~Hz} /$ pixel. This was followed by a noise scan which was identical to the $\mathrm{SNR}$ sequence except for $\mathrm{FA}=0^{\circ}$ (no RF pulse), $\mathrm{TR}=70-100 \mathrm{~ms}$ [87]. When measuring AFI maps for SNR normalization at 10.5T, a 16Tx, circularly polarized (CP-like) transmit field $B_{1}^{+}$phase shim setting was calculated for the 16 transmit channels allowing for acceptable $B_{1}^{+}$efficiency over a large fraction of the phantom $[71,88]$.

\section{SNR and g-factor calculations}

The noise correlation matrix was calculated based on complex noise data (obtained in the absence of RF pulsing) and used to decorrelate the SNR data before they were combined using root sum-of-square method $[25,63]$. In the steady state, signal intensity in a GRE sequence is proportional to $\rho\left(1-E_{1}\right)(\sin \theta) /\left(1-E_{1} \cos \theta\right) E_{2}$ where $\rho$ represents proton density, $E_{1}=\exp \left(-T_{R} / T_{1}\right), E_{2}=\exp \left(-T_{E} / T_{2}^{*}\right)$ and $\theta$ is the spatially varying, voltagenormalized actual flip angle. With $T_{R} \gg T_{1}$ it follows that $E_{1} \ll 1$ which results in $\mathrm{SNR} \propto \rho \sin (\theta) E_{2}$. SNR maps were normalized by $\sin (\theta)$ derived from AFI maps, voxel size, number of acquisitions, number of samples along the read-out and phase-encoding directions, and bandwidth to make SNR calculations comparable across experiments [84]. SNR ratios were further normalized by $T_{2}^{*}$ values which were measured at $34 \mathrm{~ms}$ and $28 \mathrm{~ms}$ at $7 \mathrm{~T}$ and $10.5 \mathrm{~T}$, respectively. The noise figure from receiver chain of the MR scanners (excluding RF coil and preamplifiers) was not reflected in these calculations. Noise amplification in accelerated images was quantified in terms of $g$-factor [35] and calculated as

$g=\mathrm{SNR} /\left(\mathrm{SNR}_{R} \times \sqrt{R}\right)$ where $\mathrm{R}$ is the acceleration factor and $\mathrm{SNR}_{R}$ is the accelerated SNR calculated based on fully sampled acquisitions that were retrospectively undersampled. In order to be able to compare $g$-factor numbers of the $10.5-32 \mathrm{Rx}$ presented here with previously published [1] g-factors of 7T-32Rx and 7T-64Rx, the same acquisi- 
tion and post-processing pipeline used for 7T-32Rx and 7T-64Rx were maintained in our experiments with the 10.5T-32Rx.

\subsection{RESULTS}

\subsubsection{Receiver noise correlation and $\mathrm{Tx} / \mathrm{Rx}$ interaction}

The inter-element isolation was measured prior to preamplifier decoupling as $S_{21}$ at the resonance frequency of $447 \mathrm{MHz}$; these values were on average in the range of 11-12dB for self-decoupled adjacent coils in the same row, 12-15dB for partially-overlapped coils from different rows and in the range of $20-30 \mathrm{~dB}$ for distant neighbors, demonstrating effective self-decoupling of the 32-channel receive array without the need for overlap in each row, explicit transformer decoupling, or unbalanced capacitive distribution. Preamplifier decoupling further reduced crosstalk between receive array elements to negative $35-40 \mathrm{~dB}$.

The noise correlation matrix measured inside the 10.5T MR scanner resulted in maximum noise correlation of 0.37 (Figure 3.3). We attribute this to the novel self-decoupling technique, experimentally optimized cable routing (relative to transmit elements to minimize shield-current-induced noise and transmit field distortion) and cable trap locations (to minimize trap interference with receive element resonances).

Figure 3.4 shows power-normalized transmit field maps (in $\mu T / \sqrt{W}$ ) measured in the 10.5T scanner using the 16-channel transmitter with and without insertion of the $32 \mathrm{Rx}$ receive array, with the phases of the different channels on the transmitter set so as to generate a circularly polarized $B_{1}^{+}$field, which results in the center bright $B_{1}^{+}$maps [71]. These maps demonstrated less than $10 \%$ distortion of the transmit field upon inserting the receive array; as such, characterizing the limited transmit field change following the insertion of the receive array. This characterization allows for streamlining safety validation studies by obviating the need for inclusion of the receive array in electromagnetic simulations of 


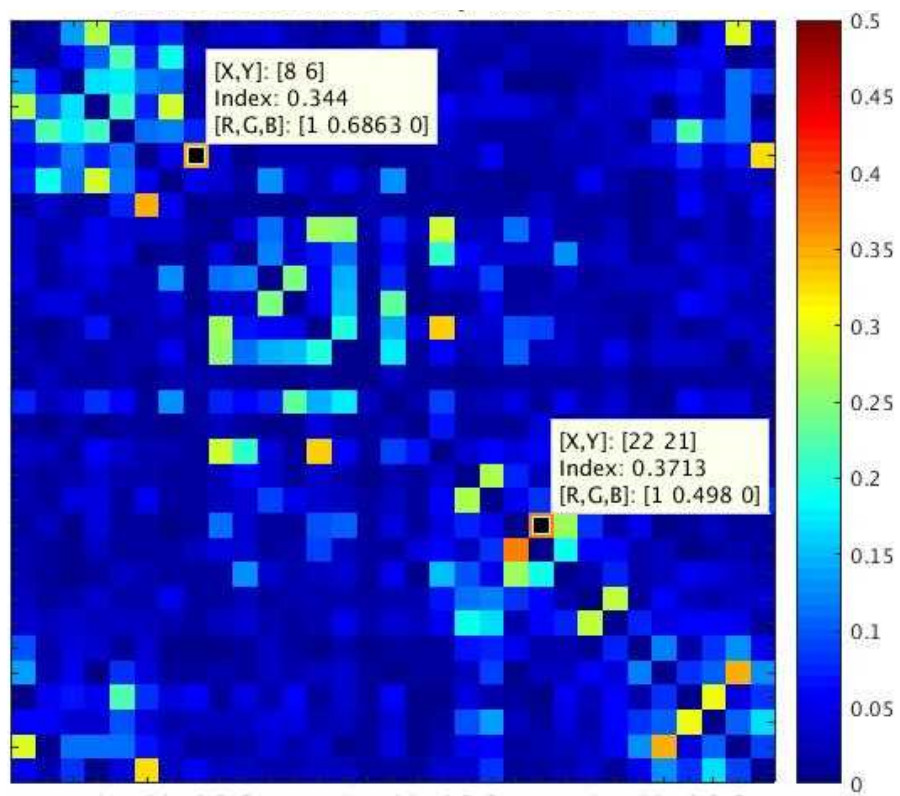

Figure 3.3: Experimental noise correlation map for the 10.5T-32Rx receiver demonstrating effective decoupling of receive elements (maximum noise correlation is 0.37 ).

SAR.

\subsubsection{SNR and $g$-factor}

Figure 3.5 illustrates experimental unaccelerated SNR comparisons in axial slices obtained with identical protocol, experimental setups, and analysis pipeline using a 32-channel receiver at 7T (7T-32Rx) and a 32-channel receiver at 10.5T (10.5T-32Rx). The phantom and Region of Interest (ROI)s used for SNR comparison are depicted in Figure 3.6. SNR comparisons were made by averaging over a central region and a peripheral region defined by a circular boundary at depth "d" into the phantom (Figure 3.6b). Table 3.1 presents the SNR ratios for various peripheral versus central segmentations parametrized by the depth "d" of the central/peripheral boundary. SNR comparison demonstrated 1.46 times central and 2.08 times peripheral SNR gains at $10.5 \mathrm{~T}$ with $\mathrm{d}=20 \mathrm{~mm}$. Figure $3.6 \mathrm{c}$ provides unaccelerated 3D (axial, coronal, sagittal) SNR comparisons between 10.5T-32Rx and 7T-32Rx in the central slices. 

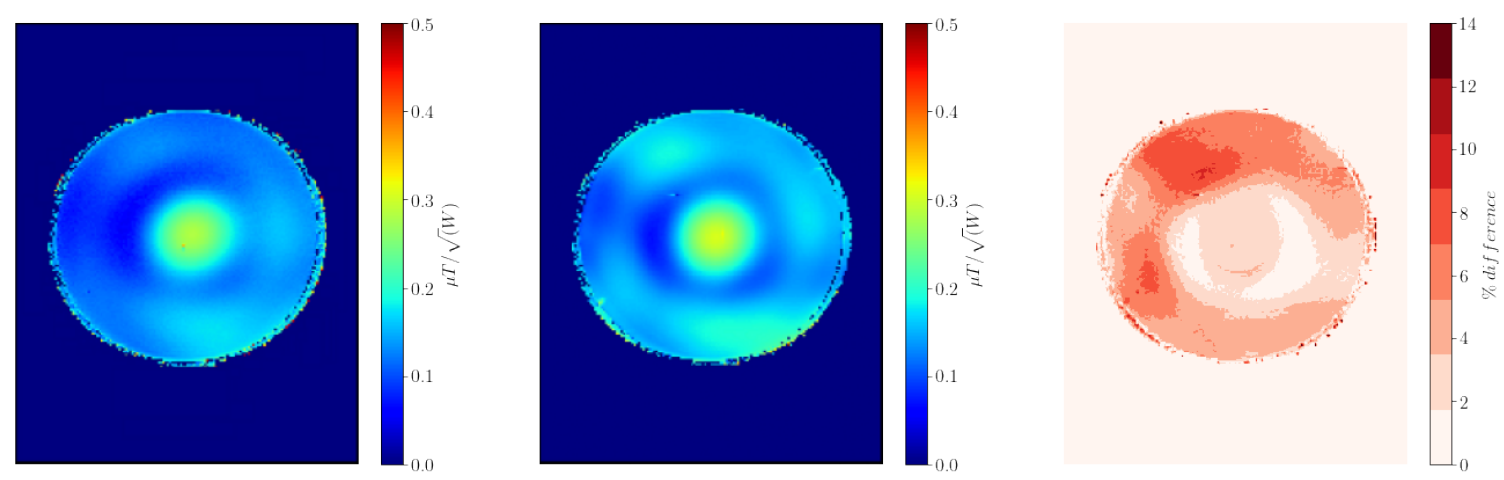

Figure 3.4: Comparing experimental normalized transmit field maps of (left) 10.5T-16Tx used as transceiver without the presence of 32Rx versus (center) $10.5 \mathrm{~T}-16 \mathrm{Tx} / 32 \mathrm{Rx}$, with (right) the percent difference demonstrating the effect of Rx insertion to be limited to $10 \%$.

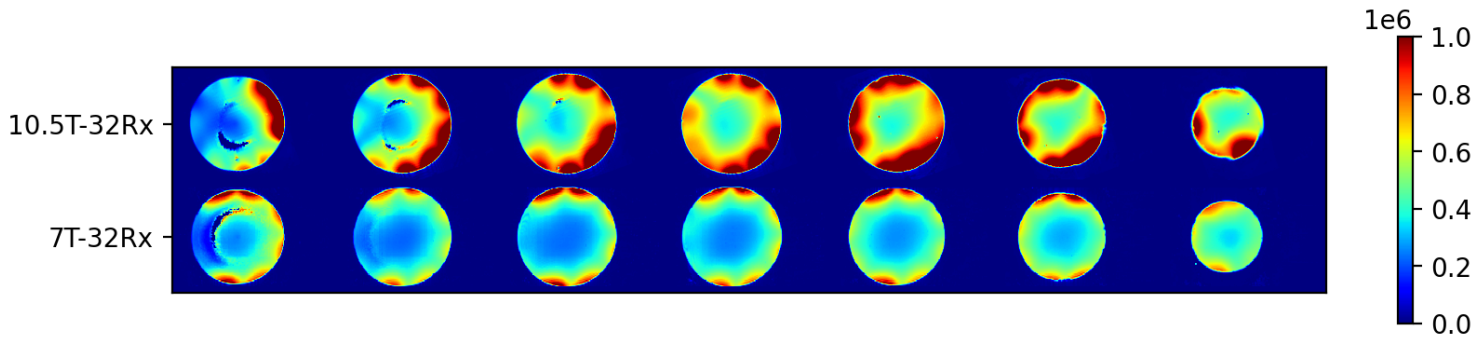

Figure 3.5: Comparing normalized SNR of the 10.5T-32rx (top row) and industry standard 7T-32rx (bottom row) on eight axial slices. Local defects (the crescent-shaped signal loss in first three slices from right) are due to specific transmit B1 shim. The dark center (in the first two slices from right) is an oil reference. Numbers in $\sqrt{\mathrm{Hz}} / \mathrm{ml}$. 


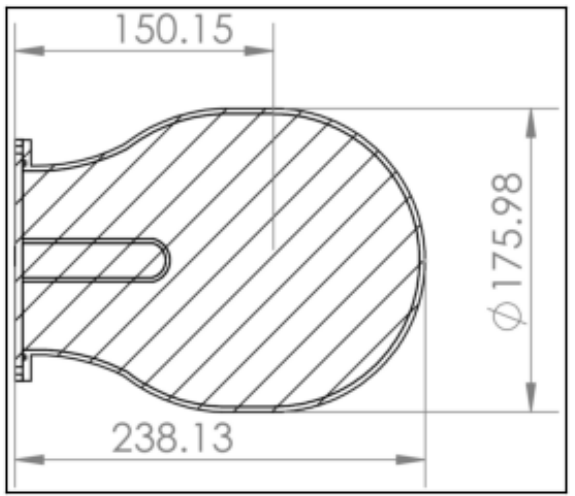

(a)

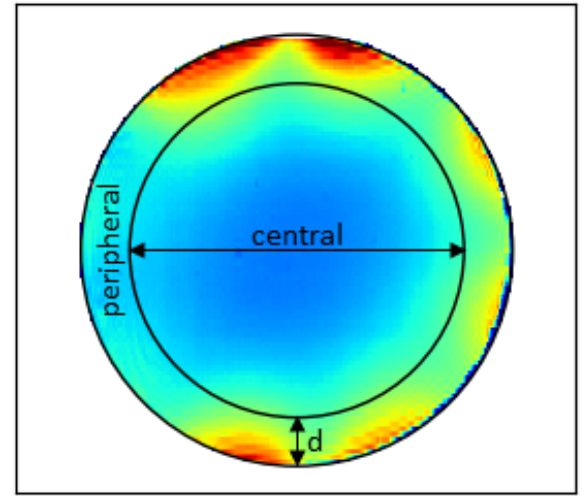

(b)

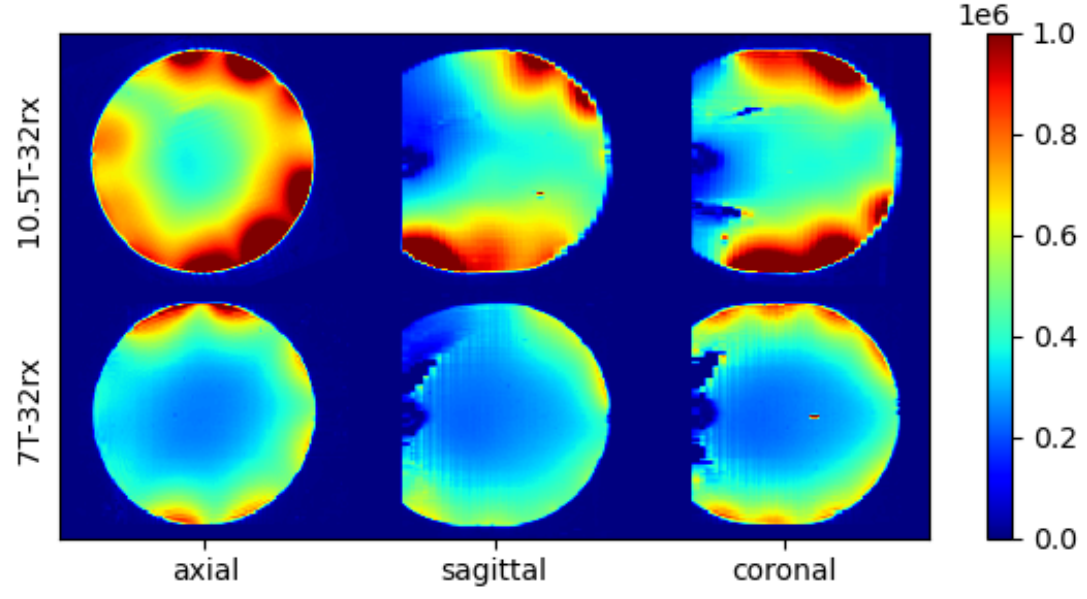

(c)

Figure 3.6: (a) Phantom cross section; the cylindrical tube is an oil reference. (b) axial cross section showing the central versus peripheral regions demarcated by d, depth into the sample. (c) comparing SNR from the 10.5T-32rx (top row) with SNR from the 7T-32rx (bottom row) in three orthogonal planes (SNR in $\sqrt{\mathrm{Hz}} / \mathrm{ml})$. 


\begin{tabular}{|c|c|c|c|}
\hline Depth (d, mm) & Peripheral SNR ratio & Central SNR ratio & $\begin{array}{c}\text { Overall Average } \\
\text { SNR ratio }\end{array}$ \\
\hline 30 & 1.95 & 1.44 & 1.81 \\
\hline 25 & 2.00 & 1.46 & 1.81 \\
\hline 20 & 2.08 & 1.46 & 1.81 \\
\hline 15 & 2.25 & 1.47 & 1.81 \\
\hline 10 & 2.58 & 1.47 & 1.81 \\
\hline
\end{tabular}

Table 3.1: Ratio of the average 10.5T-32Rx SNR divided by average 7T-32Rx SNR for various values of $d$ (peripheral vs central boundary, delineated in Figure 3.6b).

The overall average SNR gain using the $10.5 \mathrm{~T}-32 \mathrm{Rx}$ was $81 \%$ compared to the $7 \mathrm{~T}$ 32Rx. Expressed as an exponent $n$ of the static magnetic field strength (i.e. as $B_{0}^{n}$ ), the overall average $n \simeq 1.5$, and in the periphery $n \simeq 1.8$ (using SNR ratios for $\mathrm{d}=20 \mathrm{~mm}$ ). This enhanced SNR is primarily attributed to higher static magnetic field $B_{0}$ strength as well as noise mitigation and SNR advantages provided by the self-decoupling method (see Table 2.2). The less-than-expected SNR gains at the center are probably due to smaller loop sizes and non-overlapping loops employed in self-decoupling, resulting in lower density of azimuthally distributed coverage. For a given row of azimuthally distributed loops, overlapping the loops would lead to either a larger number of loops (when loop size is equal to the self-decoupled design), or radially larger loops (when loop count is equal to the self-decoupled design). Both cases may produce a stronger receive B1 field in the central regions.

The performance of the $10.5 \mathrm{~T}-32 \mathrm{Rx}$ in $2 \mathrm{D}$ accelerated acquisitions can be compared with the 7T-32Rx in terms of g-factors (presented in Table 3.2). The mean inverse g-factor of the $10.5 \mathrm{~T}-32 \mathrm{Rx}(1 / \mathrm{g}=0.69)$ is $18 \%$ more than mean inverse $\mathrm{g}$-factor of the $7 \mathrm{~T}-32 \mathrm{Rx}$ $(1 / \mathrm{g}=0.59)$ for $4 \times 4$ acceleration. 


\begin{tabular}{|l|lc|lc|}
\hline & \multicolumn{2}{|c|}{$g$-factor } & \multicolumn{2}{c|}{ Inverse $g$-factor } \\
\hline 10.5T-32Rx & Mean & 1.44 & Mean & 0.69 \\
& Max & 1.8 & Min & 0.56 \\
\hline 7T-64Rx & Mean & 1.3 & Mean & 0.77 \\
& Max & 1.9 & Min & 0.53 \\
\hline 7T-32Rx & Mean & 1.7 & Mean & 0.59 \\
& Max & 2.7 & Min & 0.37 \\
\hline
\end{tabular}

Table 3.2: Comparing g-factor values for $4 \times 42 \mathrm{D}$ accelerated acquisitions using 10.5T-32Rx with $\mathrm{g}$-factor values for 7T-64Rx and 7T-32Rx [1].

\subsection{Discussion}

Previous studies have shown that ultimate intrinsic SNR increases with the magnetic field magnitude [27, 41, 65]; the use of high density receive arrays is necessary at UHF to approach ultimate intrinsic SNR [30] available at ultra-high fields and capitalize on the acceleration potential of ultra-high field MRI. However, capturing these potential gains experimentally with increasing number of channels in UHF also depends in the execution of the RF coil array, both in accelerated and unaccelerated imaging. As such, strategies for mitigating interelement coupling and receiver noise play a critical role.

The effort presented here is the first implementation of the self-decoupling method in a 3D conformal, high density 32-channel receive-only array for human brain imaging, albeit in this case it was done for the very high frequency of $447 \mathrm{MHz}$. Our data confirms Yan et al.'s [46] suggestion that in high-density, 3D conformal receive arrays, self-decoupling can be paired with preamplifier decoupling to improve inter-element isolation. Importantly we demonstrate that the combination of higher frequency $(447 \mathrm{MHz})$, smaller loop sizes appropriate for a 32-channel receiver and distributed inductance of such loops being in the range required for self-decoupling, can be exploited to achieve acceptable inter-element isolation with a more uniform capacitive distribution at $447 \mathrm{MHz}$. The resulting approach improves the RSOS of magnitudes of receive fields substantially, and results in better noise- 
correlation-weighted SNR compared to overlapped decoupling.

Experimental noise correlation matrix demonstrated effective inter-element receiver decoupling which is indicative of promising potential for parallel imaging. Indeed, the array provided substantially improved parallel imaging performance at $10.5 \mathrm{~T}$ compared to a $32-$ channel industry standard RF coil operating at 7T, and comparable to a 64-channel array at 7T (Table 3.2). It is not possible to distinguish if the gains are primarily because of the coil design and inter-element decoupling approach employed or reflect the gains expected from going to a very high magnetic field [27, 29, 40]. Nevertheless, the approach employed has enabled the realization of the anticipated gains in parallel imaging performance at UHF that push the electromagnetic operating regime toward the far field domain.

We anticipate that at ultra-high frequencies (447 MHz and higher) the self-decoupling strategy will significantly simplify future high density receive array design and construction as it obviates practical complexities of common decoupling techniques. In addition, the receive array as constructed was shown to result in limited transmit field distortion following insertion into a complex, 16-channel transmitter. This will facilitate the electromagnetic simulation effort for specific absorption rate and regulatory validation by accounting for the small impact of the receive array by including it in a safety margin when calculating the maximal allowable power use. Furthermore, limited receiver-transmitter interaction should enable an interchangeable coil setup where a single transmitter can be used with 32-channel, 64-channel, and 128-channel receivers.

Peripheral and central SNR gains presented here in comparison to 7T confirm the expected gains for ultra-high field imaging targeting the human head [23, 27, 65, 89, 90]; however, consistent quantitative comparisons are difficult to make. In EM simulations, it is generally reported that the gains in ultimate intrinsic SNR are supralinear with field magnitude in the center of the head $[27,65,89]$, all suggesting approximately quadratic increases with field magnitude. In the periphery, however findings are not so consistent; Wiesinger et. 
al. [27] still predict supralinear gains in the periphery but other studies predict linear [65] or even less [89]. In part, the discrepancies may emerge from the particular human head model, or approximations of the human head employed, physical locations of the current distribution relative to the model, and the ROI's over which the ultimate intrinsic SNR was averaged. Irrespective of the quantitative differences, however, the afore mentioned studies predict supralinear gains with field magnitude averaged over the brain, approximately in agreement with $B_{0}^{1.65}$ dependence experimentally reported by Pohmann et. al. [23]. The results from our $7 \mathrm{~T}$ and $10.5 \mathrm{~T}$ comparison agrees with this general conclusion. However, the gains we measured were less than quadratic overall, while they were quadratic in the peripheral ROI. In interpreting these results, it must be realized that different receive array designs can lead to different SNR gain distributions and may have different degree of success in capturing the ultimate intrinsic SNR in different regions of the object being imaged; this will impact comparisons between two different coils at two different field strengths. Moreover, that the implementation of a real coil can deviate significantly from the modeling efforts, especially of ultimate intrinsic SNR. Compared to simulations of ultimate intrinsic SNR, the proximity of the coil elements to the object is not uniform, nor is the size of the coils employed. While we demonstrate that the self-decoupling approach is an improvement in capturing SNR compared to the overlapped-decoupling method when both methods employ equal numbers of geometrically identical loops, an overlapped design allowing for larger loops covering the same surface area may potentially improve central SNR. There are also other potential noise sources, such as imperfect preamplifier decoupling which can lead to the preamplifier noise being propagated back into receive loops.

This work is a significant milestone also towards building 64-channel and 128-channel receiver arrays for human brain imaging at 10.5T. It has turned into the benchmark for comparison of future $10.5 \mathrm{~T}$ receive arrays [91]. It is fully anticipated that scaling up to a 128-channel design will pose additional challenges in noise mitigation, preamplifier design, 
and receiver-transmitter interactions. However, expected SNR and parallel imaging gains provide strong rationale and impetus to address such challenges. 


\section{CHAPTER 4}

64-CHANNEL RECEIVE ARRAY AT 447MHZ

Finally - coil looks fabulous!!

Andrea Grant - Dec 13, 2020.

The successful experience of building the 32-channel receiver was a milestone in efforts towards higher density receive arrays. The next logical step was building a 64-channel receiver, to investigate SNR gains compared to the 32-channel array, especially at the periphery of the brain. Discussing the plausible gains of higher density receivers as a function of channel count, Keil and Wald wrote in 2013, "it remains to be seen that this factor $\left(\sqrt{N_{c h}}\right)$ is enough to overcome the steep drop-off of the smaller surface coils as a function of depth" [32]. The results of this chapter are a step in the direction of resolving their point. Part of the materials in this chapter were published in [2].

\subsection{INTRODUCTION}

As mentioned before, signal-to-noise ratio and parallel imaging advantages of ultra-high field MRI are beneficial to extensive clinical and research applications. Radiofrequency 


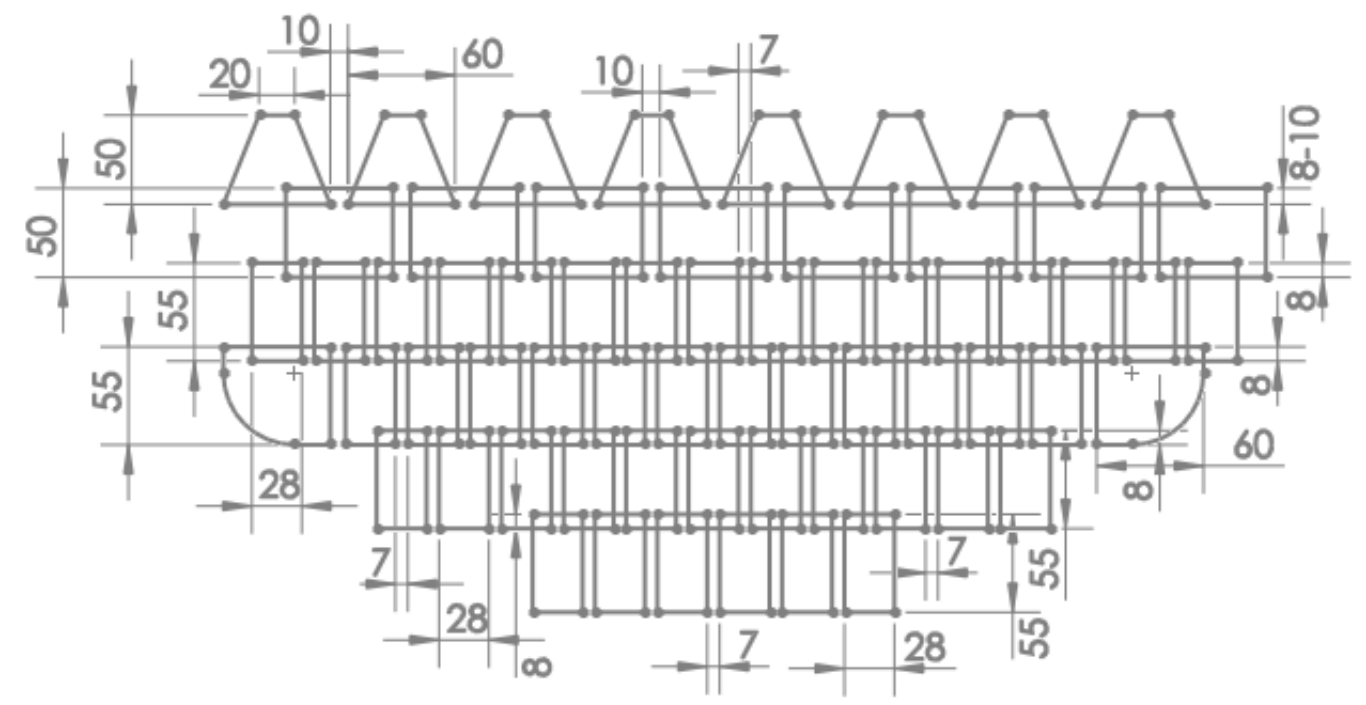

Figure 4.1: Flattened layout of the 64 channel receiver (approximate dimensions in $\mathrm{mm}$ ).

receive arrays are an integral requirement of realizing those advantages.

In my previous works $[3,55]$, I built a $10.5 \mathrm{~T}$ 32-channel receiver $(10.5 \mathrm{~T}-32 \mathrm{Rx})$ and compared it with an industry-standard (Nova Medical Inc. Wilmington, MA) 32-channel array operating at 7T and demonstrated the expected SNR and PI gains at the higher operating frequency of $447 \mathrm{MHz}$. Here, we extend the previous work in several ways, most notably by scaling the principle of self-decoupling to a 64 -channel receiver at $10.5 \mathrm{~T} / 447 \mathrm{MHz}$ for the first time, and present a novel 10.5T 64-channel receive array (10.5T-64Rx) and demonstrate further significant SNR gains compared to our previous receive array (10.5T$32 \mathrm{rx})$.

\subsection{Methods}

The 64 channel receive array was built using self-decoupling [46] between azimuthally distributed elements of each row (there are six rows of loops). While there was overlap between rows along the z-direction ( $B_{0}$ direction), the elements within each row did not use overlap or inductive decoupling. Figure 4.1 shows the coil layout. Preamplifier decou- 
pling $[3,25]$ was used to further improve the receiver array noise correlation. Cable traps were incorporated to suppress shield-current-induced noise on preamplifier input coaxial cables. Figure 4.2 presents the as-built photograph of the 64-channel receive array.

A 16-channel dual row loop transmitter was used in combination with the receive arrays [74] (both the 64 channel and the 32 channel receivers can slide in and out of this 16 channel transmitter). The phantom used in the SNR measurements was the same light-bulb phantom used in the previous chapter (Figure 3.6a).

As discussed in Section 3.4, the preamplifier decoupling of the 32-channel receiver could be improved. In the 32rx, preamplifier decoupling relied on perfect quarter wavelength impedance transformation. This, of course, was challenging in practice as small inaccuracies in coaxial cable length would substantially affect preamplifier decoupling quality. In the 64-channel receiver, the preamplifier board was revised in multiple ways, including the incorporation of a variable capacitor at the preamp input to adjust phase which allowed fine-tuning of preamplifier decoupling ${ }^{1}$. The feed board of the 64rx elements was also revised to incorporate two diodes for improved preamplifier protection and receiver detuning during transmit ${ }^{2,3}$.

Identical protocols were used in both experimental measurements (with the 64 and 32 channel receivers). AFI [85] maps were acquired and SNR acquisitions were approximately fully relaxed $\left(T_{R} \gg T_{1}\right)$ transverse multi-slice GRE sequences. Details of our experimental protocol used to calculate SNR are discussed in Chapter 3 and published previously [3]. It

\footnotetext{
${ }^{1}$ Another practical improvement of the preamplifier board was adding RF chokes and redistributing them on the board to shorten the conductor path that could carry current.

${ }^{2}$ The structural integrity of the 64rx was also improved via several modifications to the former.

${ }^{3}$ As a side note, the logistical challenges of building a 64 channel receive array are also remarkable. Upgrading the interface of the MR scanner to support a 64 channel receive array was one such challenge. Construction complexity affecting the quality of the final coil was another. Learning from the experience of the 32-channel receiver, the preamplifier substrates were redesigned to be dismountable. This allowed for a modular construction where the output cabling of the preamplifiers was built and tested previous to mounting them on the 64-channel receive former. This reduced complexity is important as it allows for better access, extensive testing, and therefore a more reliable coil requiring less maintenance. There are also unresolved debates on design details. For instance, while I used traps on the preamplifier boards at their output to prevent shield currents on output cables, shielding these traps may reduce their interaction.
} 

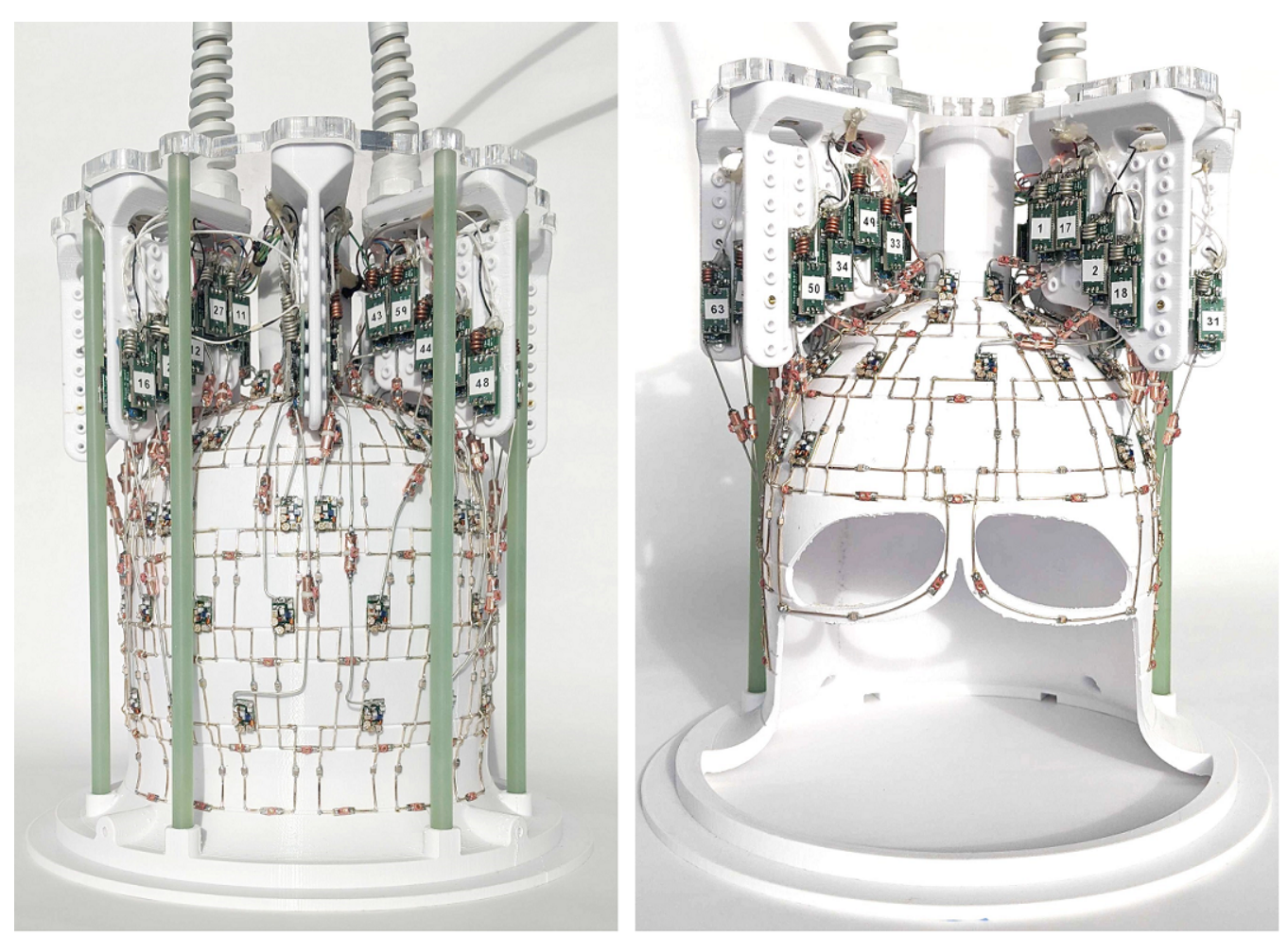

Figure 4.2: As built photograph of the 64 channel 10.5T receive array for human brain imaging.

should be noted that SNR calculations presented here are normalized for flip angle, voxel size, and bandwidth. Since the same MR scanner, field strength and phantom were used in both experiments, noise figure of the receive chain and $T_{2}^{*}$ time constants were considered similar.

\subsection{RESULTS}

Figure 4.3 shows the experimental noise correlation measured at the scanner. Maximum (off diagonal) noise correlation was 0.31 , corroborating the effectiveness of self-decoupling in achieving inter-element isolation. Figure 4.4 presents a comparison between the SNR of the 64 channel receiver and 32 channel receiver over axial slices. The 64 channel receiver provides higher SNR both at the periphery and center (the dark center in the left two slices is an oil reference). Figure 4.5 provides the SNR comparison in the central axial, sagittal 


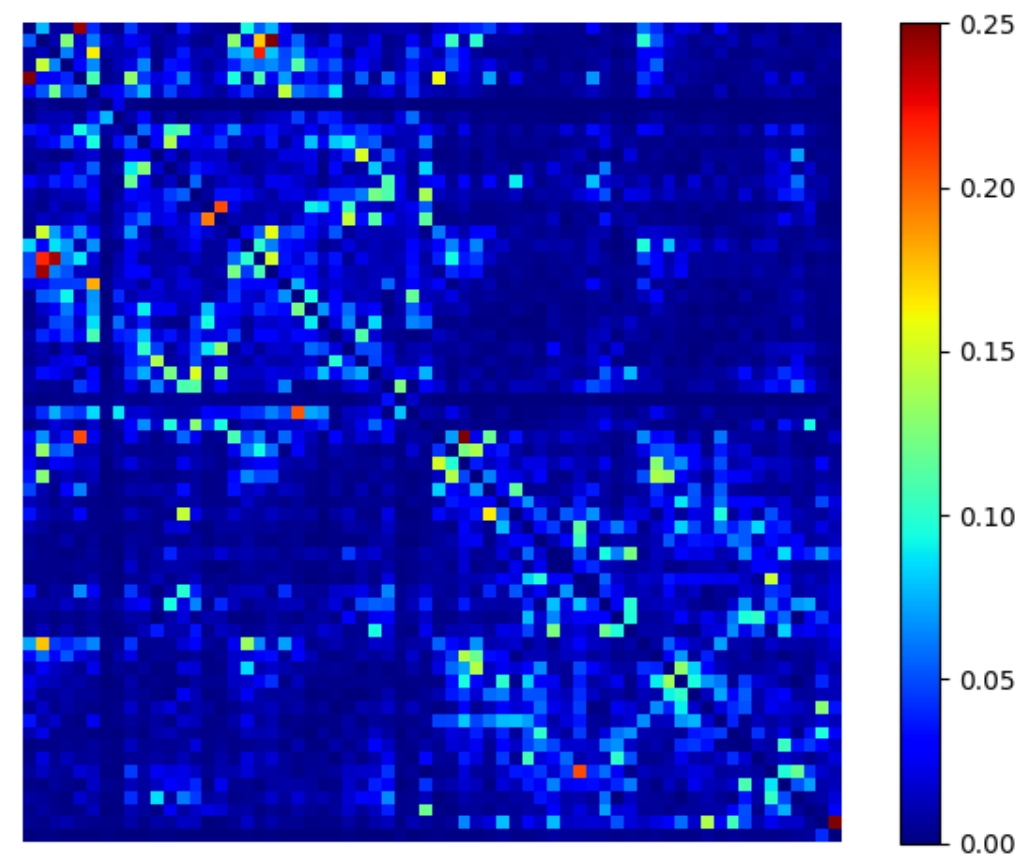

Figure 4.3: Experimental noise correlation matrix of the 64-channel receive array.

and coronal slices of 3D SNR data sets. These figures demonstrate, qualitatively, the gain in SNR using the higher channel count receiver. While the gain in the periphery is most noticeable, the SNR in the central ROI has also improved.

Table 4.1 presents a quantitative comparison of SNR averaged over the entire phantom for various values of $d$, depth into the phantom measured from the surface, and delineates the boundary between peripheral and central ROIs as shown in Figure 3.6b. The 64-channel receiver provides 1.95 times more overall SNR on average, with 2.41x and 1.55x SNR gains at the peripheral and central ROIs (when the boundary between the peripheral and central ROIs is at $20 \mathrm{~mm}$ from the phantom surface).

\subsection{Discussion}

We present the successful design and construction of a 64-channel receive array for human brain imaging at $10.5 \mathrm{~T}$. The receive array takes advantage of preamplifier and self- 


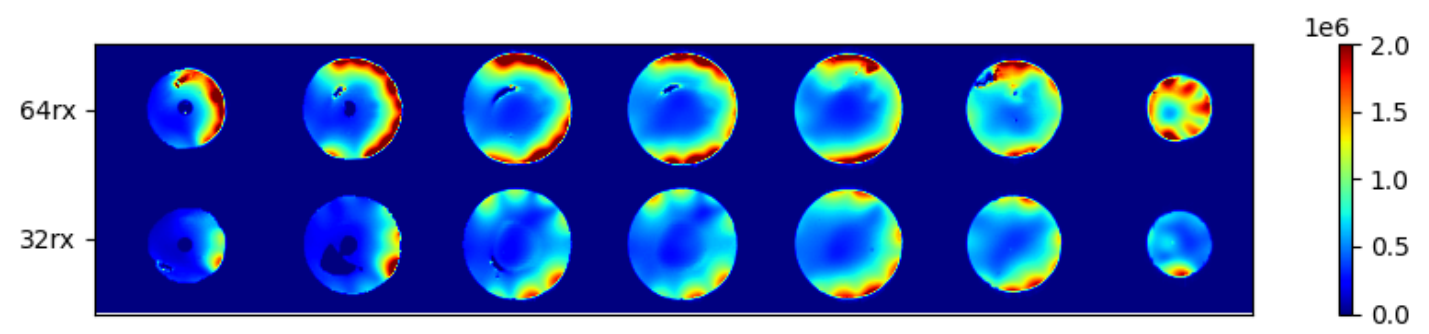

Figure 4.4: Experimental SNR comparison between the 64-channel 10.5T receive array (top row) and the 32-channel $10.5 \mathrm{~T}$ receive array (bottom row) at 7 axial slices.

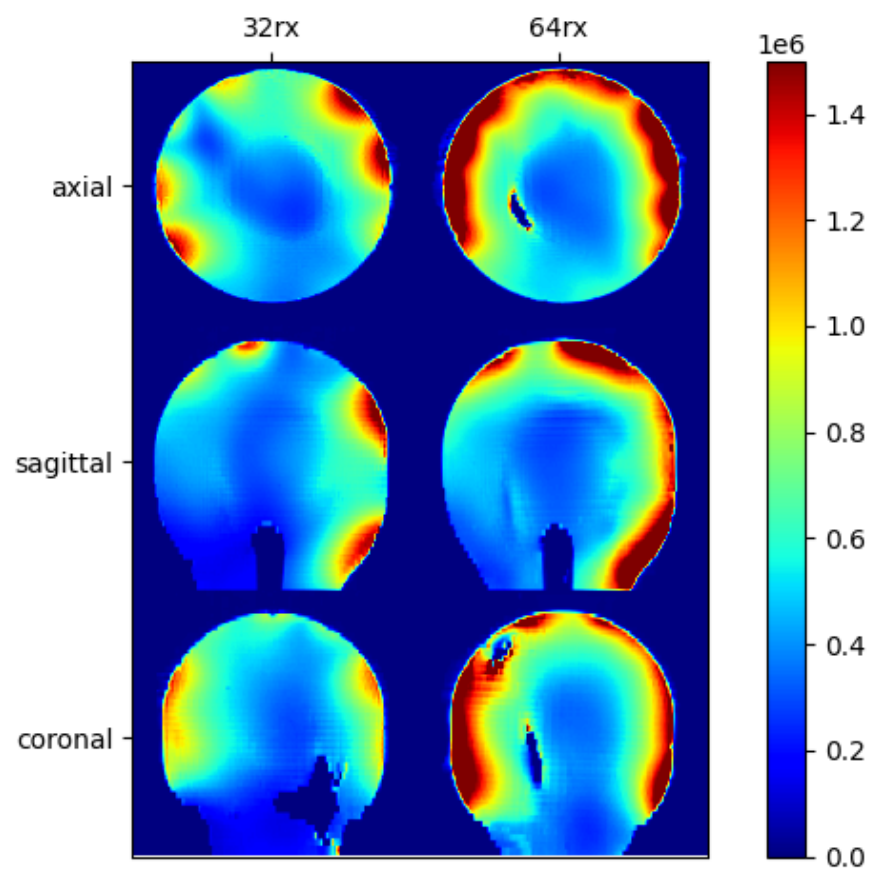

Figure 4.5: 3D SNR comparison between the 64-channel 10.5T receive array (right column) and 32-channel 10.5T receive array (left column) in the central axial, sagittal, and coronal slices.

\begin{tabular}{|c|c|c|c|}
\hline Depth (d, mm) & Peripheral SNR ratio & Central SNR ratio & $\begin{array}{c}\text { Overall Average } \\
\text { SNR ratio }\end{array}$ \\
\hline 30 & 2.18 & 1.51 & 1.95 \\
\hline 25 & 2.28 & 1.52 & 1.95 \\
\hline 20 & 2.41 & 1.55 & 1.95 \\
\hline 15 & 2.59 & 1.61 & 1.95 \\
\hline 10 & 2.62 & 1.76 & 1.95 \\
\hline
\end{tabular}

Table 4.1: Experimental SNR ratios (average 64-channel snr divided by 32-channel snr). Depth $d$ marks the boundary between the peripheral and central ROIs and measures the width of the peripheral ROI from the phantom surface. 
decoupling principles and lays the groundwork for our future higher density receivers by mitigating the requirement of overlap or inductive decoupling. Whereas overlap/inductive receive array decoupling could be practically prohibitive for building $100+$ channel receive arrays, our progress with self-decoupling receive arrays is an important development in that direction. The lessons learnt from the successful implementation of the 64 channel receiver have informed the design of CMRR's 128 channel receiver for human brain imaging at $10.5 \mathrm{~T}$.

Looking back at Keil and Wald's question mentioned at the start of this chapter, our results show over 1.5 times SNR gain at the central ROI (Table 4.1), using the 64-channel receiver compared to the 32-channel receiver. First, looking at Figure 3 from Keil's paper [32], it should be noted that they attributed their reported SNR gains to "tighter fitting geometries" of higher density receivers. In contrast, the two receive arrays compared in this chapter are built on identical formers (i.e. have identical geometries); therefore, SNR gains reported here cannot be explained by variations in coil-to-sample distance. Second, the two coils presented here employ similar layouts (with self-decoupling and overlap); so coil design variations (which often make coil comparisons complicated) are not a contributing factor here. Given that the elements of the 64rx are indeed significantly smaller than the $32 \mathrm{rx}$ elements, and that the average central gain of 1.51 is more than $\sqrt{64 / 32}$, it seems that the $\sqrt{N_{c h}}$ factor can in practice overcome the steep SNR drop-off of smaller loop elements.

While the maximum noise correlation of CMRR's existing 7T-64rx is 0.67 [1], the maximum noise correlation of the $10.5 \mathrm{~T}-64 \mathrm{rx}$ presented here is 0.31 , both measured with a noise scan at the MR scanner. Given the preamplifier noise increases linearly with the field strength (and for a host of other reasons), decoupling is generally more challenging at higher frequencies (see for example [32, 54]). The more challenging yet reduced noise correlation can be attributed to design improvements, including the use of self-decoupling instead of overlap. The 7T-64rx has on-coil preamplifiers - with preamplifier output cables 
passing along the input of other preamplifiers, potentially contributing to noise correlation - whereas the 10.5T-64rx has off-coil preamplifiers with careful cable routing and traps aimed at mitigating noise correlation.

There exist synergies between ultrahigh fields and high density receive arrays (specifically in terms of the parallel imaging and SNR gains at ultra-high field and the role of high density receivers in achieving these gains, see Chapter 1 and, for instance, discussion in Ugurbil et al. [1] and references therein). In agreement with this synergy, our results confirm previous expectations of SNR gains using higher density receivers at ultra-high fields. Previous simulations had indicated that using higher density of receive channels improves the SNR and would be especially advantageous in the peripheral regions of the brain. Our results also demonstrate a more substantial SNR gain at the periphery versus the central ROIs. 
CHAPTER 5

_GANS FOR PARALLEL IMAGE RECONSTRUCTION

The goals we should be pursuing aren't immediately evident to us.

Michael L. Littman

While the previous chapters pursued SNR gains using better hardware, this chapter is attempting the same objective from the reconstruction front. As discussed in Chapter 1, parallel imaging techniques (empowered by the high density receive arrays mentioned so far) come at the expense of noise amplification. Here we use a data-driven deep learning method for reconstruction of undersampled acquisitions to mitigate noise amplification. The materials in this chapter were published in [92].

\subsection{INTRODUCTION}

MRI is a prevalent non-invasive medical imaging technique with various clinical and research applications. A major advantage of MRI is its potentially high resolution; however, MRI generally requires lengthy acquisition times to achieve high resolution images. Undersampling the MR signal (obtained in frequency domain a.k.a. $k$-space) is a method to 
accelerate such time-consuming acquisitions. Parallel imaging refers to the methods used for reconstructing MR images from undersampled $k$-space signal. Generally, parallel image reconstruction techniques take advantage of the additional encoding information obtained using (ideally independent) elements of a receiver array and/or mathematical properties of the frequency domain signal to compensate for the loss of information due to the undersampling. Nevertheless, consequences of that information loss generally detract from the quality of the images reconstructed from undersampled $k$-space.

The aim of improving the undersampled reconstructions can be pursued from multiple different angles. While an extensive review of all such research efforts is beyond the scope of this article, we still mention a few relevant works in each line of research to provide context for the current paper. In terms of hardware, there has been significant effort in the MR research community to improve the sensors used to acquire the signal (radio-frequency coils) to reduce noise and noise correlation between different channels or to take advantage of additional receive channels (e.g. [3, 32, 50, 51, 74]). There has been a wider variety of advancements in the post-processing front. SENSE [35] and GRAPPA [36] are two of the primary methods for parallel MR image reconstruction. GRAPPA tries to estimate the missing $k$-space signal but it inherently suffers from noise-amplification. Generally, the $k$-space undersampling comes at the expense of aliasing in reconstruction. Several variations and extensions to SENSE and GRAPPA have been proposed which primarily rely on regularization to suppress noise-amplification. Compressed-sensing also relies on non-linear optimization of randomly undersampled $k$-space data, assuming the data is compressible [42]. Compressed sensing MRI generally utilizes total variation, wavelet/cosine transforms, or dictionary learning as sparse representations of the naturally compressible MR images.

More recently, side effects of existing techniques (noise amplification, staircase artifacts of total variation, block artifacts of wavelets, relatively long reconstruction time of 
iterative optimization techniques, etc) and the advent of public MR image datasets have encouraged researchers to look into deep learning techniques which have often outperformed conventional regularization and/or optimization-based techniques in various applications, including variants of the undersampled image reconstruction problem (e.g. [43, 93]). Among the promising literature, several works have used generative adversarial networks [44, 94] to reconstruct undersampled images. Yang et al. [95] proposed a GAN to address the aliasing artifact resulting from the sub-Nyquist sampling rate. Their proposed architecture used a pretrained network to extract an abstract feature representation from the reconstruction and enforce consistency with the target in that feature level. Murugesan et al. [96] and Emami et al. [97] used context dependent/attention-guided GAN which has a feedback loop back to the generator input providing information focusing on local deviations from tissue. Mardani et al. [98] and Deora et al. [99] used residual skip connections inside each convolutional block of their generator. It is noteworthy that Mardani suggests the discriminator outputs can be used to focus on sensitive anatomies. Dar et al. [100] also used perceptual priors in their multi-contrast reconstruction GAN. The above mentioned studies using GANs have demonstrated enhanced performance compared to state of the art compressed sensing and other parallel imaging reconstruction techniques. However, one of the primary critiques of GAN-based reconstruction is the suggestion that GANs are prone to hallucination (see for example [98]).

Here, we propose a novel method for reconstruction of undersampled/accelerated MRI images that combines GRAPPA and GAN to further improve the reconstruction quality by building on our proof-of-principle demonstration [101]. Our primary contributions include:

- we propose a combination of GRAPPA and GAN to address noise amplification,

- in addition to the adversarial losses, we include data-consistency and perceptual feature level loss for artifact removal. 

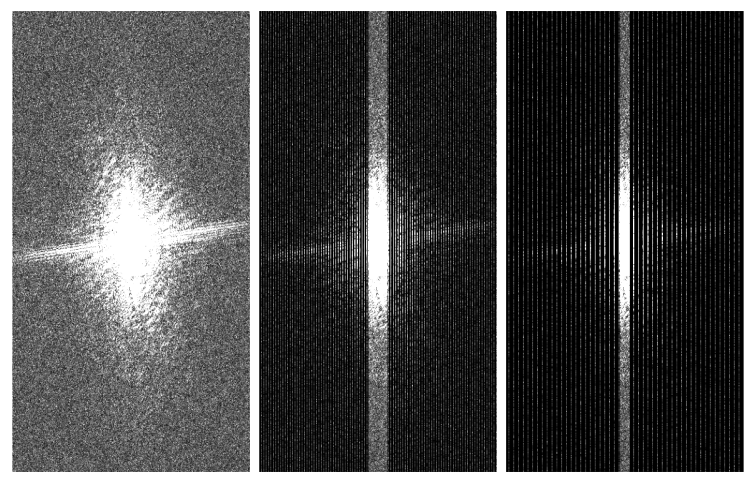

Figure 5.1: Equidistant $k$-space undersampling with random position of the first $k$-space line, keeping the central $k$-space fully-sampled (ACS lines used for GRAPPA). From left to right: fully sampled, subsampled with $\mathrm{R}=4$, subsampled with $\mathrm{R}=8$.

\subsection{Methods}

\subsubsection{Undersampling scheme}

The original data is fully sampled in $k$-space, allowing for comparison of undersampled reconstructions with a fully-sampled ground truth reconstruction. Various undersampling schemes have been used in the literature, with uniform random subsampling, equidistant random subsampling, and Gaussian random subsampling being the primary schemes. Given that our dataset (discussed in more detail shortly) is composed of 2D axial slices, our analysis uses only 1D subsampling along the phase encoding direction. Here, we have used equidistant random subsampling while maintaining a fraction of the $k$-space lines at the center of the $k$-space fully-sampled, as is customary in the MRI literature and required for GRAPPA reconstruction. Equidistant random undersampling means that while the $k$-space is subsampled equidistantly, the location of the first $k$-space line is selected at random. For an acceleration rate (or subsampling ratio) of $\mathrm{R}=4,8 \%$ of $k$-space lines were preserved at the center and for $\mathrm{R}=8,4 \%$ of the $k$-space lines were preserved at the center. Figure 5.1 demonstrates the subsampling scheme in $k$-space. 


\subsubsection{Reconstruction method}

Details of GRAPPA implementations have been included in various publications [36]. Briefly, GRAPPA uses linear shift-invariant convolutions in k-space. Convolutional kernels were learned from a fully sampled subset at the center of k-space (auto-calibration signal or ACS lines) constrained by a Tikhonov regularization term and then used to interpolate skipped k-space lines using multi-channel (receive array) raw data. We did a GRAPPA root-sum-of-squares reconstruction of the undersampled, multi channel ${ }^{1}$ image prior to feeding it to the GAN. In a generic GAN, a generator network $\left(G: m_{-} \rightarrow \hat{m}\right)$ competes with a discriminator $(D: \hat{m} \rightarrow(0,1))$ in a min-max optimization problem, $\min _{\theta_{G}} \max _{\theta_{D}} \mathcal{L}\left(\theta_{D}, \theta_{G}\right)=\mathrm{E}[\log D(\hat{m})]+\mathrm{E}\left[\log \left(1-D\left(G\left(m_{-}\right)\right)\right)\right]$,

where the generator learns the mapping from the GRAPPA reconstruction of the undersampled image, $m_{-}$, to its prediction, $\hat{m}$, of the target, fully sampled image, $m$. Note that the GAN is learning in image domain (not the frequency domain).

In essence, first, regularized GRAPPA is used to fill-in the missing $k$-space lines. Then, 2D discrete fast Fourier transform is performed to reconstruct individual images of individual coils. A root-sum-of-squares reconstruction, $m_{-}$, of the individual magnitude images from individual coils is then used as the input to the generator. The generator learns to predict the ground-truth given this subsampled reconstruction while the discriminator learns to classify / distinguish between generator-reconstructed images and ground-truth images.

The GAN was composed of a generator (a UNET [102]) and a discriminator (a convolutional neural network used as a binary classifier). The network architecture is depicted symbolically in Figure 5.2. The UNET consisted of an encoder and a decoder. The encoder was composed of blocks of batch normalization [103], 2D convolution, and leakyReLu, interleaved by max pooling to down-sample the images. Each one of these blocks had

\footnotetext{
${ }^{1}$ The term "channel" in the MRI context refers to the number of sensors or coils used in image acquisition, whereas in the deep learning context, it is used interchangeably with number of kernels or filters.
} 


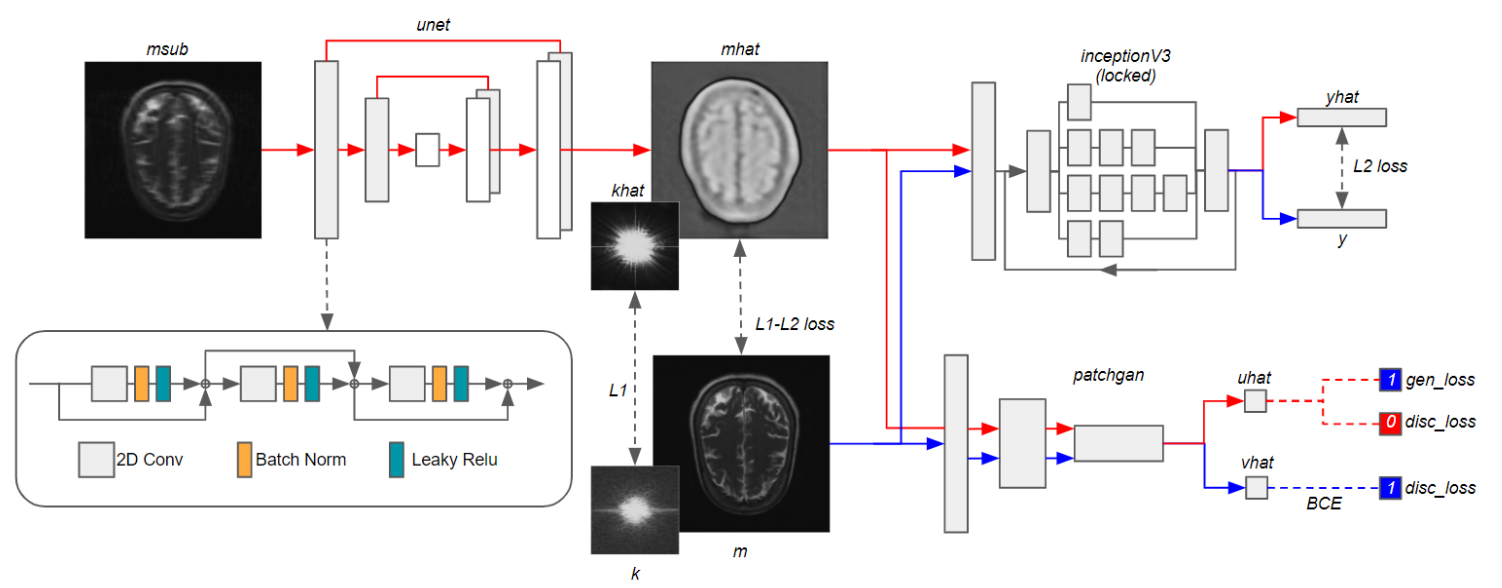

Figure 5.2: Symbolic network architecture. The UNET consisted of five levels, starting with 64 channels at the first layer. Kernel size used for $2 \mathrm{D}$ convolutions was $3 \times 3$ (in both $\mathrm{R}=4$ and $\mathrm{R}=8$ experiments, due to computational limitations). The InceptionV3 network was pretrained on ImageNet and used to extract and compare features from the generator output and target image. Each convolution block of the UNET consisted of three layers of convolution, batch normalization, leakyRelu interleaved with resnet-type skip connections.

three convolutional/activation layers with in-block (resnet type) skip connections passing the information derived at earlier layers to the features computed at later layers. The decoder was composed of similar normalization, convolution, leakyReLu blocks interleaved by transpose 2D convolutions for up-sampling. Skip connections were used to add highlevel feature representations of the encoding path to elements of the decoding path. The original implementation in [102] learns a prediction of the image, however, we included a skip connection from the input of the encoder to be added to the output of the decoder, so that the UNET is learning the residual (difference). Residual learning (compared to learning the full reconstruction task) proved to be a less challenging task, requiring less model complexity. Furthermore, the addition of the in-block skips noticeably improved performance results. Depth of the UNET was five levels, with the top level limited to 64 kernels at most (due to hardware limitations) and $3 \times 3$ convolutional kernels.

The discriminator was topped with a dense layer and sigmoid activation appropriate for the binary classification of images (classifying generator reconstructions versus ground 
truth) using binary cross entropy loss. In addition to the typical generator GAN loss (binary cross entropy of the discriminator judgment of generator output compared with ones, or $-\log [D(\hat{m})])$, the generator loss was conditioned on a weighted sum of L1 and L2 loss terms comparing generator output with target reconstruction, a data-consistency loss term comparing the output and ground truth in spatial frequency domain (k-space), and an inception loss, comparing the InceptionV3 [104] feature representation of generator output and ground truth. Overall, this results in,

$$
\begin{aligned}
\mathcal{L}\left(\theta_{G}\right)= & \log (D(\hat{m}))+\lambda_{1} L_{1}(\hat{m}, m)+\lambda_{2} L_{2}(\hat{m}, m)+ \\
& \lambda_{D C} L_{1}(\mathcal{F}(\hat{m}), \mathcal{F}(m))+\lambda_{f} L_{2}(\mathcal{I}(\hat{m}), \mathcal{I}(m))
\end{aligned}
$$

where $\mathcal{F}$ is the Fourier transform that maps the images to frequency domain, and $\mathcal{I}$ is the Inception network used to extract features. Note that the Inception network was pretrained on ImageNet [105] and locked (no weight updates) during training. In other words, the InceptionV3 network was used only to calculate a perceptual loss [106], that is to evaluate the performance of the generator (or to accentuate feature level irregularities of generator reconstruction), not as part of the generator's architecture, and need not be used in deployment. In the absence of the Inception feature loss, the L1-L2 loss would focus on pixel level similarity, which is useful in improving the performance metrics (discussed shortly), but leaves noticeable residual aliasing artifacts in the reconstruction. The focus on feature loss (at later epochs of training) helped resolve these residual aliasing artifacts. The addition of the frequency domain data consistency loss helped capture the higher spatial frequency details of the anatomy.

\subsubsection{Dataset}

The data used in this work were obtained from the NYU fastMRI Initiative database, with detailed descriptions of the datasets published previously in $[37,38]$. In the present study, 
we used multi-coil, multi-slice human brain images from the fastMRI dataset. As this dataset includes a variety of real-world acquisitions (with different MR scanners, protocols, artifacts, contrasts, radio-frequency coils, etc) and because variation in each of these factors (especially the number of coils) would cause significant variation in the results, we selected a subset of the dataset limited to images acquired with 16 receive coils ${ }^{2}$. This removed a parameter that would otherwise significantly affect variance in results and therefore, made result interpretation more straightforward. Other than number of coils, and ensuring no subject overlap between train/validation/test sets, no other constraint was imposed on the multi-coil human dataset. The original data were fully sampled. The accelerations (subsampling) were imposed as post-processing steps.

\subsubsection{Evaluation metrics}

Peak Signal-to-Noise Ratio (PSNR) and Structural Similarity (SSIM) were used to assess the performance [107]. The reconstructions were compared with a ground truth, defined as root-sum-of-squares reconstruction of fully sampled k-space data from individual channels. PSNR was calculated as $-20 \log 10(R M S E / L)$ where RMSE is the root-mean-square

error and $\mathrm{L}$ is the dynamic range. SSIM was calculated as $\frac{\left(2 \mu_{x} \mu_{y}+c_{1}\right)\left(2 \sigma_{x y}+c_{2}\right)}{\left(\mu_{x}^{2}+\mu_{y}^{2}+c_{1}\right)\left(\sigma_{x}^{2}+\sigma_{y}^{2}+c_{2}\right)}$ using an $11 \times 11$ Gaussian filter of width 1.5 and $c_{1}, c_{2}$ of $0.01,0.03$ respectively.

\subsubsection{Training and implementation details}

Individual loss terms were normalized to be on similar scales. Training started with a focus on L1 similarity, with $\lambda_{1}=120, \lambda_{2}=30, \lambda_{D C}=0, \lambda_{f}=0$. Midway through training (30 to 50 epochs), the weight balance of L1-L2 loss gradually changed to $\lambda_{1}=30, \lambda_{2}=$ 120. After 100 epochs, the focus shifted to feature loss and data consistency loss while maintaining the L1-L2 weights, with $\lambda_{D C}=30, \lambda_{f}=100$.

\footnotetext{
${ }^{2}$ The choice of "16" was because it was the largest subset of the dataset and it was appropriate for an acceleration factor of $\mathrm{R}=4$, and still reasonable for $\mathrm{R}=8$.
} 
Table 5.1: Comparing average performance results for different 1D acceleration factors (R) with regularized GRAPPA and GAN.

\begin{tabular}{|c|c|c|c|c|}
\hline \multirow{2}{*}{} & \multicolumn{2}{|c|}{$\mathrm{R}=4$} & \multicolumn{2}{c|}{$\mathrm{R}=8$} \\
\cline { 2 - 5 } & PSNR & SSIM & PSNR & SSIM \\
\hline GRAPPA & 33.88 & 0.84 & 22.45 & 0.51 \\
\hline GAN & 37.65 & 0.93 & 29.64 & 0.84 \\
\hline
\end{tabular}

The GAN was trained using 100 subjects (1600 axial slices) while the validation and test dataset each included an additional 100 subjects, without any subject overlap between the three subsets. An Adam optimizer [108] with a customized learning rate schedule was used. Custom python scripts were used for GRAPPA and GAN implementations, with the GAN implemented using TensorFlow 2.2 / Keras. The network was trained for 200 epochs using one NVIDIA Tesla V100 GPU.

\subsection{RESULTS}

Figure 5.3 and Figure 5.4 present a qualitative comparison between reconstructions using regularized GRAPPA and GPGAN. As presented in Table 5.1, with an acceleration factor of $\mathrm{R}=4$, regularized GRAPPA resulted in PSNR $=33.88 \mathrm{~dB}$ and $\mathrm{SSIM}=0.84$. The GAN improved the results to $\mathrm{PSNR}=37.65 \mathrm{~dB}$ and $\mathrm{SSIM}=0.93$. The average root-mean-square error reduced from 0.021 to 0.013 for $\mathrm{R}=4$ and from 0.075 to 0.033 for $\mathrm{R}=8$, using GRAPPA and GAN, respectively. The increase in SSIM is due to reduced standard deviation $\left(\sigma_{x}\right)$ of the GAN reconstruction, suggesting a higher statistical signal-to-noise ratio (SNR $\propto$ mean( signal) / std(noise)) using GAN.

\subsection{Discussion}

While the primary purpose of the proposed technique is reconstruction of sub-sampled kspace (i.e. addressing the aliasing artifact), the fully sampled dataset was contaminated with 

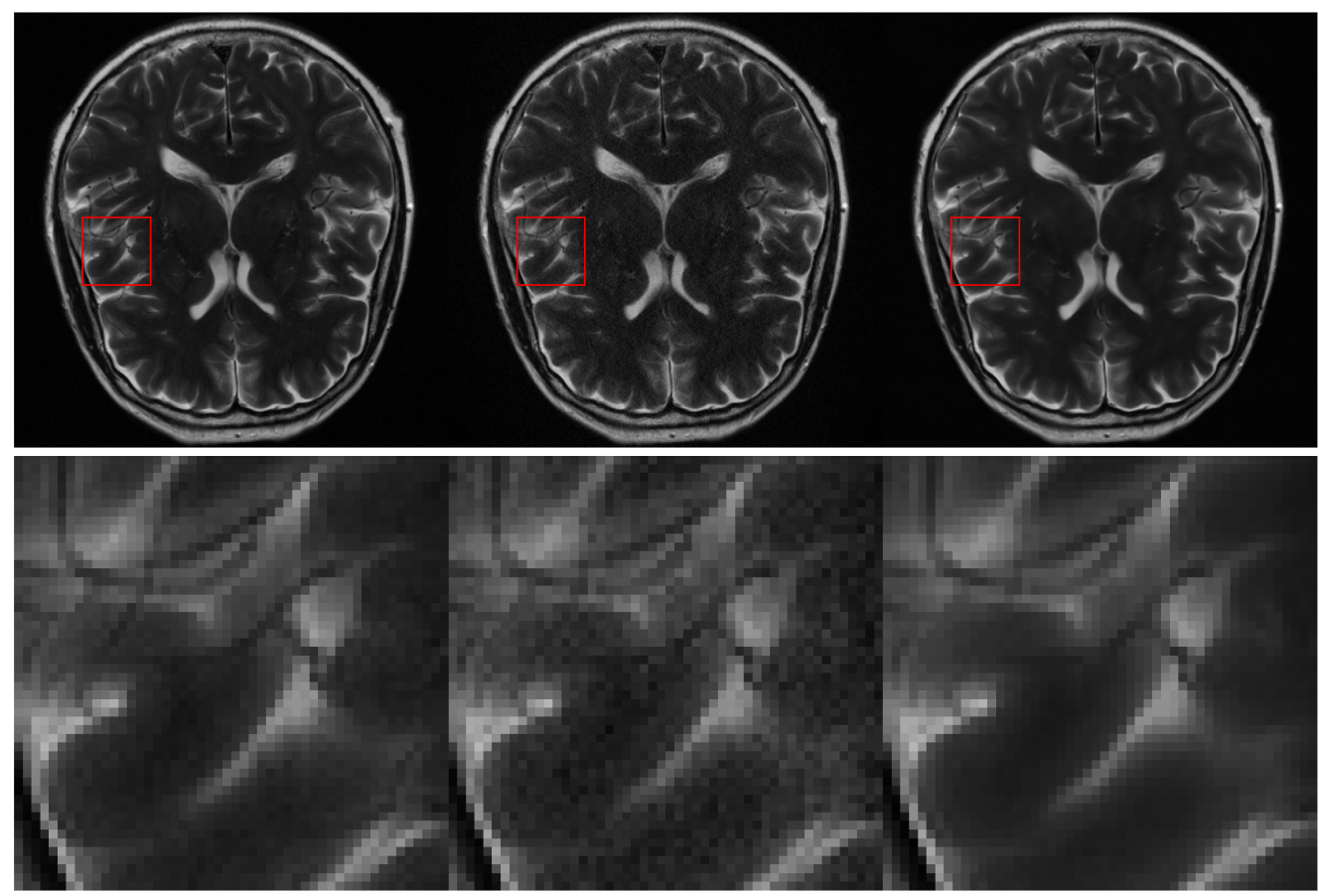

Figure 5.3: Comparing reconstruction quality at acceleration factor $\mathrm{R}=4$. Left: ground truth (fully sampled root-sum-of-squares reconstruction); center: regularized GRAPPA reconstruction (uniform undersampling, 8\% ACS lines); right: GAN reconstruction. 

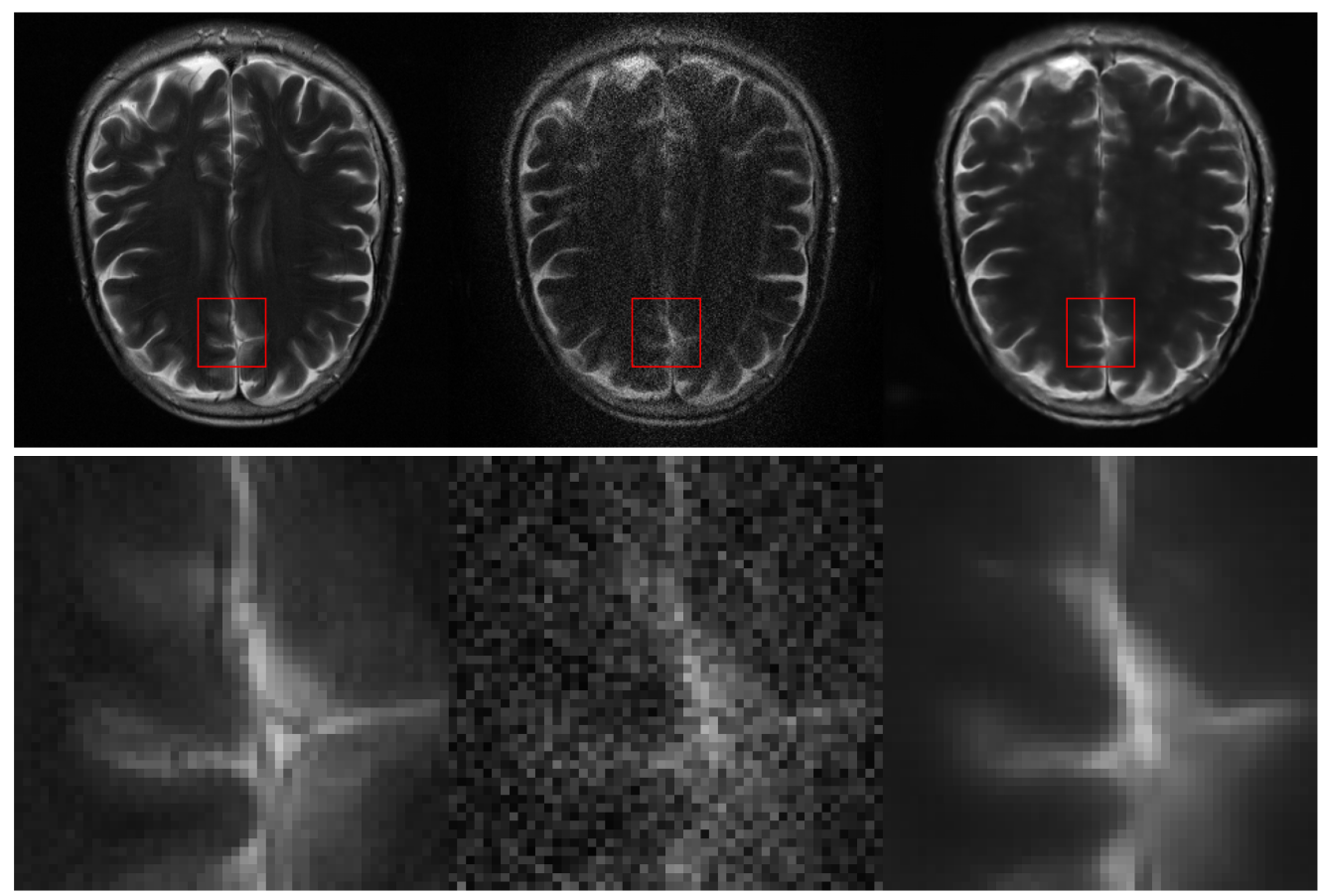

Figure 5.4: Comparing reconstruction quality at acceleration factor $\mathrm{R}=8$. Left: ground truth (fully sampled root-sum-of-squares reconstruction); center: regularized GRAPPA reconstruction (uniform undersampling, 4\% ACS lines); right: GAN reconstruction. 
other common real-world artifacts (Gibbs artifacts, motion artifacts, etc.) which were often mitigated in the final GAN reconstruction. Figure 5.5 demonstrates artifact suppression. Moreover, the GAN reconstruction was effective in denoising reconstructions and improving the average statistical signal-to-noise ratio of the images. Incorporating GRAPPA into the data-driven reconstruction pipeline improves the structural fidelity of the reconstructed images, making sure that no significant structures are added or deleted in the final result (although some details are inevitably lost due to undersampling).

While the dataset included acquisitions using various numbers of receiver channels (from 8 to 23 receive channels), in order to prevent high variance in accelerated reconstructions due to variance in receiver channel count, we used only a subset of the dataset including only acquisitions with exactly 16 receive channels. Nevertheless, an acceleration factor of $\mathrm{R}=8$ using only 16 receive channels results in significant noise in the GRAPPA reconstruction. By comparison, the GAN reconstructions are noticeably less noisy even with $\mathrm{R}=8$ acceleration.

Various elements of the generator loss function ensure different aspects of the reconstruction fidelity. The perceptual prior imposed using the inception network is aimed to achieve feature level consistency. This ensures that prominent features of the reconstruction follow the same distribution as the target dataset. While this helps eliminate the residual aliasing artifacts, it also captures and tries to replicate other real-world artifacts of the target dataset. The latter is mitigated by the data consistency loss term.

In future, we would like to build upon this work by integrating a GAN with a compressed sensing solution of the image reconstruction problem. 

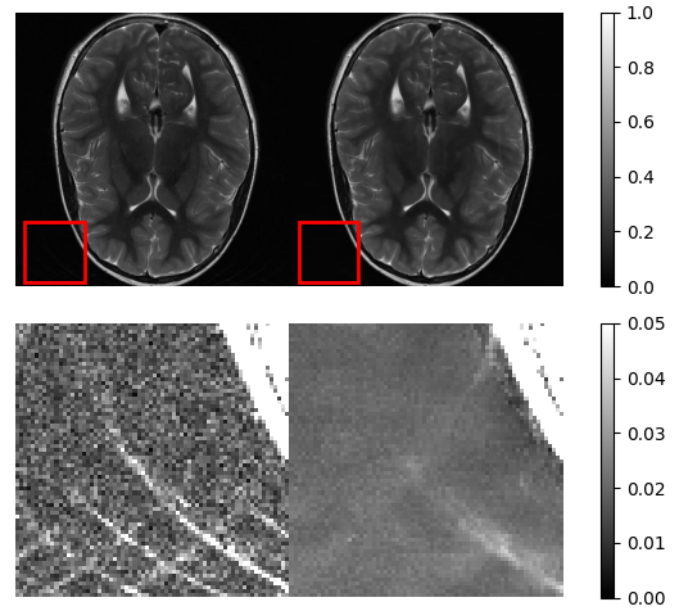

(a)
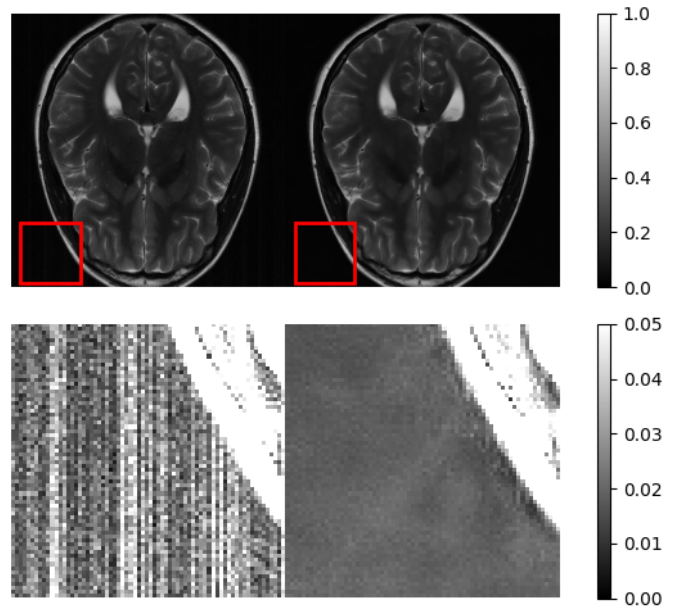

(b)

Figure 5.5: Denoising and artifact suppression using the proposed GAN. In both (a) and (b), the left subfigures are the ground truth and the right subfigures are the GAN reconstructions. The lower row are the zoomed-in and rescaled detail view of the respective red boxes. 
CHAPTER 6

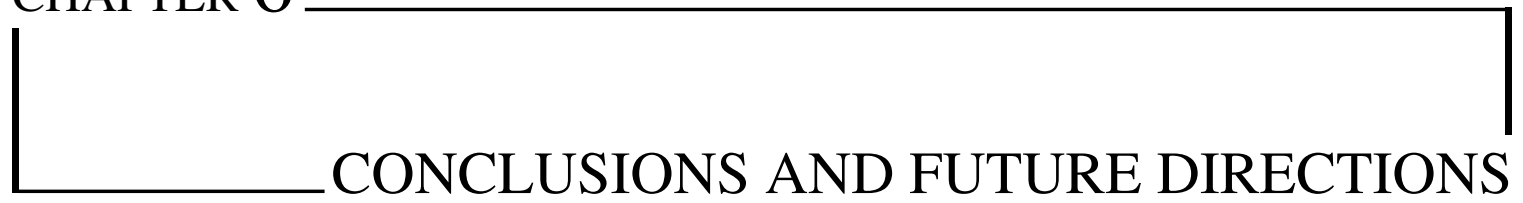

I am proud and excited about all you achieved in the previous years.

Gregor Adriany - Feb 24, 2021.

Loop-based receive-only arrays have become an integral part of magnetic resonance imaging as they provide significant advantages in cortical and central signal-to-noise-ratio and parallel imaging performance. Chapters 2-4 of the thesis dealt with hardware solutions that enable realization of these ultra-high field MR advantages. Chapter 5 was an effort to address the noise amplification characteristic of the parallel imaging reconstruction technique GRAPPA.

\subsection{SELF-DECOUPLING}

Conventional techniques to mitigate noise correlation between receive-only elements require geometric overlap, inductive / capacitive / preamplifier decoupling, or dramatically unbalanced impedance distribution. Here, I presented a balanced self-decoupling method specific to receive-only arrays at $10.5 \mathrm{~T} / 447 \mathrm{MHz}$. Compared with overlap decoupling, the 
proposed self-decoupling method results in improved SNR while providing similar interelement isolation. Furthermore, self-decoupling reduces the implementation complexity, paving the way for high density receive arrays.

\subsection{2-ChANNEL RECEIVER}

The self-decoupling technique was exploited to build the first self-decoupled 32-channel receive array $(32 \mathrm{Rx})$ for human brain imaging at $10.5 \mathrm{~T}$. The $10.5 \mathrm{~T}-32 \mathrm{Rx}$ provided 2.25 times more peripheral and 1.47 times more central SNR compared to an industry-standard 7T-32Rx, with an overall average of 1.81 times SNR gain, in unaccelerated acquisitions. In 2D 4x4 accelerated acquisitions, the mean inverse $g$-factor of the $10.5 \mathrm{~T}-32 \mathrm{Rx}$ was $18 \%$ more than the mean inverse $g$-factor of the 7T-32Rx. This achievement delivered the muchanticipated SNR boost in highly accelerated ultra-high field imaging required for further understanding of human brain function and connectivity.

\subsection{4-CHANNEL RECEIVER}

Building on the successful development of self-decoupling-based UHF array design principles, a novel self-decoupled 64-channel receive array was built for human brain imaging and compared to the 32-channel receive array at $10.5 \mathrm{~T} / 447 \mathrm{MHz}$. Maximum noise correlation of the $10.5 \mathrm{~T}$ 64-channel receiver was 0.31 measured experimentally at the MR scanner which is an improvement compared with maximum noise correlation of 0.67 of the 7T 64-channel receiver. SNR comparisons showed 1.95 times more SNR averaged over the sample relative to the 32-channel array at 10.5T, with 2.69 times peripheral and 1.61 times central SNR gain (when the peripheral versus central boundary is at $20 \mathrm{~mm}$ from the phantom surface). The further-improved ultra-high field SNR is primarily attributed to the higher density of the 64-channel receive array, confirming the anticipation that increased 
channel count can (more than) compensate for the intensified SNR drop-off (as a function of depth into tissue) of smaller loops. These results set the stage for further SNR gains at $10.5 \mathrm{~T}$ by building a 128 -channel receive array.

\subsection{GANS FOR PI RECONSTRUCTION}

Parallel imaging reconstruction techniques, including GRAPPA and its variants which are utilized extensively in clinical and research settings, reduce image acquisition time via $k$-space undersampling at the expense of amplified noise. To improve the SNR of reconstructed images, a reconstruction model combining GRAPPA with a conditional generative adversarial network was developed and tested on multi-coil human brain images from the fastMRI dataset. For various acceleration rates, GAN and GRAPPA reconstructions were compared in terms of PSNR and SSIM. For an acceleration rate of R=4, PSNR improved from 33.88 using regularized GRAPPA to 37.65 using GAN while mean SSIM improved from 0.84 to 0.93 . GAN consistently outperformed GRAPPA for various acceleration rates. Furthermore, it was shown that structural noise artifacts can be mitigated using the GAN.

\subsection{FUTURE DiRECTIONS}

The anticipated FDA approval of the transmitters to be used for human brain imaging at 10.5T will allow for in-vivo experiments using the receive arrays presented here. In this context, these receivers are expected to contribute to various future human brain research studies in CMRR.

The self-decoupling principle developed here to build $10.5 \mathrm{~T} / 447 \mathrm{MHz}$ receiver loop arrays is applicable to design, construction, and validation of loop-based transmitter or transceiver arrays as well. At ultra-high field, it has been prohibitively challenging to replicate in simulation the experimental magnetic field of transmitters that use alternative decou- 
pling techniques (such as overlap and transformer decoupling) with the level of accuracy necessary for safety validations. These challenges, at least in part, stem from the deviations of manual implementation of decoupling transformers from designs and simulations. Going forward, designing transmitter arrays with self-decoupling will simplify implementation, obviate practical deviations and facilitate the safety validation efforts [109].

There are early indications in simulations [110-112] that SNR of high density receive arrays may benefit from shielding the RF coils (at a specific shield diameter) or using high permittivity materials (with specific dielectric properties) in the construction of receiver formers. These findings, if replicated experimentally, can be a promising avenue for further improvement of SNR at ultra-high field. One challenge in implementing these ideas is that the optimum shield diameter or permittivity seem to be a complex function of several factors, including field strength and, more intricately, the particular RF coil design. This would mean that determining the optimum shield diameter or permittivity can be very challenging, especially given the difficulty of replicating in simulations the deviations of a complex RF array implementation from its design.

As corroborated by the results of this thesis, building higher density receivers, though challenging, is another avenue for pursuing higher SNR. At the time of this writing, there are efforts in CMRR to build a 128-channel receiver array for human brain imaging at 10.5T. Noise correlation can be more challenging to manage at 128-channels. Moreover, the presence of substantial cabling (and cable traps) in the transmit field can distort transmit homogeneity and efficiency. Such challenges continue to pique the curiosity of MR hardware researchers. By itself, the expected peripheral SNR gain of going to 128 channels alone may not be sufficient to justify the effort to address these challenges if, arguably, other receiver designs could promise similar SNR gains with less channel counts (and by extension, less challenges). Nevertheless, to my knowledge, no alternative receiver design has yet shown any promise of parallel imaging performance on par with a 128-channel 
loop-based receiver, even in simulation.

Beyond loop-based arrays, other receive array designs have also proved promising [91]. A 32-channel sleeve-antenna receiver was shown to provide roughly $15 \%$ more central and $300 \%$ more peripheral SNR compared to the 32-channel loop-based receiver, both at 10.5T, at the expense of a 50\% reduction of the average SNR at the top of the brain. This and other novel receive array designs, each of which has its own advantages and disadvantages in terms of SNR, $g$-factor, and transmit interaction, provide interesting targets for academic investigation.

Ensuring the safety of RF transmitters for in-vivo human studies appears to be a bottleneck in ultra-high field MR hardware research. While not a focus of this thesis, safety validation procedures are poised for consequential innovations. However, these innovations are contingent upon several subproblems being isolated and addressed. The manual construction of RF coils may gradually be automated to minimize inevitable deviations from design, the constraints on the simulated versus experimental magnetic field match can arguably be relaxed in small, partial and calculated steps, or a paradigm shift or breakthrough in the electromagnetic/circuit co-simulation pipeline (e.g. from full-wave Maxwell solvers to statistical or data-driven models, see for example [113], or solving explicitly for parameters of the electromagnetic model that minimize the spatial field mismatch, see for example [114]) may help resolve the existing field mismatches. Given the interdisciplinary history of MR research, some of these advances will probably emerge from progress in tangent fields.

Finally, various conventional parallel imaging reconstruction techniques, including, among others, compressed sensing derivatives, have evolved into and are combined with variations of deep learning methods to improve their performance. While deep learning techniques have made inroads into various MR lines of research, parallel image reconstruction using purely data driven methods seems to be at its infancy. Purely data driven 
reconstruction methods often do not provide explicit analytical guarantee of anatomical fidelity of the reconstructed images. This, of course, has to be resolved before such reconstruction models can be used responsibly and reliably in clinical settings.

More broadly, in deep learning applications in MR, some of the questions that invite further investigation revolve around generalizability: how applicable are models trained on a certain dataset to instances from a different distribution. In MR, this difference can be due to a different scanner or hardware, field strength, acquisition protocol, anatomy of interest, pathological cases, artifacts, etc. Another promising future direction of research is interpretability: medical professionals are interested in transparent decisions because transparency would enable them to understand the decision process and intervene when the deep learning model makes a mistake. Providing tools for interpreting these models is an exciting field of research. 
BIBLIOGRAPHY

[1] K. Uğurbil, E. Auerbach, S. Moeller, et al., "Brain imaging with improved acceleration and SNR at 7 Tesla obtained with 64-channel receive array," Magnetic Resonance in Medicine, vol. 82, no. 1, pp. 495-509, Jul. 2019. [Online]. Available: https://onlinelibrary.wiley.com/ doi/abs/10.1002/mrm.27695.

[2] N. Tavaf, S. Jungst, R. L. Lagore, et al., "A Self-decoupled 64 Channel Receive Array for Human Brain MRI at 10.5T," International Society of Magnetic Resonance in Medicine, p. 2618, 2021.

[3] N. Tavaf, R. L. Lagore, S. Jungst, et al., "A Self-Decoupled 32 Channel Receive Array for Human Brain Magnetic Resonance Imaging at 10.5T," arxiv preprint arXiv: 2009.07163v2, Sep. 2020. [Online]. Available: http://arxiv.org/abs/2009.07163.

[4] W. Durant, Our Oriental Heritage (Story of Civilization). New York: Simon \& Schuster, 1954, p. 1047.

[5] M. E. Ladd, P. Bachert, M. Meyerspeer, et al., "Pros and cons of ultra-high-field MRI/MRS for human application," Progress in Nuclear Magnetic Resonance Spectroscopy, vol. 109, pp. 1-50, Dec. 2018. [Online]. Available: https://www.sciencedirect.com/science/article/ pii/S007965651830013X.

[6] K. Uğurbil, "Imaging at ultrahigh magnetic fields: History, challenges, and solutions," NeuroImage, vol. 168, pp. 7-32, Mar. 2018. [Online]. Available: https://www.sciencedirect. com/science/article/pii/S105381191730575X.

[7] K. Ugurbil, "Magnetic Resonance Imaging at Ultrahigh Fields," IEEE Transactions on Biomedical Engineering, vol. 61, no. 5, pp. 1364-1379, May 2014. [Online]. Available: http://ieeexplore.ieee.org/document/6778087/. 
[8] S. O. Dumoulin, A. Fracasso, W. van der Zwaag, et al., "Ultra-high field MRI: Advancing systems neuroscience towards mesoscopic human brain function," NeuroImage, vol. 168, pp. 345-357, Mar. 2018. [Online]. Available: https ://www. sciencedirect.com/science/ article/pii/S1053811917300289?via\%3Dihub.

[9] R. Boyacioğlu, J. Schulz, N. C. Müller, et al., "Whole brain, high resolution multiband spinecho EPI fMRI at 7 T: A comparison with gradient-echo EPI using a color-word Stroop task," NeuroImage, vol. 97, pp. 142-150, Aug. 2014. [Online]. Available: https://www. sciencedirect.com/science/article/pii/S1053811914002663?via\%3Dihub.

[10] K. Uludağ and P. Blinder, "Linking brain vascular physiology to hemodynamic response in ultra-high field MRI," NeuroImage, vol. 168, pp. 279-295, Mar. 2018. [Online]. Available: https://www.sciencedirect.com/science/article/pii/S1053811917301751.

[11] F. De Martino, E. Yacoub, V. Kemper, et al., "The impact of ultra-high field MRI on cognitive and computational neuroimaging," NeuroImage, vol. 168, no. March, pp. 366-382, Mar. 2018. [Online]. Available: http://dx.doi.org/10.1016/j.neuroimage.2017.03.060.

[12] X. Wu, E. J. Auerbach, A. T. Vu, et al., "High-resolution whole-brain diffusion MRI at 7T using radiofrequency parallel transmission," Magnetic Resonance in Medicine, vol. 80, no. 5, pp. 1857-1870, Nov. 2018. [Online]. Available: http://doi.wiley.com/10.1002/mrm. 27189.

[13] K. Uğurbil, J. Xu, E. J. Auerbach, et al., "Pushing spatial and temporal resolution for functional and diffusion MRI in the Human Connectome Project," NeuroImage, vol. 80, pp. 80104, Oct. 2013. [Online]. Available: https://www.sciencedirect.com/science/article/pii/ S1053811913005065?via\%3Dihub.

[14] D. Gallichan, "Diffusion MRI of the human brain at ultra-high field (UHF): A review," NeuroImage, vol. 168, pp. 172-180, Mar. 2018. [Online]. Available: https://www.sciencedirect. com/science/article/pii/S1053811917303300.

[15] A. Vu, E. Auerbach, C. Lenglet, et al., "High resolution whole brain diffusion imaging at $7 \mathrm{~T}$ for the Human Connectome Project," NeuroImage, vol. 122, pp. 318-331, Nov. 2015. [Online]. Available: https ://www . sciencedirect . com/science/article / pii / S1053811915007120?via\%3Dihub.

[16] E. C. Obusez, M. Lowe, S.-H. Oh, et al., "7T MR of intracranial pathology: Preliminary observations and comparisons to 3T and 1.5T," NeuroImage, vol. 168, pp. 459-476, Mar. 2018. [Online]. Available: https : / / www . sciencedirect . com / science / article / pii / S1053811916306486?via\%3Dihub. 
[17] P. Sati, "Diagnosis of multiple sclerosis through the lens of ultra-high-field MRI," Journal of Magnetic Resonance, vol. 291, pp. 101-109, Jun. 2018. [Online]. Available: https:// www.sciencedirect.com/science/article/pii/S1090780718300454.

[18] S. Trattnig, E. Springer, W. Bogner, et al., "Key clinical benefits of neuroimaging at 7T," NeuroImage, vol. 168, no. November, pp. 477-489, Mar. 2016. [Online]. Available: https: //www.sciencedirect.com/science/article/pii/S1053811916306516?via\%3Dihub.

[19] J. H. Duyn, "Studying brain microstructure with magnetic susceptibility contrast at highfield," NeuroImage, vol. 168, pp. 152-161, Mar. 2018. [Online]. Available: https://www. sciencedirect.com/science/article/pii/S105381191730157X?via\%3Dihub.

[20] R. W. Brown, Y.-c. N. Cheng, E. M. Haacke, et al., Magnetic Resonance Imaging, 2nd. John Wiley \& Sons Ltd, 2014, p. 978. [Online]. Available: http://doi.wiley.com/10.1002/ 9781118633953.

[21] P. Mansfield, A. A. Maudsley, and B. Sc, "Medical imaging by NMR," British Journal of Radiology, vol. 50, pp. 188-194, 1977.

[22] P. C. Lauterbur, "Image formation by induced local interactions: Examples employing nuclear magnetic resonance," Nature, vol. 242, no. 5394, pp. 190-191, 1973. [Online]. Available: https://www.nature.com/articles/242190a0.

[23] R. Pohmann, O. Speck, and K. Scheffler, "Signal-to-noise ratio and MR tissue parameters in human brain imaging at 3, 7, and 9.4 tesla using current receive coil arrays," Magnetic Resonance in Medicine, vol. 75, no. 2, pp. 801-809, Feb. 2016. [Online]. Available: http: //doi.wiley.com/10.1002/mrm.25677.

[24] D. B. Twieg, "The k-trajectory formulation of the NMR imaging process with applications in analysis and synthesis of imaging methods," Medical Physics, vol. 10, no. 5, pp. 610621, Sep. 1983. [Online]. Available: http://doi.wiley.com/10.1118/1.595331.

[25] P. B. Roemer, W. A. Edelstein, C. E. Hayes, et al., "The NMR phased array," Magnetic Resonance in Medicine, vol. 16, no. 2, pp. 192-225, Nov. 1990. [Online]. Available: http: //doi.wiley.com/10.1002/mrm.1910160203.

[26] G. C. Wiggins, J. R. Polimeni, A. Potthast, et al., "96-Channel receive-only head coil for 3 Tesla: Design optimization and evaluation," Magnetic Resonance in Medicine, vol. 62, no. 3, pp. 754-762, Sep. 2009. [Online]. Available: http://doi.wiley.com/10.1002/mrm. 22028.

[27] F. Wiesinger, P. Boesiger, and K. P. Pruessmann, "Electrodynamics and ultimate SNR in parallel MR imaging," Magnetic Resonance in Medicine, vol. 52, no. 2, pp. 376-390, Aug. 2004. [Online]. Available: http://doi.wiley.com/10.1002/mrm.20183. 
[28] F. Wiesinger, N. De Zanche, and K. P. Pruessmann, "Approaching Ultimate SNR with Finite Coil Arrays," Proceedings of the International Society for Magnetic Resonance in Medicine 13, vol. 13, no. 1, p. 672, 2005. [Online]. Available: http://cds.ismrm.org/ismrm2005/Files/00672.pdf.

[29] F. Wiesinger, P.-F. Van de Moortele, G. Adriany, et al., "Potential and feasibility of parallel MRI at high field," NMR in Biomedicine, vol. 19, no. 3, pp. 368-378, May 2006. [Online]. Available: http://doi.wiley.com/10.1002/nbm.1050.

[30] M. V. Vaidya, D. K. Sodickson, and R. Lattanzi, "Approaching ultimate intrinsic SNR in a uniform spherical sample with finite arrays of loop coils," Concepts in Magnetic Resonance Part B: Magnetic Resonance Engineering, vol. 44, no. 3, pp. 53-65, Aug. 2014. [Online]. Available: http://doi.wiley.com/10.1002/cmr.b.21268.

[31] R. Lattanzi, G. C. Wiggins, B. Zhang, et al., "Approaching ultimate intrinsic signal-to-noise ratio with loop and dipole antennas," Magnetic Resonance in Medicine, vol. 79, no. 3, pp. 1789-1803, Mar. 2018. [Online]. Available: https://www.ncbi.nlm.nih.gov/pmc/ articles/PMC5754268/.

[32] B. Keil and L. L. Wald, "Massively parallel MRI detector arrays," Journal of Magnetic Resonance, vol. 229, pp. 75-89, Apr. 2013. [Online]. Available: https://linkinghub.elsevier. com/retrieve/pii/S109078071300030X.

[33] M. Blaimer, F. Breuer, M. Mueller, et al., "SMASH, SENSE, PILS, GRAPPA," Topics in Magnetic Resonance Imaging, vol. 15, no. 4, pp. 223-236, Aug. 2004. [Online]. Available: http://journals.lww.com/00002142-200408000-00002.

[34] D. K. Sodickson and W. J. Manning, "Simultaneous acquisition of spatial harmonics (SMASH): Fast imaging with radiofrequency coil arrays," Magnetic Resonance in Medicine, vol. 38, no. 4, pp. 591-603, Oct. 1997. [Online]. Available: http://doi.wiley. com/10.1002/mrm.1910380414.

[35] K. P. Pruessmann, M. Weiger, M. B. Scheidegger, et al., "SENSE: Sensitivity encoding for fast MRI," Magnetic Resonance in Medicine, vol. 42, no. 5, pp. 952-962, Nov. 1999.

[36] M. A. Griswold, P. M. Jakob, R. M. Heidemann, et al., "Generalized autocalibrating partially parallel acquisitions (GRAPPA)," Magnetic Resonance in Medicine, vol. 47, no. 6, pp. 1202-1210, Jun. 2002. [Online]. Available: http://doi.wiley.com/10.1002/mrm.10171.

[37] F. Knoll, J. Zbontar, A. Sriram, et al., "fastMRI: A Publicly Available Raw k-Space and DICOM Dataset of Knee Images for Accelerated MR Image Reconstruction Using Machine Learning," Radiology: Artificial Intelligence, vol. 2, no. 1, e190007, Jan. 2020. [Online]. Available: https://pubs.rsna.org/doi/abs/10.1148/ryai.2020190007. 
[38] J. Zbontar, F. Knoll, A. Sriram, et al., "fastMRI: An Open Dataset and Benchmarks for Accelerated MRI,” Nov. 2018. [Online]. Available: http://arxiv.org/abs/1811.08839.

[39] F. A. Breuer, S. A. Kannengiesser, M. Blaimer, et al., "General formulation for quantitative G-factor calculation in GRAPPA reconstructions," Magnetic Resonance in Medicine, vol. 62, no. 3, pp. 739-746, Sep. 2009. [Online]. Available: http://doi.wiley.com/10.1002/ mrm.22066.

[40] F. Wiesinger, P.-F. Van de Moortele, G. Adriany, et al., "Parallel imaging performance as a function of field strength - An experimental investigation using electrodynamic scaling," Magnetic Resonance in Medicine, vol. 52, no. 5, pp. 953-964, Nov. 2004. [Online]. Available: http://doi.wiley.com/10.1002/mrm.20281.

[41] O. Ocali and E. Atalar, "Ultimate intrinsic signal-to-noise ratio in MRI," Magnetic Resonance in Medicine, vol. 39, no. 3, pp. 462-473, Mar. 1998. [Online]. Available: http: //doi.wiley.com/10.1002/mrm.1910390317.

[42] D. Donoho, "Compressed sensing," IEEE Transactions on Information Theory, vol. 52, no. 4, pp. 1289-1306, Apr. 2006. [Online]. Available: http://ieeexplore.ieee.org/document/ $1614066 /$.

[43] D. Liang, J. Cheng, Z. Ke, et al., "Deep Magnetic Resonance Image Reconstruction: Inverse Problems Meet Neural Networks," IEEE Signal Processing Magazine, vol. 37, no. 1, pp. 141-151, Jan. 2020.

[44] I. J. Goodfellow, J. Pouget-Abadie, M. Mirza, et al., "Generative Adversarial Networks," Jun. 2014. [Online]. Available: http://www. github.com/goodfeli/adversarial \% 20http: //arxiv.org/abs/1406.2661.

[45] M. Mirza and S. Osindero, "Conditional Generative Adversarial Nets," Nov. 2014. [Online]. Available: http://arxiv.org/abs/1411.1784.

[46] X. Yan, J. C. Gore, and W. A. Grissom, "Self-decoupled radiofrequency coils for magnetic resonance imaging," Nature Communications, vol. 9, no. 1, p. 3481, Dec. 2018. [Online]. Available: http://www.nature.com/articles/s41467-018-05585-8.

[47] S. Aja-Fernández, G. Vegas-Sánchez-Ferrero, and A. Tristán-Vega, "Noise estimation in parallel MRI: GRAPPA and SENSE," Magnetic Resonance Imaging, vol. 32, no. 3, pp. 281-290, Apr. 2014. [Online]. Available: https://linkinghub.elsevier.com/retrieve/pii/ S0730725X13003810.

[48] C. Findeklee, "Array Noise Matching-Generalization, Proof and Analogy to Power Matching," IEEE Transactions on Antennas and Propagation, vol. 59, no. 2, pp. 452-459, Feb. 2011. [Online]. Available: http://ieeexplore.ieee.org/document/5654544/. 
[49] G. Shajan, M. Kozlov, J. Hoffmann, et al., "A 16-channel dual-row transmit array in combination with a 31-element receive array for human brain imaging at 9.4 T," Magnetic Resonance in Medicine, vol. 71, no. 2, pp. 870-879, Feb. 2014. [Online]. Available: http: //doi.wiley.com/10.1002/mrm.24726.

[50] G. Shajan, J. Hoffmann, G. Adriany, et al., "A 7T Head Coil with 16-channel dual-row transmit and 31-channel receive for pTx applications," in 24th Annual Meeting and Exhibition of the International Society for Magnetic Resonance in Medicine, 2016. [Online]. Available: http://archive.ismrm.org/2016/2132.html.

[51] B. Keil, J. N. Blau, S. Biber, et al., "A 64-channel 3T array coil for accelerated brain MRI," Magnetic Resonance in Medicine, vol. 70, no. 1, pp. 248-258, Jul. 2013. [Online]. Available: http://doi.wiley.com/10.1002/mrm.24427.

[52] N. Tavaf, R. L. Lagore, S. Moen, et al., "A 15-channel loop dipole array for in-vivo swine head MR imaging at 10.5T," in International Society of Magnetic Resonance in Medicine, 2019, p. 1445. [Online]. Available: https://index.mirasmart.com/ISMRM2019/PDFfiles/ 1445.html.

[53] K. Lakshmanan, M. Cloos, R. Lattanzi, et al., "The Loopole Antenna: Capturing Magnetic and Electric Dipole Fields with a Single Structure to Improve Transmit and Receive Performance," Proc. Intl. Soc. Mag. Reson. Med. 22, p. 0397, 2014. [Online]. Available: https://cds.ismrm.org/protected/14MProceedings/files/0397.pdf.

[54] B. Keil, "Construction of Receive Arrays," Proc. Intl. Soc. Mag. Reson. Med., vol. 21, pp. 1-10, 2013.

[55] N. Tavaf, R. L. Lagore, S. Jungst, et al., "Developing High Channel Count Receive Arrays for Human Brain Imaging at 10.5T," International Society of Magnetic Resonance in Medicine, p. 4026, 2020. [Online]. Available: http://archive.ismrm.org/2020/4026.html.

[56] R. F. Lee, R. O. Giaquinto, and C. J. Hardy, "Coupling and decoupling theory and its application to the MRI phased array," Magnetic Resonance in Medicine, vol. 48, no. 1, pp. 203-213, Jul. 2002. [Online]. Available: http://doi.wiley.com/10.1002/mrm.10186.

[57] X. Zhang and A. Webb, "Design of a capacitively decoupled transmit/receive NMR phased array for high field microscopy at 14.1 T," Journal of Magnetic Resonance, vol. 170, no. 1, pp. 149-155, Sep. 2004.

[58] R. G. Pinkerton, E. A. Barberi, and R. S. Menon, "Noise properties of a NMR transceiver coil array," Journal of Magnetic Resonance, vol. 171, no. 1, pp. 151-156, Nov. 2004. 
[59] N. I. Avdievich, J. W. Pan, and H. P. Hetherington, "Resonant inductive decoupling (RID) for transceiver arrays to compensate for both reactive and resistive components of the mutual impedance," NMR in Biomedicine, vol. 26, no. 11, pp. 1547-1554, Nov. 2013. [Online]. Available: http://doi.wiley.com/10.1002/nbm.2989.

[60] C. Findeklee, O. Lips, P. Vernickel, et al., "Preamp decoupling improves SNR and the earch is flat," Proc. Intl. Soc. Mag. Reson. Med, vol. 27, p. 0563, 2019.

[61] P. Hasgall, F. Di Gennaro, C. Baumgartner, et al., IT'IS Database for thermal and electromagnetic parameters of biological tissues, 2018. [Online]. Available: itis.swiss/database.

[62] P. M. Robson, A. K. Grant, A. J. Madhuranthakam, et al., "Comprehensive quantification of signal-to-noise ratio and $\mathrm{g}$-factor for image-based and $\mathrm{k}$-space-based parallel imaging reconstructions," Magnetic Resonance in Medicine, vol. 60, no. 4, pp. 895-907, Oct. 2008. [Online]. Available: http://doi.wiley.com/10.1002/mrm.21728.

[63] P. Kellman and E. R. McVeigh, "Image reconstruction in SNR units: A general method for SNR measurement," Magnetic Resonance in Medicine, vol. 54, no. 6, pp. 1439-1447, Dec. 2005. [Online]. Available: http://doi.wiley.com/10.1002/mrm.20713.

[64] J. Wang, "Relation between noise correlation and transmission coefficient of surface coils for magnetic resonance imaging," in Proceedings of 17th International Conference of the Engineering in Medicine and Biology Society, vol. 1, IEEE, 1993, pp. 469-470. [Online]. Available: http://ieeexplore.ieee.org/document/575204/.

[65] B. Guérin, J. F. Villena, A. G. Polimeridis, et al., "The ultimate signal-to-noise ratio in realistic body models," Magnetic Resonance in Medicine, vol. 78, no. 5, pp. 1969-1980, Nov. 2017. [Online]. Available: http://doi.wiley.com/10.1002/mrm.26564.

[66] W. Schnell, W. Renz, M. Vester, et al., "Ultimate signal-to-noise-ratio of surface and body antennas for magnetic resonance imaging," IEEE Transactions on Antennas and Propagation, vol. 48, no. 3, pp. 418-428, Mar. 2000. [Online]. Available: http://ieeexplore.ieee.org/ document/841903/.

[67] S. Schmitter, S. Schnell, K. Uğurbil, et al., "Towards high-resolution 4D flow MRI in the human aorta using kt-GRAPPA and B1+ shimming at 7T," Journal of Magnetic Resonance Imaging, vol. 44, no. 2, pp. 486-499, 2016.

[68] M. Arcan, E. Urk, X. Wu, et al., "Toward Imaging the Body at 10.5 Tesla," Magn Reson Med, vol. 77, pp. 434-443, 2017. [Online]. Available: https://onlinelibrary.wiley.com/doi/ pdf/10.1002/mrm.26487.

[69] T. M. Fiedler, M. E. Ladd, and A. K. Bitz, "SAR Simulations \& Safety," NeuroImage, no. March, 2017. 
[70] Ö. Ipek, "Radio-frequency coils for ultra-high field magnetic resonance," Analytical Biochemistry, vol. 529, pp. 10-16, Jul. 2017. [Online]. Available: http://linkinghub.elsevier. com/retrieve/pii/S000326971730146X.

[71] P.-F. F. Van de Moortele, C. Akgun, G. Adriany, et al., "B1 destructive interferences and spatial phase patterns at $7 \mathrm{~T}$ with a head transceiver array coil," Magnetic Resonance in Medicine, vol. 54, no. 6, pp. 1503-1518, Dec. 2005. [Online]. Available: http://doi.wiley. com/10.1002/mrm.20708.

[72] J. B. Ra and C. Y. Rim, "Fast imaging using subencoding data sets from multiple detectors," Magnetic Resonance in Medicine, vol. 30, no. 1, pp. 142-145, Jul. 1993. [Online]. Available: http://doi.wiley.com/10.1002/mrm.1910300123.

[73] M. A. Ohliger, A. K. Grant, and D. K. Sodickson, "Ultimate intrinsic signal-to-noise ratio for parallel MRI: Electromagnetic field considerations," Magnetic Resonance in Medicine, vol. 50, no. 5, pp. 1018-1030, Nov. 2003. [Online]. Available: http://doi.wiley.com/10. 1002/mrm.10597.

[74] G. Adriany, J. Radder, N. Tavaf, et al., "Evaluation of a 16-Channel Transmitter for Head Imaging at 10.5T," in 2019 International Conference on Electromagnetics in Advanced Applications (ICEAA), IEEE, Sep. 2019, pp. 1171-1174. [Online]. Available: https :// ieeexplore.ieee.org/document/8879131/.

[75] G. Adriany, A. Gozubuyuk, E. Auerbach, et al., "A 32 channel transmit/receive transmission line head array for 3D RF shimming," Proceedings of the joint annual meeting ISMRM-ESMRMB, Berlin, Germany, vol. 15, p. 166, 2007.

[76] G. Adriany, J. Ritter, T. Vaughan, et al., "Experimental verification of enhanced B1 Shim performance with a Z-encoding RF coil array at 7 tesla," Proc. Intl. Soc. Mag. Reson. Med., vol. 3831, 2010.

[77] X. Wu, J. Tian, S. Schmitter, et al., "Distributing coil elements in three dimensions enhances parallel transmission multiband RF performance: A simulation study in the human brain at 7 Tesla," Magnetic Resonance in Medicine, vol. 75, no. 6, pp. 2464-2472, Jun. 2016. [Online]. Available: http://doi.wiley.com/10.1002/mrm.26194.

[78] X. Wu, X. Zhang, J. Tian, et al., "Comparison of RF body coils for MRI at 3T: A simulation study using parallel transmission on various anatomical targets," NMR in Biomedicine, vol. 28, no. 10, pp. 1332-1344, 2015. 
[79] B. Guérin, K. Setsompop, H. Ye, et al., "Design of parallel transmission pulses for simultaneous multislice with explicit control for peak power and local specific absorption rate," Magnetic Resonance in Medicine, vol. 73, no. 5, pp. 1946-1953, May 2015. [Online]. Available: http://doi.wiley.com/10.1002/mrm.25325.

[80] C. Oezerdem, L. Winter, A. Graessl, et al., "16-channel bow tie antenna transceiver array for cardiac MR at 7.0 tesla," Magnetic Resonance in Medicine, vol. 75, no. 6, pp. 25532565, Jun. 2016. [Online]. Available: https://pubmed.ncbi.nlm.nih.gov/26183320/.

[81] D. Seeber, J. Jevtic, and A. Menon, "Floating shield current suppression trap," Concepts in Magnetic Resonance, vol. 21B, no. 1, pp. 26-31, Apr. 2004. [Online]. Available: http: //doi.wiley.com/10.1002/cmr.b.20008.

[82] J. Brown and B. Whitlock, "Common-Mode to Differential-Mode Conver-sion in Shielded Twisted-Pair Cables (Shield-Current-Induced Noise)," 114th AES Convention in Amsterdam, p. 5747, 2003. [Online]. Available: http://www.aes.org/e-lib/.

[83] P. Van de Moortele, C. Snyder, L. DelaBarre, et al., "Shimming at Very High Field with Multiple Element RF Coils: Calibration Tools from Ultra Fast Local Relative B1+/- Phase to Absolute Magnitude B1+ Mapping," in International Society of Magnetic Resonance in Medicine, 2007, p. 1676.

[84] G. Adriany, P.-F. F. Van De Moortele, F. Wiesinger, et al., "Transmit and receive transmission line arrays for 7 tesla parallel imaging," Magnetic Resonance in Medicine, vol. 53, no. 2, pp. 434-445, Feb. 2005. [Online]. Available: http://doi.wiley.com/10.1002/mrm. 20321.

[85] V. L. Yarnykh, “Actual flip-angle imaging in the pulsed steady state: A method for rapid three-dimensional mapping of the transmitted radiofrequency field," Magnetic Resonance in Medicine, vol. 57, no. 1, pp. 192-200, Jan. 2007. [Online]. Available: http://doi.wiley. com/10.1002/mrm.21120.

[86] P. F. Van de Moortele, E. J. Auerbach, C. Olman, et al., "T1 weighted brain images at 7 Tesla unbiased for Proton Density, T2* contrast and RF coil receive B1 sensitivity with simultaneous vessel visualization," NeuroImage, vol. 46, no. 2, pp. 432-446, 2009.

[87] NEMA, "Determination of Signal-to-Noise Ratio (SNR) in Diagnostic Magnetic Resonance Imaging," in NEMA Standards Publication MS 1-2008 (R2014), 2008. [Online]. Available: www.nema.org.

[88] J. Ellermann, U. Goerke, P. Morgan, et al., "Simultaneous bilateral hip joint imaging at 7 Tesla using fast transmit B1 shimming methods and multichannel transmission - a feasibility study," NMR in Biomedicine, vol. 25, no. 10, pp. 1202-1208, 2012. 
[89] A. Pfrommer and A. Henning, "The ultimate intrinsic signal-to-noise ratio of loopand dipole-like current patterns in a realistic human head model," Magnetic Resonance in Medicine, vol. 80, no. 5, pp. 2122-2138, Nov. 2018. [Online]. Available: https :// onlinelibrary.wiley.com/doi/abs/10.1002/mrm.27169.

[90] J. Radder, M. K. Woo, P. F. Van De Moortele, et al., "Optimization and simulation of a 16-channel loop and dipole array for head MRI applications at 10.5 Tesla," Proceedings of the 2017 19th International Conference on Electromagnetics in Advanced Applications, ICEAA 2017, pp. 1828-1831, 2017.

[91] M. K. Woo, L. DelaBarre, M. Waks, et al., "A Novel High Density 32-channel Sleeve Antenna Receiver Array for the Human Head Imaging at 10.5 T," International Society of Magnetic Resonance in Medicine, p. 236, 2021.

[92] N. Tavaf, A. Torfi, K. Ugurbil, et al., "GRAPPA-GANs for Parallel MRI Reconstruction," arXiv preprint arXiv:2101.03135, Jan. 2021. [Online]. Available: http://arxiv.org/abs/2101. 03135 .

[93] A. Torfi, R. A. Shirvani, Y. Keneshloo, et al., "Natural Language Processing Advancements By Deep Learning: A Survey," arXiv:2003.01200v3, Mar. 2020. [Online]. Available: http: //arxiv.org/abs/2003.01200.

[94] I. Goodfellow, J. Pouget-Abadie, M. Mirza, et al., "Generative adversarial networks," Communications of the ACM, vol. 63, no. 11, pp. 139-144, Oct. 2020. [Online]. Available: http: //www.github.com/goodfeli/adversarial.

[95] G. Yang, S. Yu, H. Dong, et al., "DAGAN: Deep De-Aliasing Generative Adversarial Networks for Fast Compressed Sensing MRI Reconstruction," IEEE Transactions on Medical Imaging, vol. 37, no. 6, pp. 1310-1321, 2018.

[96] B. Murugesan, V. R. S, K. Sarveswaran, et al., "Recon-GLGAN: A Global-Local context based Generative Adversarial Network for MRI Reconstruction,” Aug. 2019. [Online]. Available: http://arxiv.org/abs/1908.09262.

[97] H. Emami, M. Dong, and C. K. Glide-Hurst, "Attention-Guided Generative Adversarial Network to Address Atypical Anatomy in Modality Transfer," Jun. 2020. [Online]. Available: http://arxiv.org/abs/2006.15264.

[98] M. Mardani, E. Gong, J. Y. Cheng, et al., "Deep Generative Adversarial Neural Networks for Compressive Sensing MRI," IEEE Transactions on Medical Imaging, vol. 38, no. 1, pp. 167-179, Jan. 2019. [Online]. Available: https :// ieeexplore . ieee.org / document / $8417964 /$. 
[99] P. Deora, B. Vasudeva, S. Bhattacharya, et al., "Structure Preserving Compressive Sensing MRI Reconstruction using Generative Adversarial Networks,” Oct. 2019. [Online]. Available: http://arxiv.org/abs/1910.06067.

[100] S. U. Dar, M. Yurt, M. Shahdloo, et al., "Prior-guided image reconstruction for accelerated multi-contrast mri via generative adversarial networks," IEEE Journal on Selected Topics in Signal Processing, vol. 14, no. 6, pp. 1072-1087, 2020.

[101] N. Tavaf, K. Ugurbil, and P.-F. Van de Moortele, "Reconstruction of Accelerated MR Acquisitions with Conditional Generative Adversarial Networks," in International Society of Magnetic Resonance in Medicine, 2021, p. 723.

[102] O. Ronneberger, P. Fischer, and T. Brox, "U-Net: Convolutional Networks for Biomedical Image Segmentation," Lecture Notes in Computer Science (including subseries Lecture Notes in Artificial Intelligence and Lecture Notes in Bioinformatics), vol. 9351, pp. 234241, May 2015. [Online]. Available: http://arxiv.org/abs/1505.04597.

[103] S. Ioffe and C. Szegedy, "Batch Normalization: Accelerating Deep Network Training by Reducing Internal Covariate Shift," 32nd International Conference on Machine Learning, ICML 2015, vol. 1, pp. 448-456, Feb. 2015. [Online]. Available: https://arxiv.org/abs/1502. $03167 \mathrm{v} 3$.

[104] C. Szegedy, V. Vanhoucke, S. Ioffe, et al., "Rethinking the Inception Architecture for Computer Vision," in Proceedings of the IEEE Computer Society Conference on Computer Vision and Pattern Recognition, vol. 2016-Decem, IEEE Computer Society, Dec. 2016, pp. 2818-2826. [Online]. Available: https://arxiv.org/abs/1512.00567v3.

[105] O. Russakovsky, J. Deng, H. Su, et al., "ImageNet Large Scale Visual Recognition Challenge," International Journal of Computer Vision, vol. 115, no. 3, pp. 211-252, Sep. 2014. [Online]. Available: http://arxiv.org/abs/1409.0575.

[106] C. Ledig, L. Theis, F. Huszar, et al., "Photo-Realistic Single Image Super-Resolution Using a Generative Adversarial Network," Proceedings - 30th IEEE Conference on Computer Vision and Pattern Recognition, CVPR 2017, vol. 2017-Janua, pp. 105-114, Sep. 2016. [Online]. Available: http://arxiv.org/abs/1609.04802.

[107] Z. Wang, A. C. Bovik, H. R. Sheikh, et al., "Image Quality Assessment: From Error Visibility to Structural Similarity," IEEE Transactions on Image Processing, vol. 13, no. 4, 2004. [Online]. Available: http://www.cns.nyu.edu/ lcv/ssim/.. 
[108] D. P. Kingma and J. L. Ba, "Adam: A method for stochastic optimization," in 3rd International Conference on Learning Representations, ICLR 2015 - Conference Track Proceedings, International Conference on Learning Representations, ICLR, Dec. 2015. [Online]. Available: https://arxiv.org/abs/1412.6980v9.

[109] N. Tavaf, J. Radder, R. L. Lagore, et al., "Design of a Self Decoupled 16 Channel Transmitter for Human Brain MRI at 447MHz," arXiv preprint arXiv:2103.07516, 2021. [Online]. Available: https://arxiv.org/abs/2103.07516.

[110] G. Carluccio, B. Zhang, R. Lattanzi, et al., "Effects of Parallel Imaging Acceleration on SNR Improvement with a High-Permittivity Helmet Shaped Former at Different Field Strengths," International Society of Magnetic Resonance in Medicine, p. 1567, 2019.

[111] B. Zhang, G. Adriany, A. Grant, et al., "Shielding Effects on Signal-to-Noise Ratio at UltraHigh Field MRI," International Society of Magnetic Resonance in Medicine, p. 0752, 2020.

[112] B. Zhang, G. Adriany, L. Delabarre, et al., "Effect of radiofrequency shield diameter on signal-to-noise ratio at ultra-high field MRI," Magnetic Resonance in Medicine, mrm.28670, Jan. 2021. [Online]. Available: https://onlinelibrary.wiley.com/doi/10.1002/ mrm. 28670 .

[113] E. Meliadò, A. Raaijmakers, A. Sbrizzi, et al., "A deep learning method for image-based subject-specific local SAR assessment," Magnetic Resonance in Medicine, mrm.27948, Sep. 2019. [Online]. Available: https://onlinelibrary.wiley.com/doi/abs/10.1002/mrm. 27948.

[114] X. Li, J. W. Pan, N. I. Avdievich, et al., "Electromagnetic simulation of a 16-channel head transceiver at $7 \mathrm{~T}$ using circuit-spatial optimization," Magnetic Resonance in Medicine, no. December 2020, mrm.28672, Feb. 2021. [Online]. Available: https://onlinelibrary. wiley.com/doi/10.1002/mrm.28672. 